Florida International University FIU Digital Commons

4-21-2017

\title{
Robot Control for Remote Ophthalmology and Pediatric Physical Rehabilitation
}

Melissa Morris

Florida International University, mmorr009@fiu.edu

DOI: $10.25148 /$ etd.FIDC001981

Follow this and additional works at: https:// digitalcommons.fiu.edu/etd

Part of the Acoustics, Dynamics, and Controls Commons, Biomechanical Engineering Commons, and the Electro-Mechanical Systems Commons

\section{Recommended Citation}

Morris, Melissa, "Robot Control for Remote Ophthalmology and Pediatric Physical Rehabilitation" (2017). FIU Electronic Theses and Dissertations. 3350.

https://digitalcommons.fiu.edu/etd/3350 


\title{
FLORIDA INTERNATIONAL UNIVERSITY
}

Miami, Florida

\section{ROBOT CONTROL FOR REMOTE OPHTHALMOLOGY \\ AND PEDIATRIC PHYSICAL REHABILITATION}

\author{
A dissertation submitted in partial fulfillment of \\ the requirements for the degree of \\ DOCTOR OF PHILOSOPHY \\ in \\ MECHANICAL ENGINEERING \\ by \\ Melissa Morris
}


To: Interim Dean Ranu Jung,

College of Engineering and Computing

This dissertation, written by Melissa Morris, and entitled Robot Control for Remote Ophthalmology and Pediatric Physical Rehabilitation, having been approved in respect to style and intellectual content, is referred to you for judgment.

We have read this dissertation and recommend that it be approved.

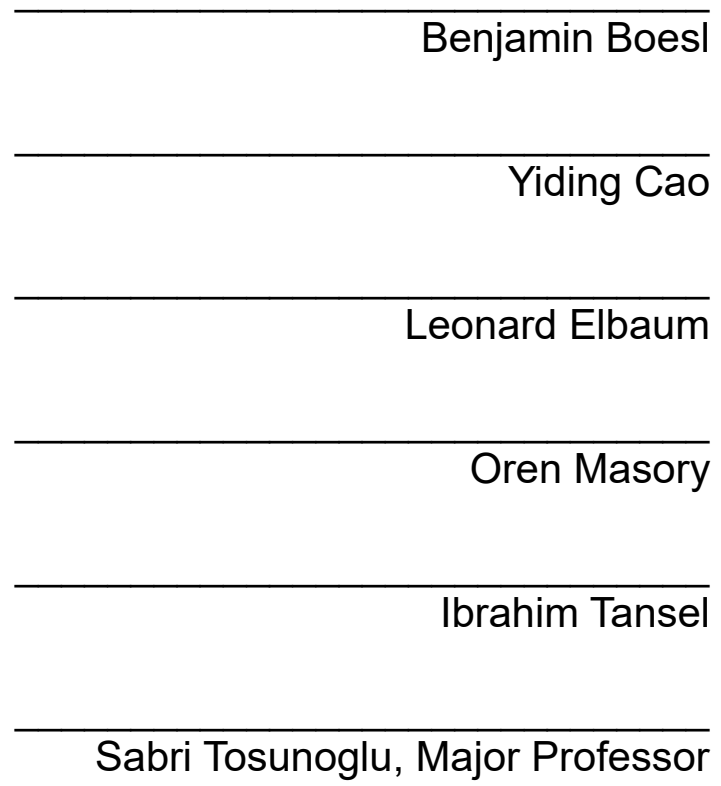

Date of Defense: April 21, 2017

The dissertation of Melissa Morris is approved.

Interim Dean Ranu Jung College of Engineering and Computing

Andres Gil

Vice President for Research and Economic Development and Dean of the University Graduate School

Florida International University, 2017 
(C) Copyright 2017 by Melissa Morris

All rights reserved 


\section{DEDICATION}

To Johnathan, Heather ז"ז, Elizabeth, and Sammy 


\section{ACKNOWLEDGMENTS}

I'd first like to thank my husband, Danny, for his help with some of the construction of the first prototype, particularly cutting the metal brackets and manufacturing some unconventional components.

I would like to thank all of my committee members for sticking through this project even though it didn't follow a simple path through the expected disciplines when I first started. I also appreciate their support when my life didn't go smoothly nor as planned outside of my dissertation as well.

I would like to acknowledge and thank the undergraduate students who participated in various aspects of this work. These include Gabriel Lopez who aided in part of the preliminary study for OphthBot 1; David Larsson, Kassim Kaba, Daniel Irving, Faris Baksh, Shehryar Effendi, Yazeed Almashan, Joshua Dubin, and Bader Alsubaei who performed much of the design and nearly all of the construction of OphthBot 2; and Gilbert Soles, Ricardo Olazo, Angel Mendoza, Jean-Carlo Drada, Raheem Maliki, Khalid Attallah, Dalal Alhaidar, Saleh Alsalem, Luis Ramos, Steven Valencia, Shawnak Verma, and Karina Zornoza who all performed much of the design and all of the construction of some of the physical rehabilitation projects mentioned in this work.

I finally would like to acknowledge the financial support I received while preparing this dissertation. I would like to thank the DOD/Army Research Office for financial support under ARO Grant No. W911NF-11-1-0131 to perform this

research from summer 2014 through spring 2015. I would also like to 
acknowledge Dr. Cesar Levy for the financial support through this grant and also as a teaching assistant in the Department of Mechanical and Materials Engineering for the three years prior. I would also like to thank him for his steadfast mentorship and moral support. The first ophthalmic prototype was completed under a grant provided by Dr. Austin Bach of TYB, LLC in Miami Beach, Florida. He came with the vision, ideas and enthusiasm to get the teleoperated ophthalmic robot prototype underway. Two semesters of the writing phase of the work was completed while under the Dissertation Year Fellowship provided by Florida International University. The financial support is very much appreciated and allowed these projects to happen. 


\author{
ABSTRACT OF THE DISSERTATION \\ ROBOT CONTROL FOR REMOTE OPHTHALMOLOGY \\ AND PEDIATRIC PHYSICAL REHABILITATION
}

by

Melissa Morris

Florida International University, 2017

Miami, Florida

Professor Sabri Tosunoglu, Major Professor

The development of a robotic slit-lamp for remote ophthalmology is the primary purpose of this work. In addition to novel mechanical designs and implementation, it was also a goal to develop a control system that was flexible enough to be adapted with minimal user adjustment to various styles and configurations of slit-lamps. The system was developed with intentions of commercialization, so common hardware was used for all components to minimize the costs. In order to improve performance using this low-cost hardware, investigations were made to attempt to achieve better performance by applying control theory algorithms in the system software. Ultimately, the controller was to be flexible enough to be applied to other areas of human-robot interaction including pediatric rehabilitation via the use of humanoid robotic aids. This application especially requires a robust controller to facilitate safe interaction. Though all of the prototypes were successfully developed and made to work sufficiently with the control hardware, the application of advanced control did not yield notable gains as was hoped. Further investigations were made 
attempting to alter the performance of the control system, but the components selected did not have the physical capabilities for improved response above the original software implemented. Despite this disappointment, numerous novel advances were made in the area of teleoperated ophthalmic technology and pediatric physical rehabilitation tools. This includes a system that is used to remote control a slit-lamp and lens for examinations and some laser procedures. Secondly, a series of of humanoid systems suitable for both medical research and therapeutic modeling were developed. This included a robotic face used as an interactive system for ophthalmic testing and training. It can also be used as one component in an interactive humanoid robotic system that includes hands and arms to allow use of teaching sign language, social skills or modeling occupational therapy tasks. Finally, a humanoid system is presented that can serve as a customized surrogate between a therapist and client to model physical therapy tasks in a realistic manner. These systems are all functional, safe and low-cost to allow for feasible implementation with patients in the near future. 


\section{TABLE OF CONTENTS}

CHAPTER

PAGE

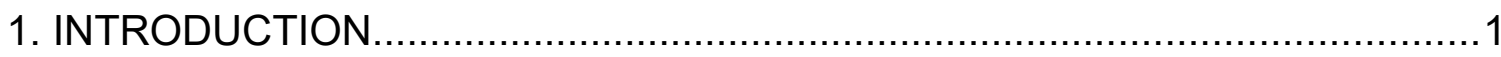

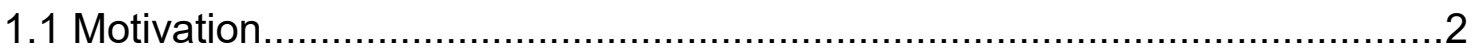

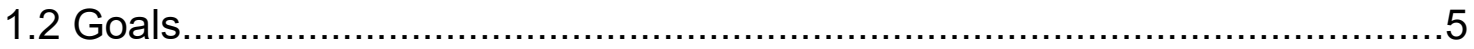

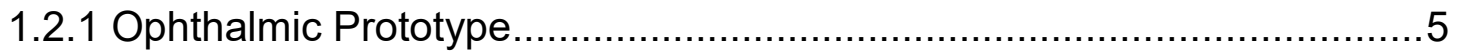

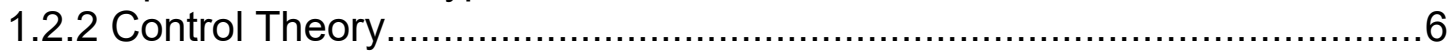

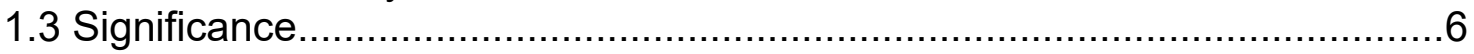

2. ROBOTIC OCULAR SURGERY LITERATURE REVIEW ..........................

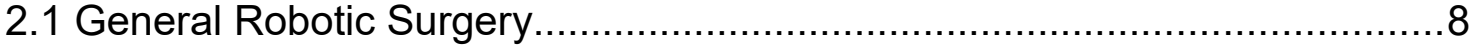

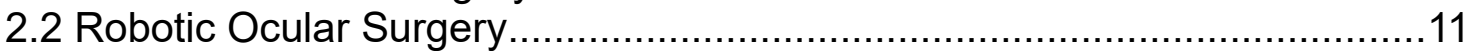

2.2.1 Ophthalmology Basics................................................................11

2.2.1.1 Eye Anatomy and Physiology ...................................................11

2.2.1.2 Examination Procedures and Tools..........................................12

2.2.1.3 Treatment Procedures and Non-Robotic Tools..............................14

2.2.2 Current Ocular Robotic Systems ...................................................16

2.2.3 Current Ocular System Technologies..............................................25

3. PEDIATRIC ROBOTIC THERAPY LITERATURE REVIEW.........................29

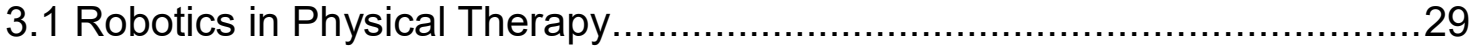

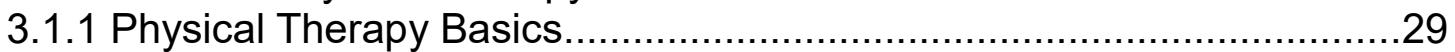

3.1.2 Current Physical Therapy Robotic Systems .......................................33

3.1.3 Current Pediatric Physical Therapy Robotic Systems ...........................36

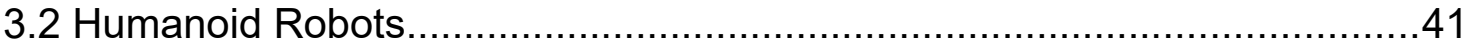

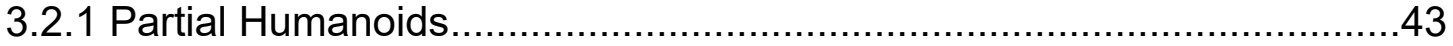

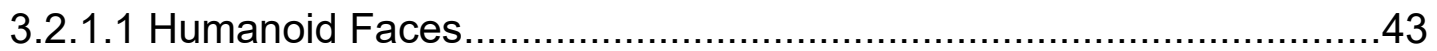

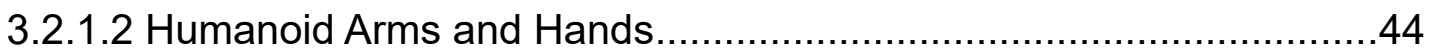

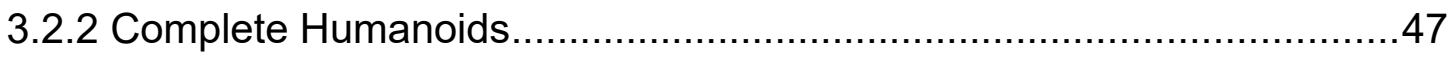

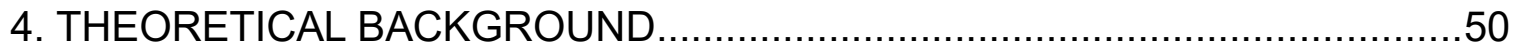

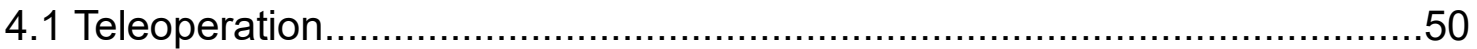

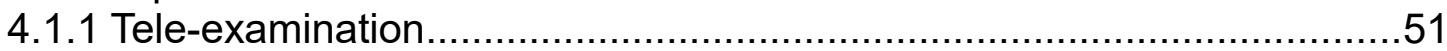

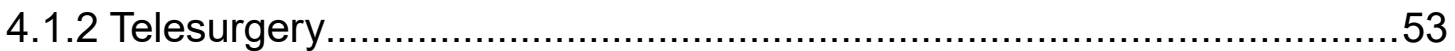

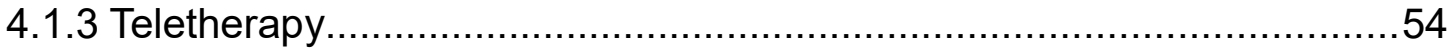

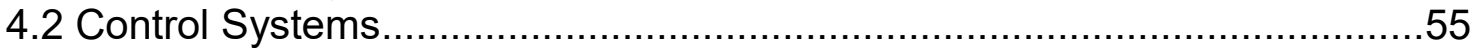

4.3 Control Systems in Medical Robotics....................................................57

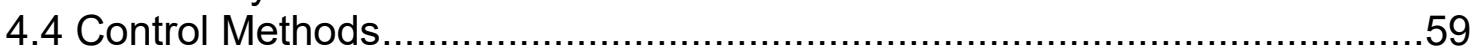

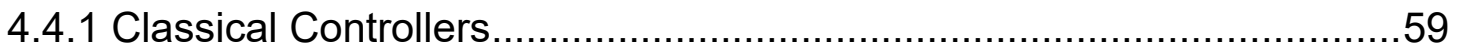

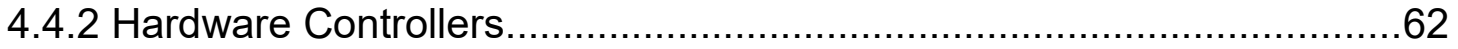

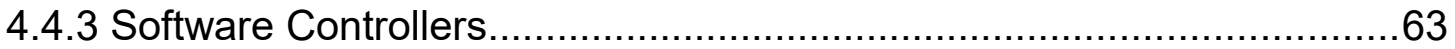

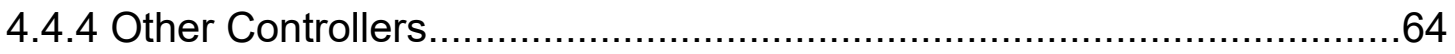


5. OPHTHALMIC PROTOTYPES DESIGN AND CONSTRUCTION..................66

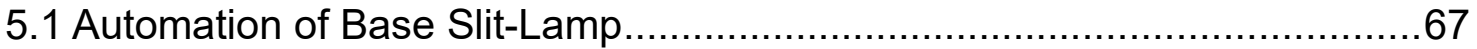

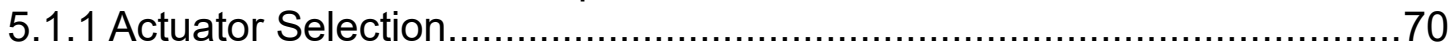

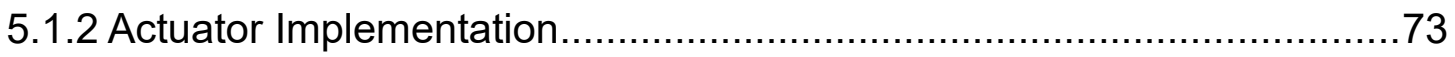

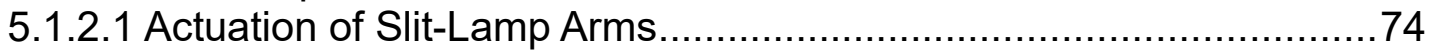

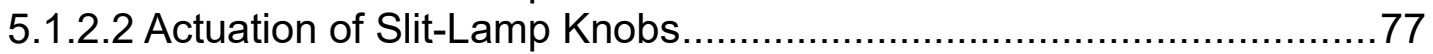

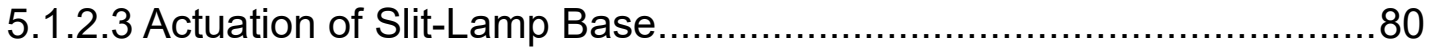

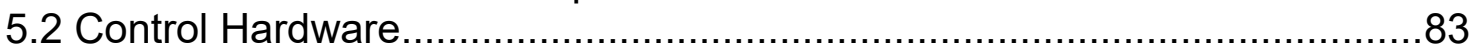

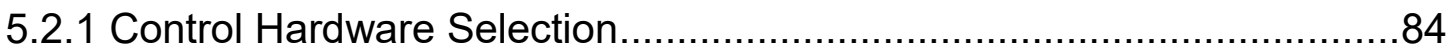

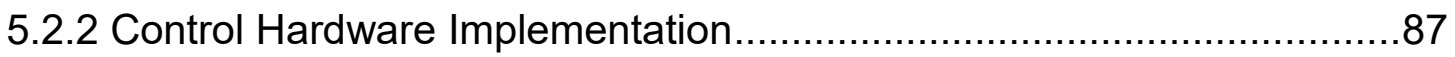

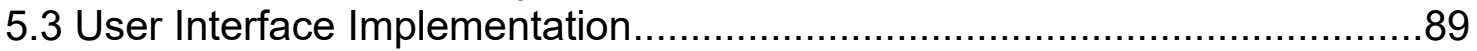

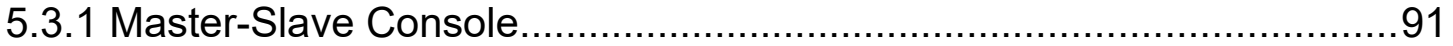

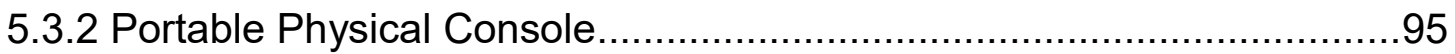

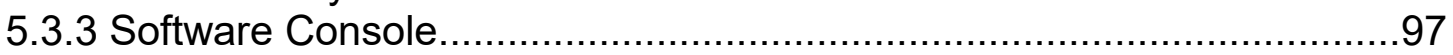

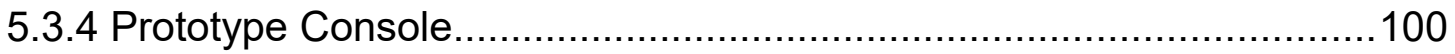

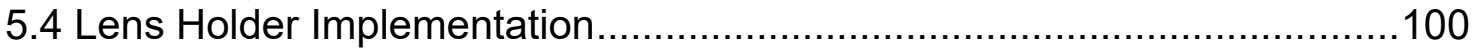

5.4.1 Lens Holder Design Options...................................................102

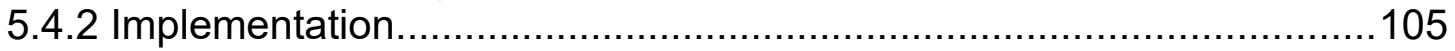

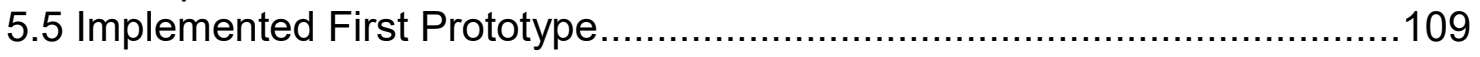

5.6 Second Prototype Design and Construction...........................................110

5.6.1 Automation of the Base Slit-Lamp...............................................110

5.6.2 Lens Holder Implementation......................................................113

5.6.3 Complete OphthBot 2 Implementation...........................................115

6. REHABILITATION ROBOTS DESIGN AND CONSTRUCTION...................117

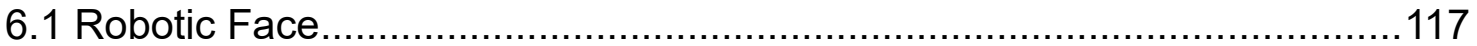

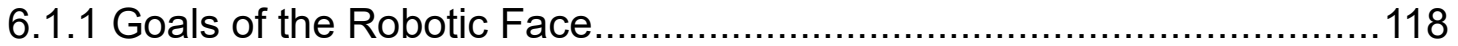

6.1.2 Robotic Face Implementation....................................................119

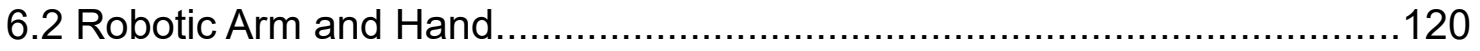

6.2.1 Goals for Robotic Arm and Hand................................................120

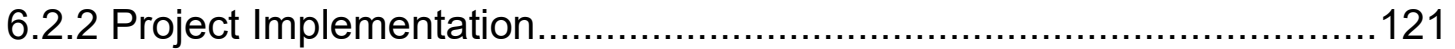

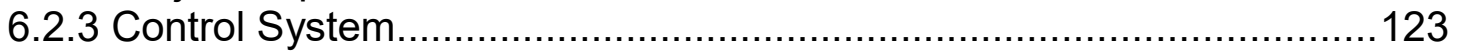

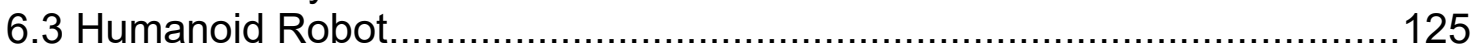

6.3.1 Humanoid Project Goals...........................................................125

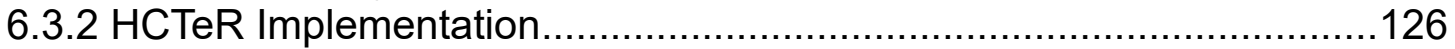

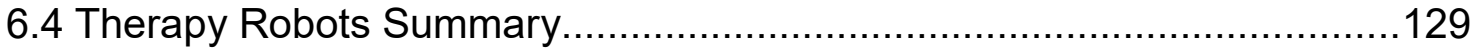

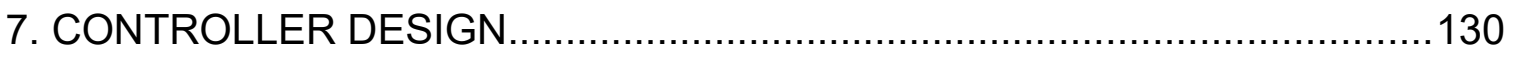

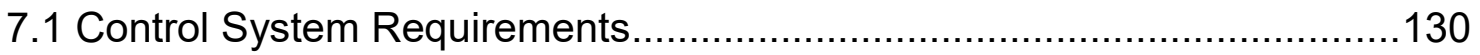

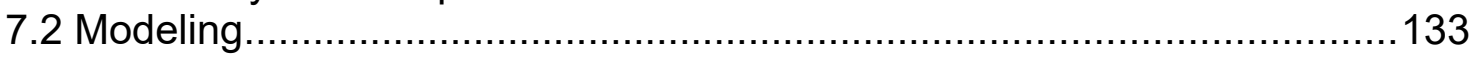

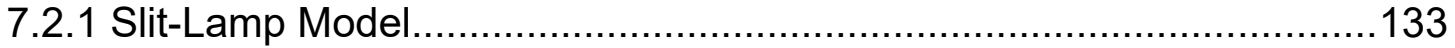

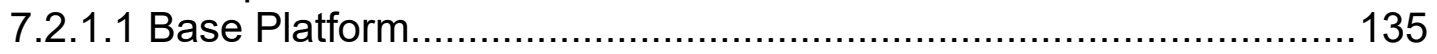

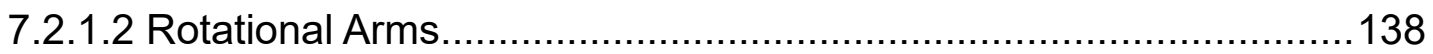

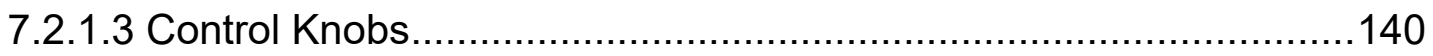

7.2.1.4 Model Limitations.................................................................141 


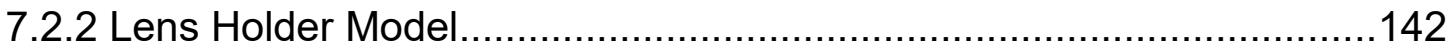

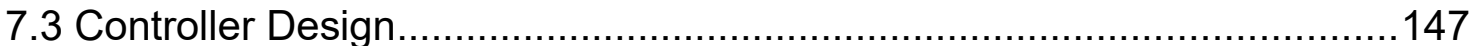

7.4 Controller Implementation.............................................................148

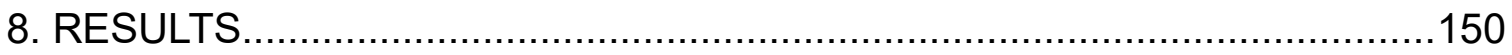

8.1 Functionality of the OphthBot Prototypes...........................................150

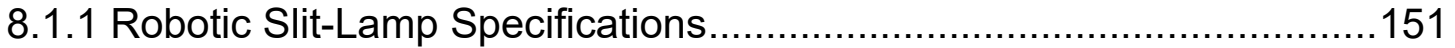

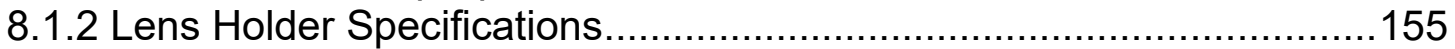

8.2 Comparison of Prototype Performance to Traditional Use.......................157

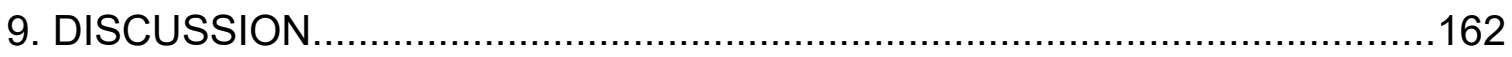

9.1 Prototype Comparisons to Prior Technologies.....................................162

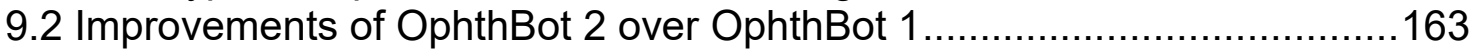

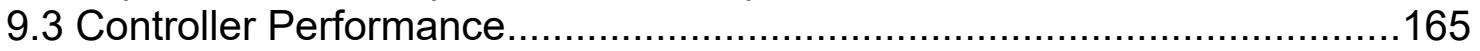

9.4 Experimental Errors and Uncertainty...............................................168

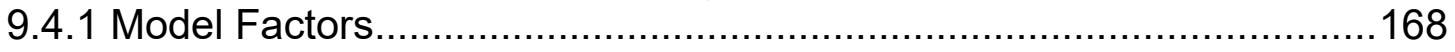

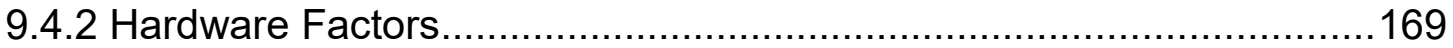

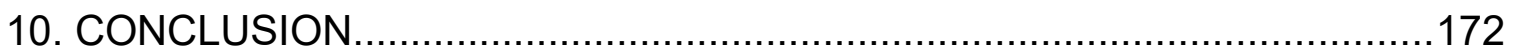

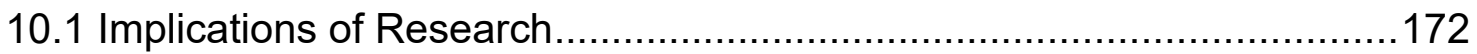

10.2 Future Directions and Recommendations........................................174

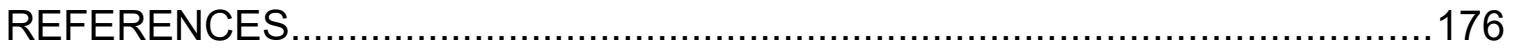

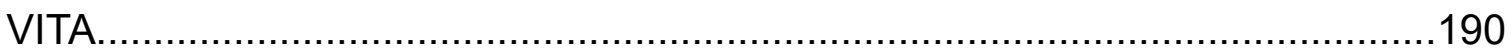




\section{LIST OF FIGURES}

FIGURE

PAGE

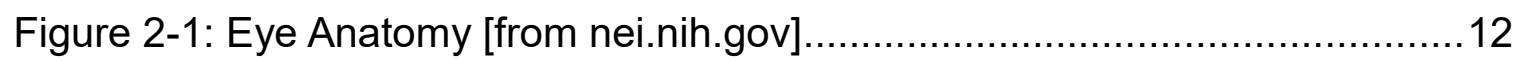

Figure 2-2: "The Eye Robot" [37] ......................................................... 17

Figure 2-3: Experimental ocular ultramicrosurgery robot [122] .......................17

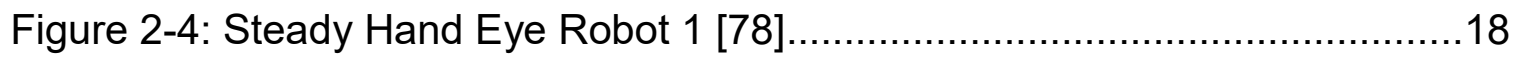

Figure 2-5: Design for a surgical system using a halo positioned by two

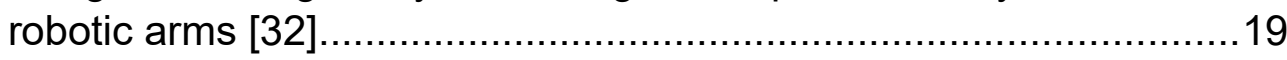

Figure 2-6: A parallel robotic arm for vitreoretinal surgery [83] .........................20

Figure 2-7: Microhand forceps closing under increasing pressure [48].............21

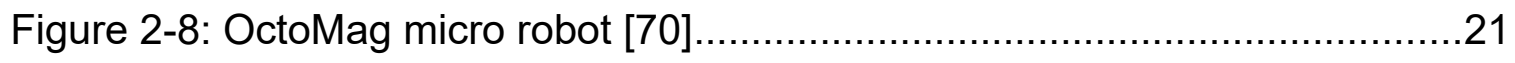

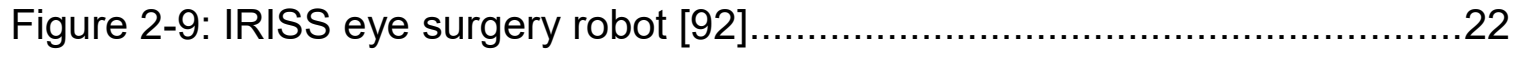

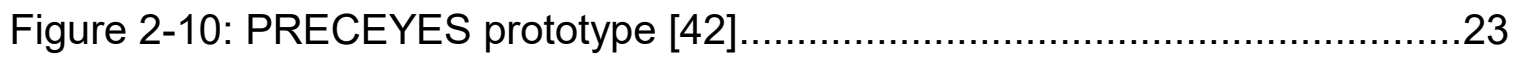

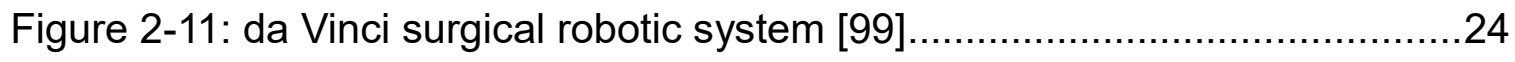

Figure 2-12: Hexapod Surgical System for micro-macro manipulations [10]......25

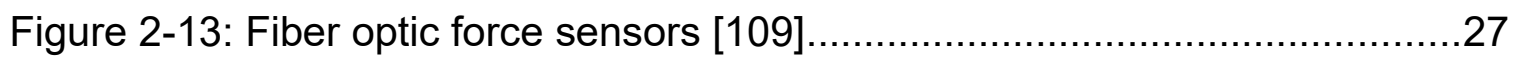

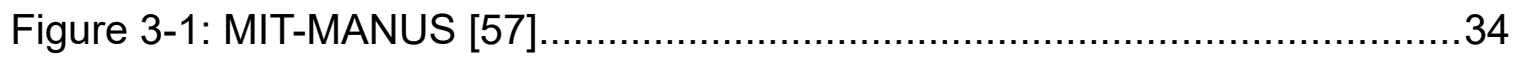

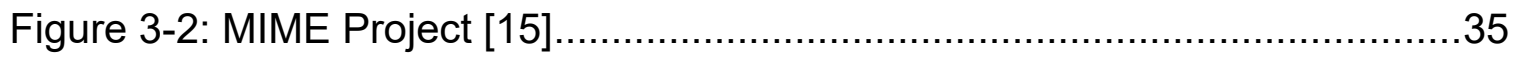

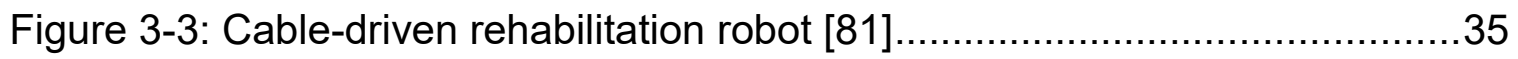

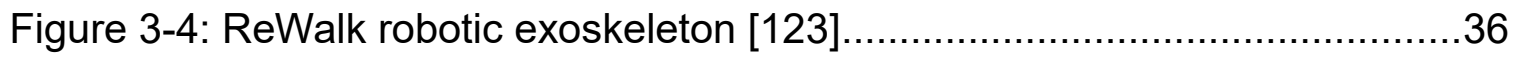

Figure 3-5: InMotion2 system in use with a child [30].....................................38

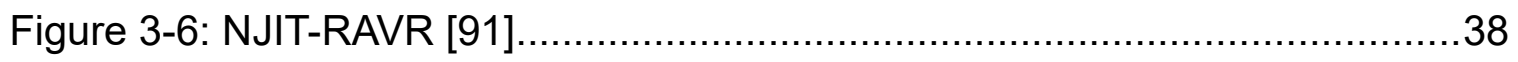

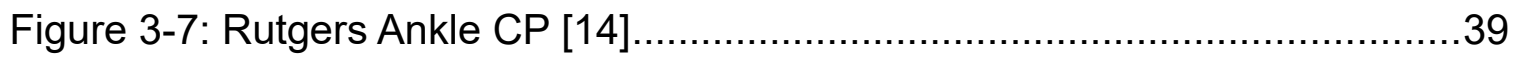

Figure 3-8: DGO Lokomat used for pediatric gait training [75] .........................40

Figure 3-9: Gait Trainer GT I in pediatric use [105] .................................... 41

Figure 3-10: Zeno showing (from left to right) happy, sad and angry faces [100]...... 
Figure 4-1: Robotic slit-lamp for teleophthalmology developed in Thailand [21].....

Figure 4-2: Schematic of a simple hysteresis control system [3].....................60

Figure 4-3: Schematic of a basic PID controller..............................................

Figure 5-1: Major system components of OphthBot.......................................67

Figure 5-2: Base slit-lamp used for the prototype ..........................................68

Figure 5-3: Names of parts referred to on a slit-lamp......................................69

Figure 5-4: Turnigy TGY-SM-8168R (left) and TGY-S810 (right)......................72

Figure 5-5: Joints and knobs activated on the slit-lamp...................................74

Figure 5-6: Clamp and aluminum bracket used to hold servos to slit-lamp.........75

Figure 5-7: Actuation of the rotational arms of the slit-lamp.............................77

Figure 5-8: Actuation of the light width adjustment knob................................78

Figure 5-9: Actuation of the chin rest height adjustment.................................79

Figure 5-10: Actuation of the slit-lamp height adjustment.................................80

Figure 5-11: Underside of the slit-lamp base with Omni-wheels mounted...........82

Figure 5-12: Actuation of the slit-lamp base with Omni-wheels..........................83

Figure 5-13: Full commercial laser and slit-lamp system (Zeiss).......................85

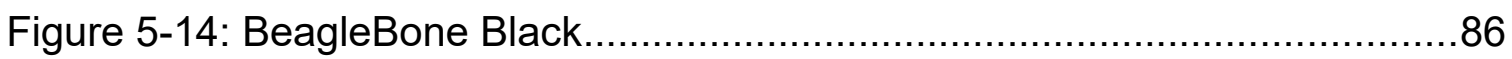

Figure 5-15: Adafruit 16 Channel 12-bit PWM/Servo Driver..............................87

Figure 5-17: Implemented control hardware for the first prototype....................89

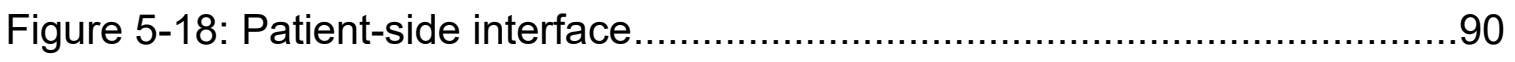

Figure 5-20: Portable physical control console ................................................96

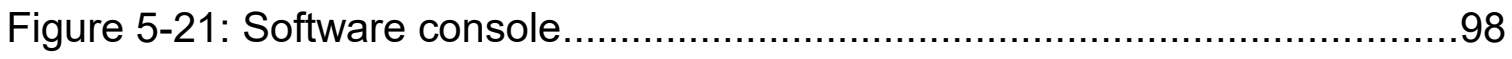

Figure 5-23: Compliant serial lens-holding arm. Image adapted from [121]......103

Figure 5-24: Parallel lens-holding designs. Image adapted from [24]...............104

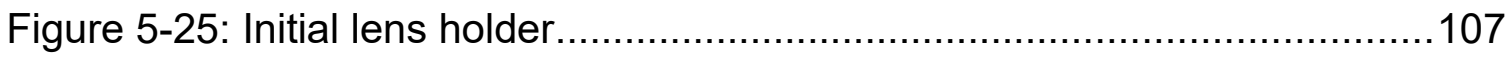

Figure 5-26: Initial lens holder actuation (from the patient's side of the slit-lamp). 
Figure 5-27: Pan-tilt mechanism for lens holder..........................................108

Figure 5-28: First robotic slit-lamp prototype - OphthBot 1............................109

Figure 5-29: Stepper-driven X-Y table for base movements...........................111

Figure 5-30: Geared servo interface to binocular and lamp arms....................112

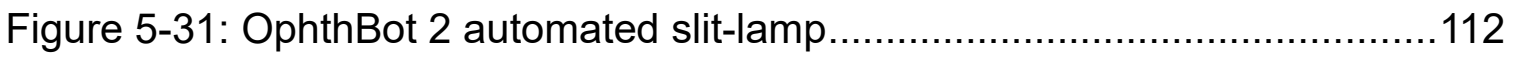

Figure 5-32: Design for OphthBot2 lens holder..........................................113

Figure 5-33: Close up of the implemented OphthBot 2 lens holder..................114

Figure 5-34: Full implemented OphthBot 2 lens holder..................................115

Figure 5-35: Complete OphthBot 2 system...............................................116

Figure 6-1: Robotic face without cover (left) and with cover (right).................119

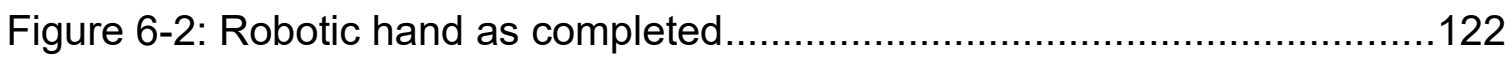

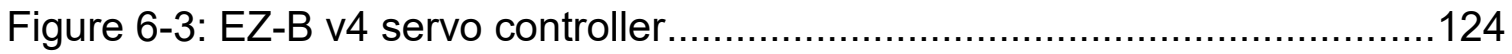

Figure 6-4: HCTeR Humanoid Rehabilitation Robot......................................128

Figure 7-1: Configuration of wheels and axles in base platform of

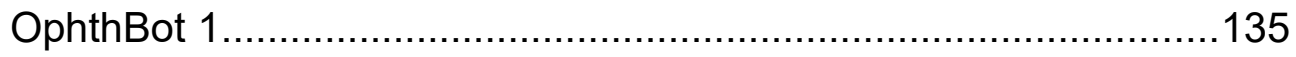

Figure 7-2: Dual-row Omni-wheel in profile .................................................138

Figure 8-1: Reti Eye practice eye by Gulden Ophthalmics.............................150

Figure 8-2: Graph of OphthBot 1 rotational component performance................154 


\section{ABBREVIATIONS AND ACRONYMS}

$\begin{array}{ll}\text { AROM } & \text { Active Range of Motion } \\ \text { ASL } & \text { American Sign Language } \\ \text { CP } & \text { Cerebral Palsy } \\ \text { DOF } & \text { Degrees of Freedom } \\ \text { GPIO } & \text { General Purpose Input and Output } \\ \text { mm } & \text { Millimeter } \\ \text { MPC } & \text { Model Predictive Control } \\ \text { PC } & \text { Personal Computer } \\ \text { pHRI } & \text { Physical Human-Robot Interaction } \\ \text { PROM } & \text { Passive Range of Motion } \\ \text { RCM } & \text { Remote Center of Motion } \\ \text { ROM } & \text { Range of Motion }\end{array}$




\section{INTRODUCTION}

Advances in the medical arts has increased rapidly over the past century. Though these improvements have been on many fronts, technology has been one of the leading factors in improving treatments. Robots are one of the newest types of technology to be applied to this field and their acceptability and use have increased dramatically in a short period of time.

Robots have numerous current and potential uses in health care. They can be used in hospital and clinical settings as well as in homes. Some uses include remote interactive patient consultations, home assistive care, cleaning, social companionship and activity, active prostheses, drug delivery, rehabilitation and surgery. These robots range from the nano-scale to large devices that occupy much of an operating room.

While all types of medical robotics are undergoing active research and advances, the work presented here focuses on robots used in ocular surgery and pediatric rehabilitation. While these areas seem different, there are many overlapping areas of research and concern. In addition to creating two prototypes for the ophthalmic robot and example robots for physical rehabilitation, a control system is developed that can be used to adequately control any type of robot with similar requirements. 


\subsection{Motivation}

Ocular disease and problems affect 285 million people worldwide [71]. It is estimated that up to $75 \%$ of worldwide blindness is preventable [106]. Two major reasons include high costs and the availability of ophthalmologists within easy travel distances.

Many of those lacking proper care are in rural areas or underdeveloped nations. While there have been attempts to provide care to these individuals such as utlizing the Flying Eye Hospital, the needs of many populations are not being met. Further, patients with conditions that require special expertise may not receive the best care when only physicians of other specialties are available to provide treatment.

Some solutions include increasing the localized access of care, increasing the number of physicians trained in specialized treatments, and reducing the operating costs of clinics and practices that perform ophthalmic services.

Robotic technology can solve many of these issues, though upfront costs can still be high. By utilizing standard communication networks, an ophthalmic robot can be controlled and monitored remotely by a physician in nearly any location. This allows care by specialized physicians to remote areas including rural villages, battlefields and places of scientific exploration. Patients can have more options in selecting who provides their care, such as a trusted physician in another location or an expert for a rare condition. Care can be 
provided by one physician or coordinated to simultaneously include any combination of specialists and medical personnel from a variety of locations. This not only improves patients' options and quality of care, but also provides a means of educating and training a pool of physicians about rare conditions and new treatment options.

Though the robots in each location will require an initial investment, some costs savings are possible in the long run. Physicians can use teleoperated robotic technology to reduce their travel time and costs. For instance, even if they are the only specialist in a region, they can work from home or a single nearby office by connecting to robots located at remote service points. These points could be shared medical offices, hospitals, clinics or even mobile units parked or scheduled in libraries, assisted living facilities, homes or any other appropriate location. They can also increase the number of patients seen daily whether local or spread over a wide geographical area by allowing the physician to nearly instantaneously switch from one robot to another. Physicians can operate in clinics located in areas where they may not have the desire to live or that may not fully support the income they wish to receive. By offering their services in a more affluent area at higher rates and also providing remote services in other areas at much lower rates, physicians can serve a wider range of populations while maintaining their desired lifestyle.

In addition, robots offer several additional advantages in many procedures including improved accuracy, endurance, hygiene, and dexterity. Hand tremors 
are a major issue in eye surgery since these movements in healthy individuals can be $312 \mu \mathrm{m}$ wide when holding an instrument steady and larger during motions [97]. The sizes of the structures in the eye are such that this is a significant proportion in some procedures. Robots reduce and eliminate these tremors so that components such as the lens holder and laser are held steady and stable [113]. A robot may also be able to hold a position and automate simple routine tasks, reducing the effort and strain on the ophthalmologist. In many cases, a robot can offer more degrees of freedom (DOF) and more ideal kinematics for performing procedures - especially because the structures of the eye are so small and scale of motion is very different than normal human movements. Finally, a robot can be covered and sterilized more effectively than a physician so that the occurrence of disease transmission is reduced.

In addition to costs, the other major inhibitor to implementing robots for this purpose is the safety of their use in direct contact with humans. This is especially a concern when a robot is being operated by a physician that is not on-site. Issues such as time delays and operation faults can lead to undesired consequences. A controller must be designed to not only be robust and faulttolerant, but it should be effective for a range of ophthalmic robots in order to increase adoption and reduce costs.

These issues and concerns apply to other areas where medical robotics are used remotely or with minimal supervision when interacting with patients. Use of robotics in physical rehabilitation is a second example provided here. 


\subsection{Goals}

This project had two distinct goals, though both lead to the ultimate outcome of having a fully-functional ophthalmic robot that can be safely used via teleoperation. In addition to the first goal of designing and building a physical prototype, the second goal was to develop a control system that is adaptable and robust for a variety of slit-lamp configurations. The control system was to be developed to be applied to other areas of medical robotics with pediatric rehabilitation robots being of the greatest interest. Specifically, this means that the control system can be used safely with little or no adaptation in a wide variety of human-robot interaction scenarios.

\subsubsection{Ophthalmic Prototype}

Goals for the primary prototype, dubbed OphthBot, include it being based upon a standard slit-lamp that offers the full examination and treatment form and functions with which nearly all ophthalmologists are familiar. This not only reduces the learning curve, but also can decrease the costs because the base structure is an off-the-shelf piece of equipment that has been around for over a century. Ideally, the robotic portion of this project was to be made into a retrofit kit to work on nearly any slit-lamp so that clinics can save money using what they already have and physicians can use the particular model in which they are most comfortable. The prototype was to demonstrate that a robotic slit- 
lamp can provide examinations and treatments from a remote location with only the aid of a nurse or medical assistant aiding the patient on-site.

\subsubsection{Control Theory}

Goals for the control system were to be able to operate an ophthalmic robot from a remote location using standard communication methods, safely operate despite time delays that may not be accurately known and may change quickly over time, automatically adapt and work accurately with various slit-lamps, and work safely in direct contact with patients.

Secondly, the control system was to be flexible and easily adapted for use in other areas of human-robot interaction. Specifically, a goal was to apply it toward robotic technology for pediatric physical therapy. The use in humanoid robots for modeling is the specific example provided as it limits the scope and has related uses to ophthalmic research.

\subsection{Significance}

The prototype ocular robot was the first to be earnestly developed for the market that is based on a tool very familiar to all ophthalmologist. It was the only robotic tool nearing commercialization at time of publication that offers both full-featured examination abilities as well as the ability to provide laser surgeries for some ocular conditions via a remote platform. Unlike specially- 
designed robots, this device was based on slit-lamps used by nearly all ophthalmologists for decades. Modified and simplified design allows for a system that can be purchased new and used in nearly any country and at various prices. This not only decreases the learning curve for the device and reduces the financial investment, but also allows the system to be easily customized to suit the needs and preferences of physicians.

The prototype rehabilitation robots bring novel improvements to technology as well. They provide a non-living, but animated and interactive platform to allow for training and testing of medical devices. They also can be controlled remote control to facilitate a more tactile and visual proxy experience to allow therapists or physicians to quickly interact with patients in distant locations. Next, humanoid robots can be used as a surrogate for a therapist to interact with children on the autism spectrum to allow for more interaction during most therapeutic encounters. Finally, low-cost humanoid robots allow for kinematic and dynamic adjustments so that the system matches the physiology and anatomy of the child it will help. This allows for very realistic and accurate modeling of the tasks as the client is able to perform them as well as showing validation and acceptance of the child's unique appearance and abilities. 


\section{ROBOTIC OCULAR SURGERY LITERATURE REVIEW}

Before getting into the specifics in ocular robot technologies, it is helpful to overview some of the key developments of robotic surgery in general. Afterward, robotic ocular surgery components are presented. Relevant issues and current state-of-the-art are briefly surveyed. After discussing laser use in ophthalmology, a brief discussion of current open issues supported by the preceding sections is provided.

\subsection{General Robotic Surgery}

Robotic surgery has been a reality since a PUMA was used in 1985 for a brain biopsy [61]. Benefits of using robots to perform surgeries include smaller incisions, better accuracy, tremor reduction [98], adjustments to scale [17], and higher mobility [54]. All of these things benefit the patient by providing a more directed surgery and faster recovery time. Robots can also reduce the personnel involved in a procedure and reduce the operation time, cutting costs for both patients and medical providers [74].

It should be noted that it is not typically the intention of robotic surgery to completely replace highly trained medical personnel including operating physicians. Instead, robots are a powerful tool intended to improve a surgeon's abilities utilizing the benefits listed above. Given the increase in abilities, it is 
inevitable that some personnel will no longer be needed such as multiple assistants to hand over instruments or move a camera. Instead, the surgeon has control of these instruments via the control console [62]. The use of robots may reduce the number of errors in the operating room [17] and can decrease some costs by eliminating some assistants [74]. However, robots are not able to make medical judgments, can malfunction and have their own maintenance costs [87], and so they cannot in the near future replace skilled physicians, nurses, nor all assistants.

A future benefit is the ability to perform surgeries remotely via teleoperation [94]. While this is already the case in some instances either directly [69] or by teleproctoring [29], it is not in routine nor widespread practice. This feature allows a specialist in one location to control a robot and perform surgery on a patient in another location whether it is across the room or across the globe [43]. This may be necessary if the patient is in critical need and does not have time to travel or wait for a specialist to arrive. Teleoperation may also be used to remotely provide first-response care to soldiers and civilians in areas not easily accessible due to geography, politics, disasters, and war. It could also be a tool to provide care when distances are too great to travel for any specialized care, such as during a space exploration mission.

Robotic surgery allows for procedures to be performed by one or more physicians located right beside the patient or from a distance. This not only allows for more opinions and options for the patient to consider, but can also 
provide a means for training surgeons about rare conditions and new procedures. Information can be recorded to be used in virtual reality training exercises and eventually to create a virtual training library.

While some of these benefits are not fully realized as of now due to costs and communication abilities, robots were used in over 20,000 surgeries every year as reported for 2010 [48] and over 360,000 procedures in 2011 [41] with growth continuing. There are hundreds of robotic surgery systems in various stages of development [48] [87]. By far, the most popular surgical robot currently on the market is the da Vinci by Intuitive Surgical. The da Vinci is commonly used for certain types of surgery including hysterectomies, prostate care, and thoracic surgeries [119]. Other popular surgical robots fully in use target orthopedic surgeries. These include MAKO Robotics's RIO for knee and hip replacement surgery [40] and Mazor Surgical's SpineAssist and Renaissance [103] for aiding spinal surgeries.

Other surgical robots are nearing mainstream use. One of these is the Raven surgical system to compete with da Vinci with a much smaller size, weight and price tag [65]. It can also accommodate additional features such as the ability to pump blood and maintain circulation during surgeries. There are numerous other systems as well that are targeting brain surgery [72].

While current robots are primarily used for intracorporeal and orthopedic surgeries, there are other areas where robot technology can be employed. One such area is in the eyes. 


\subsection{Robotic Ocular Surgery}

In this section, some fundamentals of ophthalmology are provided to aid in the understanding of the robotic device and procedures it performs. The current state-of-the-art in ocular robotic surgery is also detailed.

\subsubsection{Ophthalmology Basics}

Before delving fully into details of robotic eye surgery, a brief introduction to ophthalmology is helpful. Basic terms and structures in eye care, as well as some tools used in this area of medical practice are described in the subsections that follow.

\subsubsection{Eye Anatomy and Physiology}

The eye is a round organ approximately $24.2 \mathrm{~mm}$ in diameter in an adult [4]. It is composed of several layers and includes numerous parts that aid in the retrieval of visual information from the environment. A diagram of major components of the eye are shown in Figure 2-1. 


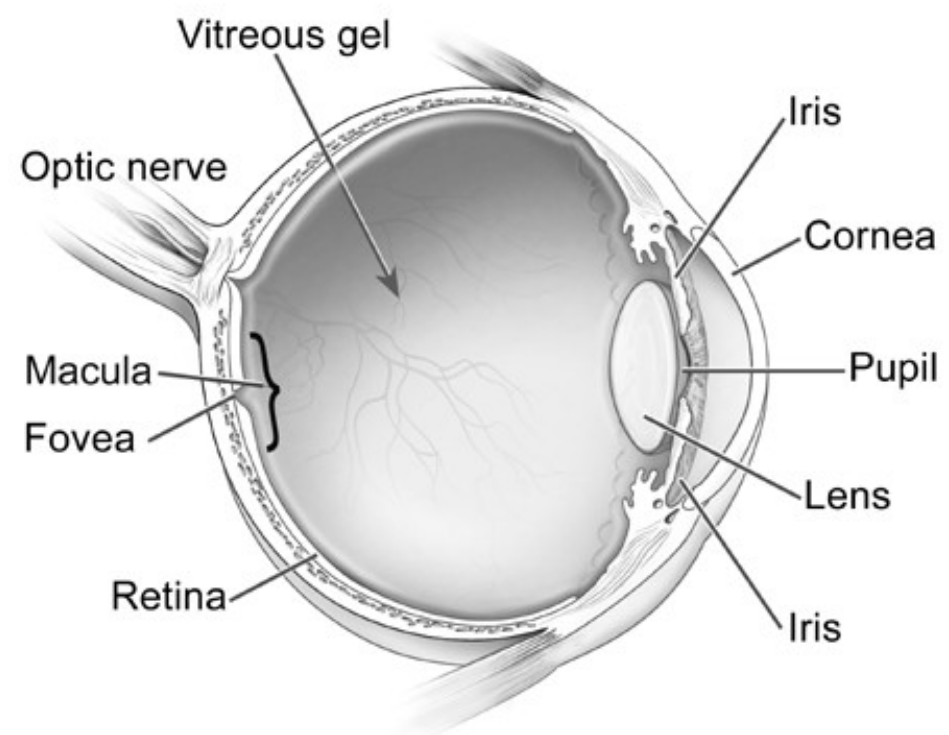

Figure 2-1: Eye Anatomy [from nei.nih.gov]

\subsubsection{Examination Procedures and Tools}

A sample of basic equipment used in eye examinations include tonometers, phoropters, retinoscope, refraction boxe and the slit-lamp biomicroscope.

The main instrument of concern for this work is the slit-lamp. This device was first developed in 1911 [115]. It is capable of a wide variety of examination tasks and can also be fitted with accessories to accomplish a greater range of uses. The slit-lamp is mainly useful for examining the anterior of the eye and can also examine the posterior segments with appropriate lenses [115]. In addition to examinations, the slit-lamp also lends itself to be used in treatment procedures as well. This is because a laser can easily be integrated via an adapter to be used in various ways as mentioned in the following section. 
A slit-lamp can come in a variety of configurations. Most commonly, they are split into two categories: a Zeiss-type or a Haag-Streit-type. There are also portable models that are handheld, but due to their limited options and lack of a stationary base, those are not of primary focus here.

Slit-lamps have two major working components, the slit illuminator and the microscope. The light is transferred through optics via the Kohler illumination principle [115] and is directed at the eyes. Direct illumination is avoided in modern instruments so that the image of the source is not reflected in the eye. Through the use of electronics, optics, and mechanical adjustment, several aspects of the light output can be adjusted and comes out as the desired slit of light. These adjustments include the light intensity, height, width, color, and angle. The microscope is attached to a swinging arm and can manually be adjusted for angle. Magnification and focus are also adjustable. The swinging movements of the lamp and microscope arms are almost always coupled so the entire device is parfocal and focus at the same point. In Zeiss-type slitlamps, the light comes up from a source below the output point and usually a Galilean magnification changer is used in the microscope. In Haag-Streit-type slit-lamps, the light source originates above the output point and a Grenoughtype of microscope may be used.

The slit-lamp also includes other parts. Both the lamp and microscope portions are connected to a base platform. This base platform sits on the table surface connected by a cross-slide and is able to move back and forth as well 
as left and right. Located so that an eye can be placed at the focal point, a head rest assembly including a height adjustable chin bar and forehead rest, is attached to the slit-lamp base. This is where the patient's head is placed during the exam. A small manually-moved focus light may be attached to this assembly to provide a means for the ophthalmologist to direct the patient's gaze direction.

\subsubsection{Treatment Procedures and Non-Robotic Tools}

Laser eye surgery is typically performed by the ophthalmologist manipulating instruments and a laser by hand while looking into a binocular

microscope. The procedure success depends upon the dexterity of the physician during what can be long and arduous procedures [102]. Due to the risk of unintended damage from a hand tremor, patient movement, or the fatigue and strain on the surgeon's eyes and hands, robots are a good solution to improve existing instruments [113]. Coupled with robotic technology, the ability to track along any eye movement is needed. In many procedures, it is preferred that the eye is not anesthetized and can move normally.

Lasers are used extensively in eye surgery for a variety of procedures. They have many benefits in ocular use including not requiring physical contact, being precise, and allowing for many parameters to be customized. Laser parameters that can be controlled include the wavelength, intensity, pulse duration, and spot size [68]. Different lasers can be used for different purposes 
in order to utilize different ranges of wavelengths and energy [34]. The wavelength is important to ensure effective absorption by the tissue being treated and the energy is important in ensuring the correct amount of tissue is affected.

Most well-known are the refractive surgery uses, such as with vision correction in LASIK procedures. In these instances, excimer lasers ablate small portions of the cornea so that the shape is altered to correct visual distortions and improve vision [114]. Lasers can also be used to treat glaucoma by lowering the intraocular pressure and to treat cataracts [68].

Lasers can also be used on the posterior segments of the eye, particularly in retinal procedures. They can help treat conditions such as diabetic retinopathy, vessel occlusions and retinal breaks [68]. These conditions are treated by focal coagulation of the retinal pigment epithelium (RPE). Points can be targeted one at a time or, on some laser systems, certain patterns can be selected for faster treatment [6]. One major issue with retinal procedures is tracking movements in the back of the eye so the laser treats the desired points. Failure to do this can cause unintended damage including blindness if the wrong target is hit.

There have been some investigations on tracking the retina using various image processing tools [55] that have proven to be successful in clinical trials [50]. These laser systems are themselves robotic in nature since they are precisely controlled to perform at given locations at a variety of parameters that 
can be adjusted as previously mentioned [114]. Still, fully robotic systems have been investigated for ocular use for years now and those are detailed in the next section.

\subsubsection{Current Ocular Robotic Systems}

Ophthalmic uses of robotics are currently under development including the Eye-RHAS (Eye Robot for Haptically Assisted Surgery) and PRECEYES [42], IRISS (Interocular Robotic Inverventional and Surgical System) [92], and Steady Hand Robots [78] [113]. By most accounts, certain procedures such as LASIK are also considered robotic because mirrors or fiber optics are actuated to quickly direct the laser beam at the desired target on the eye, even while the eye is moving. The surgeon does little intervention once the procedure is started [87].

Robots have been tested for ophthalmic uses since at least 1992 [37]. An early 6-DOF parallel robotic arm [36], simply referred to as "The Eye Robot," is shown in Figure 2-2. It was not only used in live animal experiments, but was also was used for testing the feasibility of adding haptic feedback [37]. Another early system is shown in Figure 2-3. It is unnamed and was created to experimentally prove that robotic technology opened up the possibility of a wider range of procedures that could be performed within the eye, particularly vitreoretinal drug delivery [122]. 


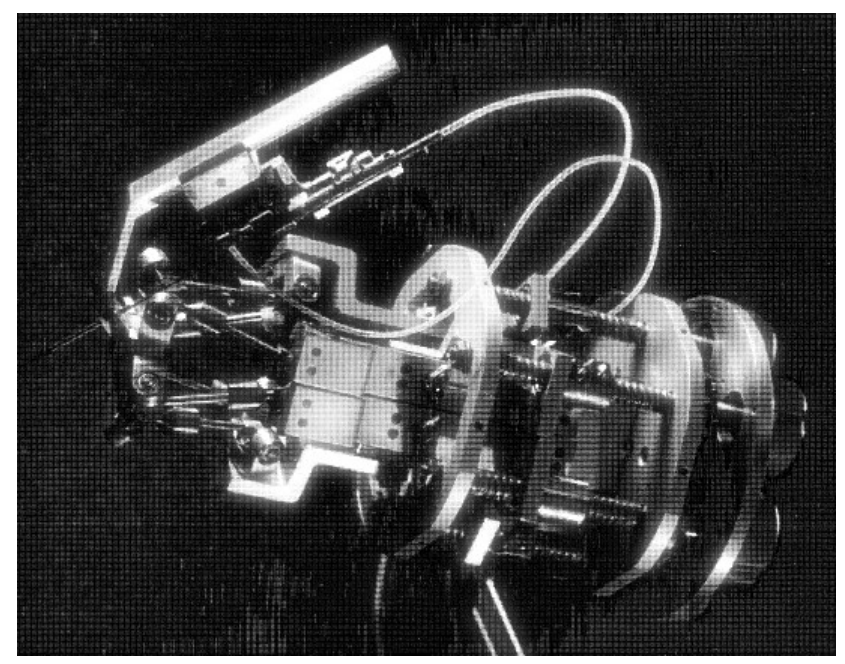

Figure 2-2: "The Eye Robot" [37]

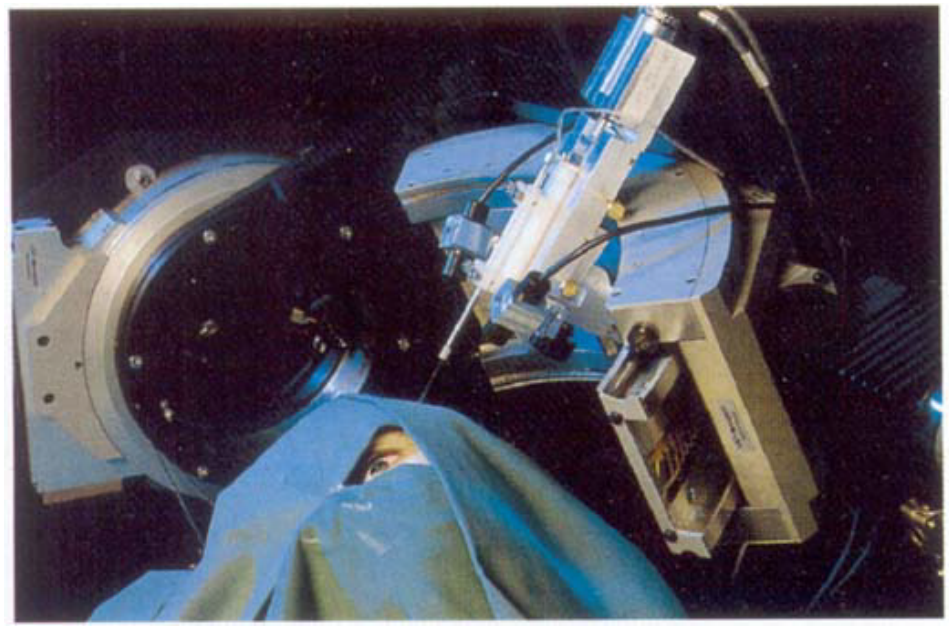

Figure 2-3: Experimental ocular ultramicrosurgery robot [122]

The Steady Hand Robots have been created at Johns Hopkins University for vitreoretinal surgery. They aid in the control of an inserted instrument as shown in Figure 2-4. Force sensors are used to detect hand motion in order to move the robot arm with the surgeon, while eliminating tremors and stabilizing 
the motion. The first prototype, Eye Robot 1, had a positioning precision of five microns, but many other limitations. The researchers worked to obtain the specific goal of performing retinal vein cannulation, which would allow for direct targeting of drug delivery to this structure [78]. Eye Robot 2 [113] was built to improve upon some of the limitations of its predecessor. Rejected design decisions such as a six-bar mechanism in the manipulator were reconsidered and implemented to give a greater range of motion rather than a more compact design.

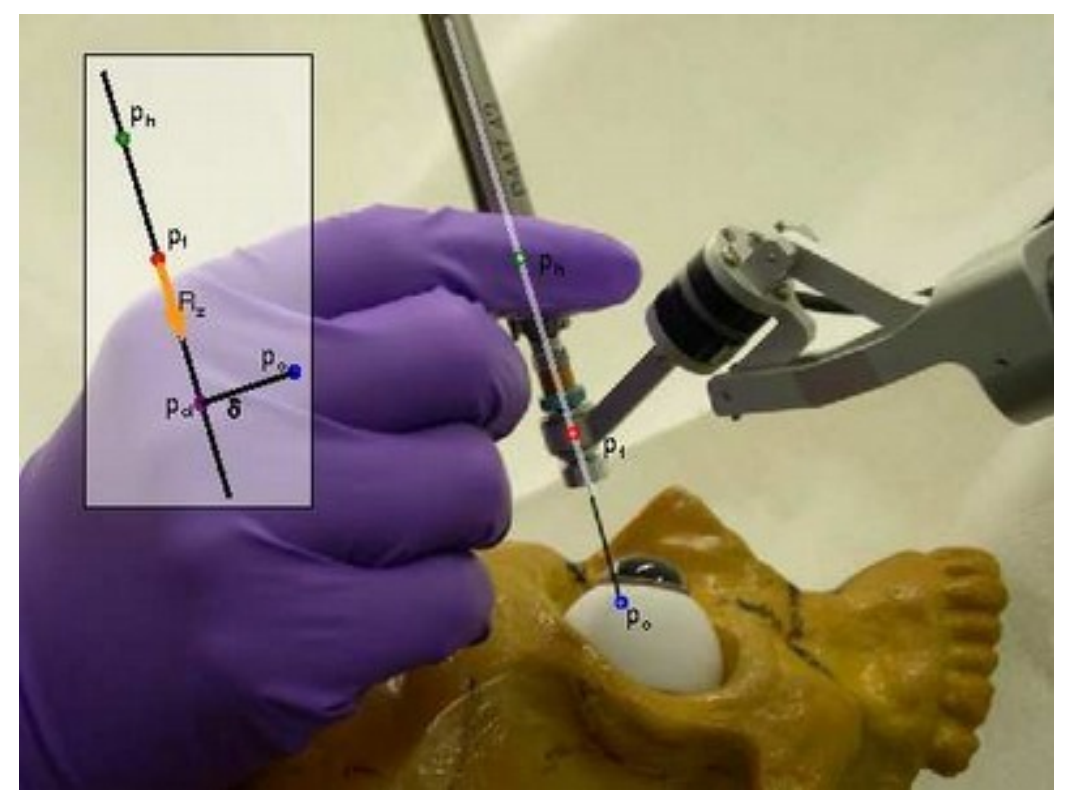

Figure 2-4: Steady Hand Eye Robot 1 [78]

Other designs have been introduced that include various parallel robotic arms. These are popular due to the high stiffness and accuracy. One of these 
devices uses two 6-DOF parallel robotic mechanisms to control the position of a halo as shown in Figure 2-5 to gain very high precision [117] [32]. Another device (Figure 2-6) uses a master/slave system and has been specifically designed for vitreoretinal surgeries and has proven to significantly reduce the effect of tremors. It has been carefully designed for the demanding specifications and sterilization abilities to ensure commercial viability [83].

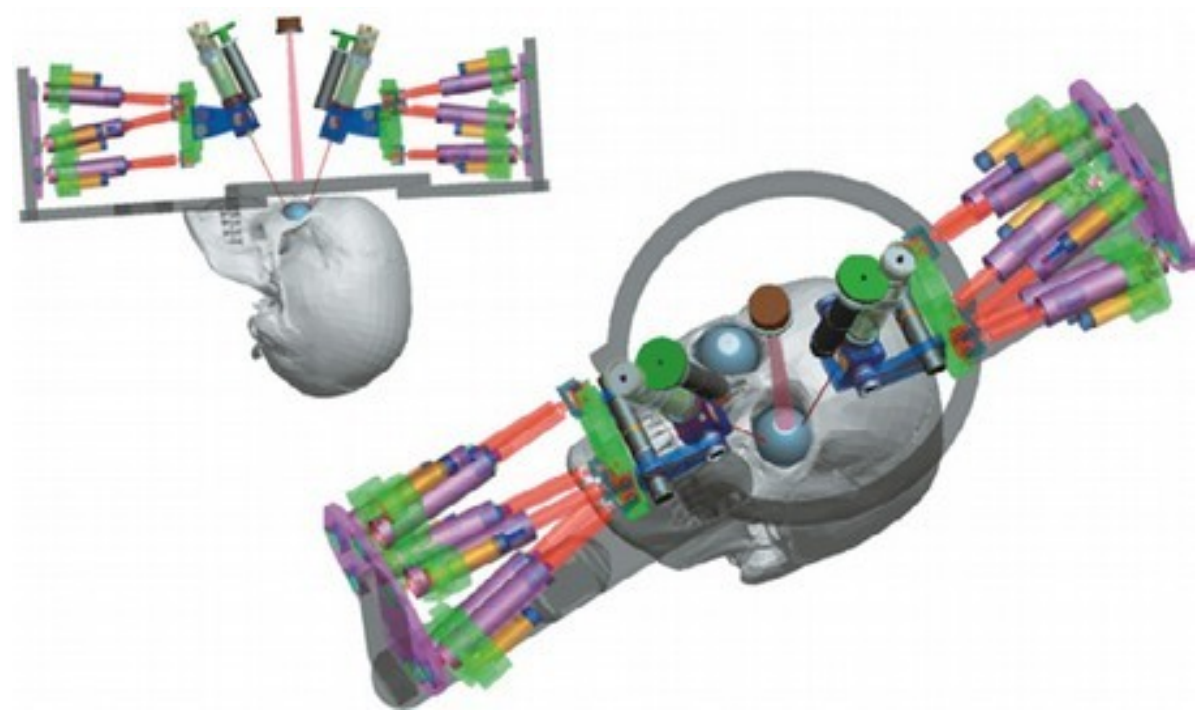

Figure 2-5: Design for a surgical system using a halo positioned by two robotic arms [32] 


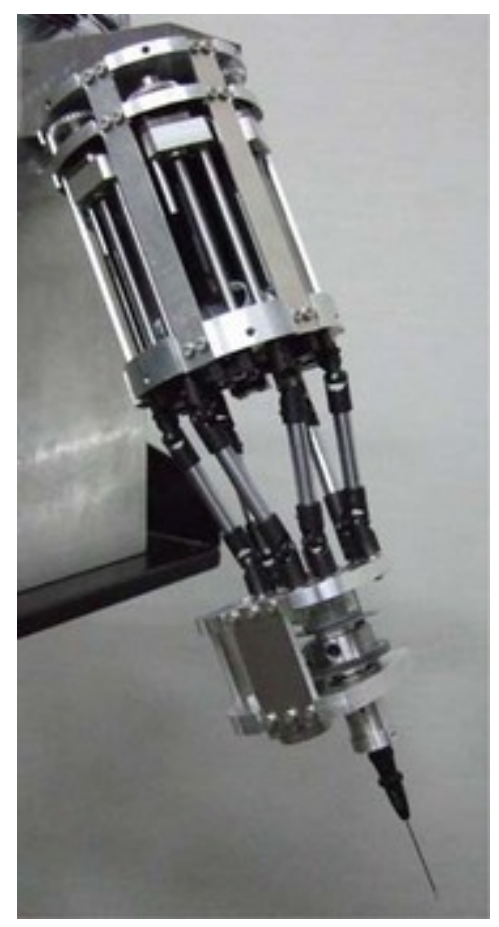

Figure 2-6: A parallel robotic arm for vitreoretinal surgery [83]

A novel design uses tiny MEMS-controlled pneumatic actuators to mimic the motion of a human hand. Tiny bladders are inflated and deflated in order to make the 4-mm long fingers curl or straighten as shown in Figure 2-7. The system is able to lift and manipulate retina tissue and so shows promise for performing as micro forceps in ocular procedures [48]. Another novel concept is the OctoMag. It utilizes eight electromagnets set at predefined angles to direct an untethered metallic micro-robot similar to that shown in Figure 2-8 to perform desired tasks [70]. 

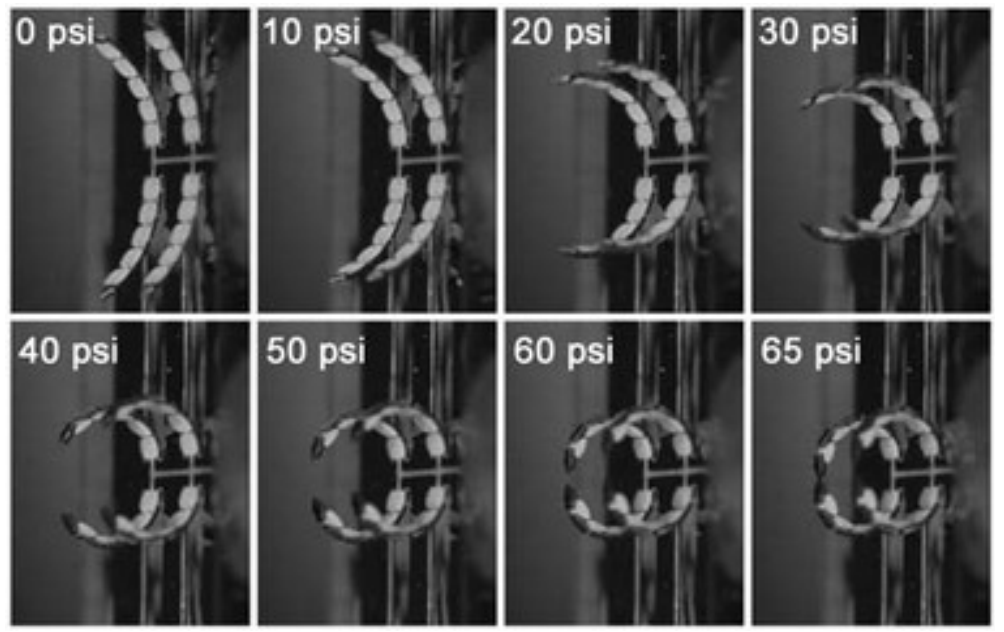

Figure 2-7: Microhand forceps closing under increasing pressure [48]
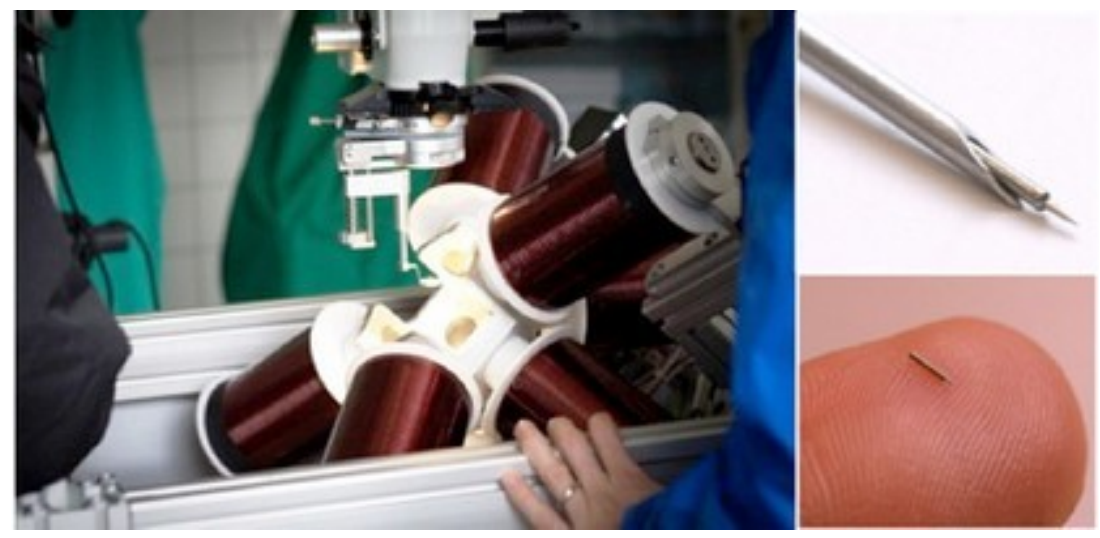

Figure 2-8: OctoMag micro robot [70]

IRISS was designed with the intention to perform all types of intraocular surgeries [92]. The robot serves as a slave and is controlled by a similar master console. The design focuses on maintaining the remote center of motion $(\mathrm{RCM})$ via both hardware and software controls. Each stage of the robot maintains a RCM as can be seen in Figure 2-9. 


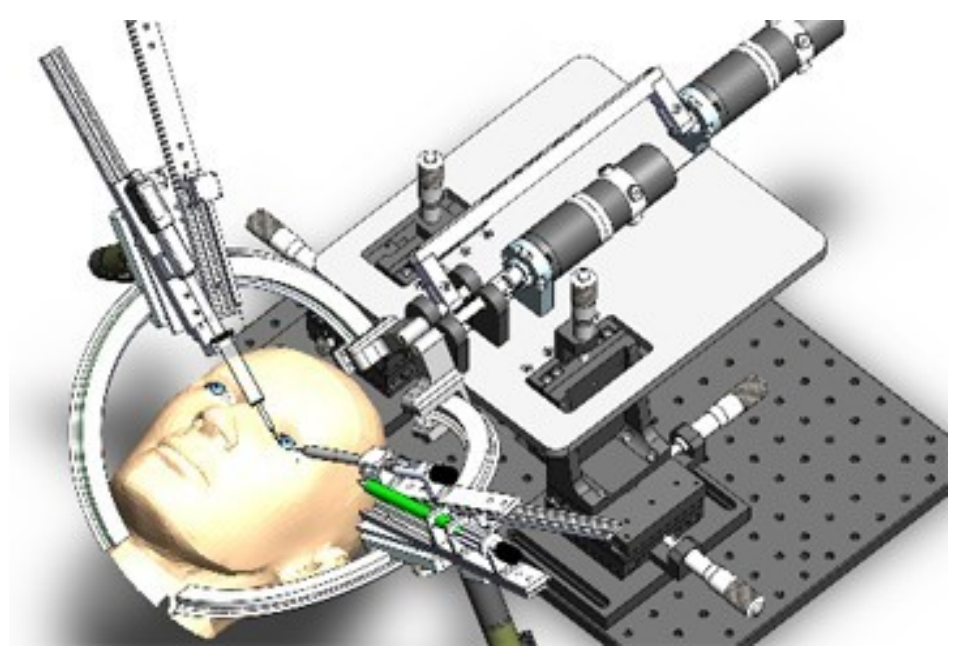

Figure 2-9: IRISS eye surgery robot [92]

PRECEYES is a company that started as a spin-off from university research in the Netherlands that included work on the Eye-RHAS control console [42]. The company hopes to be the first in the world to reach the market. Toward this goal, they are already the first robotic system used to actually perform eye surgery [116]. The system, shown in Figure 2-10, consists of two arms that help guide the surgical instruments. The arms reduce tremors and also provide haptic feedback. 


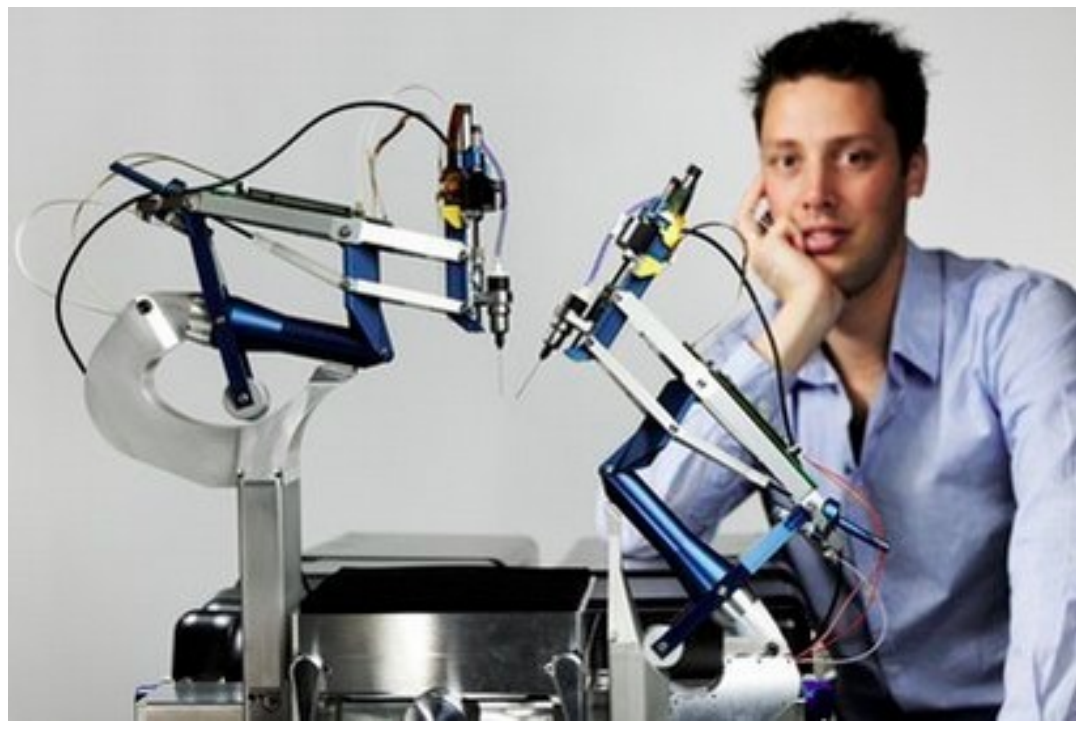

Figure 2-10: PRECEYES prototype [42]

In addition, proposals to use da Vinci system (Figure 2-11) have been presented. As it was with general robotic surgery, the high cost of the da Vinci system is a problem in quickly gaining widespread use of this system for ophthalmic uses. However, discounting this with the assumption that many medical centers now have these robots available anyway, it is interesting for many to try to use these already-mainstream robots for ever more specialized tasks. An early test showed that it was feasible to use the da Vinci for eye surgery, but it was much slower than using traditional instruments [111]. It was suggested that developing smaller manipulators for the da Vinci would be beneficial. This sentiment was echoed two years later in [9] when the da Vinci system was used to test whether a more intricate procedure (a penetrating keratoplasty operation) could be performed within a human head. While the 
procedure was successful, it was determined that the da Vinci was not optimized for performing eye surgeries. Vitreoretina operations are never attempted with the standard da Vinci since the 10-mm diameter manipulator arms are much too large to safely enter the sclera [112]. In order to address these issues, a research team has developed a parallel robotic manipulator called the Hexapod Surgical System (HSS) to be placed onto the da Vinci as shown in Figure 2-12. This system combines two proven technologies so that a complete eye surgery can theoretically be performed with both intraocular and extraocular tasks [10]. Further work is needed to fully realize the use of a da Vinci robot for ocular surgeries, however.

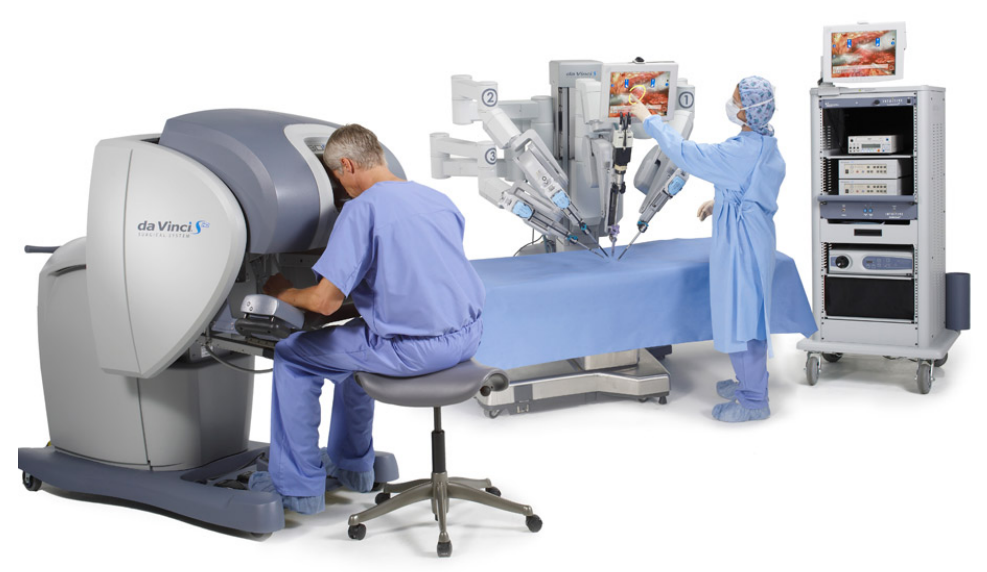

Figure 2-11: da Vinci surgical robotic system [99] 

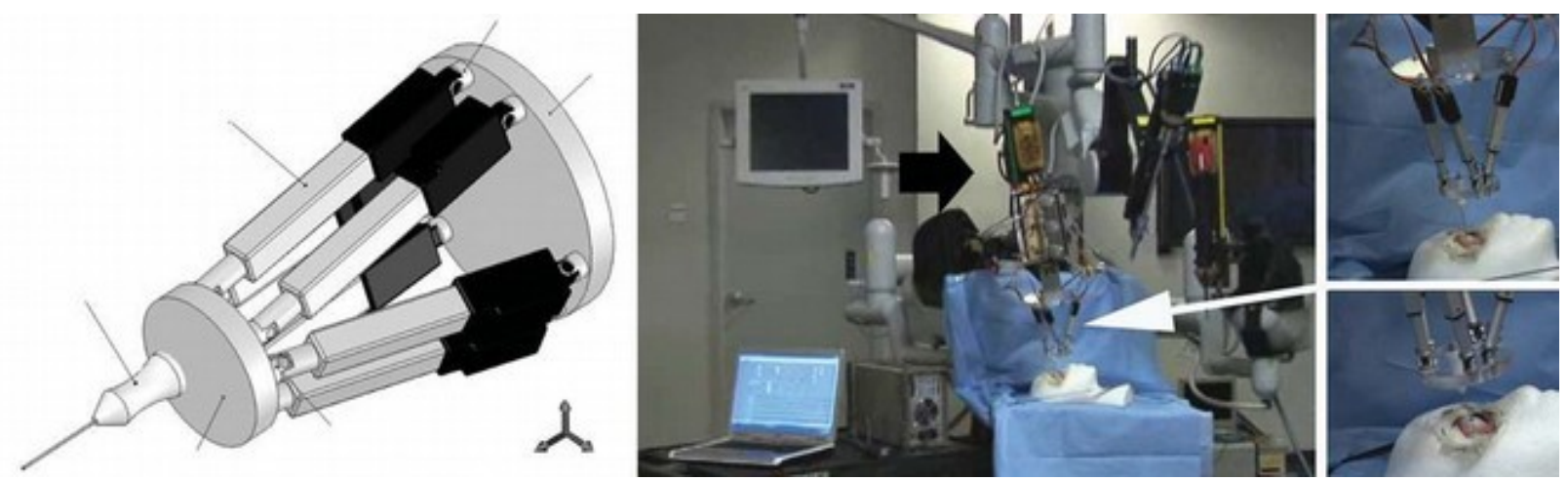

Figure 2-12: Hexapod Surgical System for micro-macro manipulations [10]

\subsubsection{Current Ocular System Technologies}

In this section, some current technologies, components and issues relevant to ocular robotic surgery and examination systems are reviewed.

Ocular robotic surgery has a few key aspects that make it different from other types of robotic surgery. One of these is the issue of scale. Small errors in position that are acceptable in general internal surgeries may not tolerable in eye surgery. The components of the eye are very small and delicate compared to most other organs such as the heart, bones or reproductive organs. In addition, some structures such as the retina are unable to fully heal if damaged and are also hard to access both visually and with instruments [78]. The second key aspect is that any instruments that enter the internal parts of the eye must not produce any forces on the outer eye surfaces. Small tensions that may be acceptable on skin can severely and permanently damage the sclera other vital components. Not only can this cause unnecessary scarring, but it can lead to vision loss or blindness. 
Avoiding forces at the point of entry is a problem that is being tackled by a variety of means. One is by manipulator design. This involves implementing a remote center of motion ( $R C M)$ where the instrument has a motionless point where the instrument sits at the sclera or other point of entry. The manipulator moves below this pivot point as the control mechanisms are worked above. An early design included 4-DOF manipulator, though it slowed the physician's ability to perform tasks [38]. Another team utilized the concept of this system with a robot manipulator and was able to show that precision and dexterity could be greatly increased [78]. They also studied the phases of retinal surgery and determined the minimal number of DOF needed for each step.

Tissue damage due to excessive forces from instruments is a great concern in many types of surgery, particularly ocular surgery. In the case of eye surgery, most of the force thresholds are too small for surgeons to manually detect as they are outside of the human sense of touch [39]. Currently, surgeons use visual inspection to detect and avoid excessive force. However, by implementing force sensors, these micro-forces can be sensed and the information can be used to aid physicians during delicate procedures. This is also a crucial step in order to make certain maneuvers autonomous in the future.

One group of researchers have worked to measure tool-tissue forces distally [109]. By using force sensors in the shaft and transmitting information via fibers optics to the tool handle outside of the eye (see Figure 2-13), 
accurate vitreoretinal force information is obtained with a minimal increase in the inserted portion of the instrument. They have noted that placing force sensors in other positions has severely decreased the accuracy and amplitude of the readings.

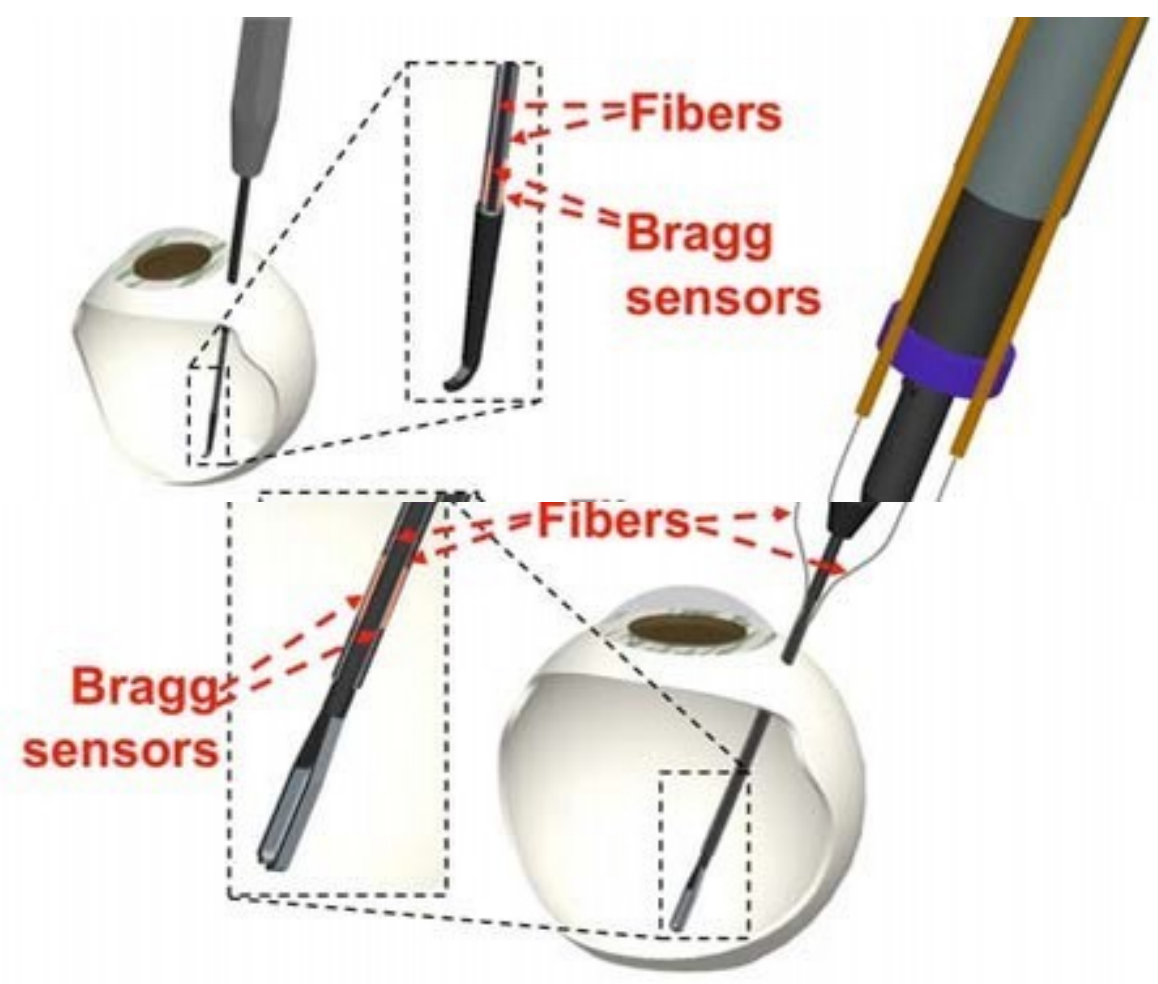

Figure 2-13: Fiber optic force sensors [109]

One issue with control of robotic manipulators in eye surgery is the guidance of surgical instruments. The Steady Hand Robots hold instruments and move according to force applied by the surgeon's hand via admittance control, reducing tremors in the process [78]. It also offers the great advantage 
of holding the instrument position should the surgeon let go, allowing for the surgeon to rest and avoid fatigue during certain procedures such as the injection of medication into a vein.

As mentioned before, one of the problems with inner eye surgery is the need to avoid stresses on the tissue at the insertion point. Software, alone or in addition to hardware, can be used to solve this issue. Eye Robot 1 used software to compensate for simplification of a six-bar mechanism to a compact slider-crank mechanism in order to keep the RCM stationary. In contrast, Eye Robot 2 uses a more complex mechanism to maintain the RCM and uses control software only to improve the stability of the RCM. Information from force sensors aid the surgeon by encouraging movements that cause the least amount of force on the tissue. This algorithm is called "micro-force guided cooperative control" and it showed promise as a solution when tested on raw chicken eggs [113].

This chapter completes an overview of current surgical ophthalmic robots. Some background on an existing robotic device that can be used for ophthalmic exams and additional control issues will be discussed in Chapter 4 . 


\section{PEDIATRIC ROBOTIC THERAPY LITERATURE REVIEW}

This chapter discusses the state-of-the-art of robotic technology for pediatric physical therapy. It provides the foundation of another application for the control systems developed later.

\subsection{Robotics in Physical Therapy}

Robots can be used in physical therapy in two ways. One form is to use the robot for assisting daily living tasks and the other is to use in physiotherapy. Assistive examples include advanced prostheses [22], mobility devices, and aids for daily tasks [67]. Devices used for physiotherapy include aids for the neurophysical rehabilitation of an individual due to a disability or accident that may be used for periods of time, but not usually permanently. In some cases, a robotic device may perform both tasks if programmed to switch functions. This work will focus mostly on robotics in physiotherapy.

\subsubsection{Physical Therapy Basics}

Over the past two decades or so, the use of robotics in neurorehabilitation has become a reality. In this application, robots assist in the physiotherapy of individuals who suffer from disabilities due to stroke, Parkinson's Disease, Multiple Sclerosis, spinal cord injuries or other motor impairment conditions. Where traditional therapy may last only a short time due to the high costs and 
limited availability of physicians, patients can experience further improvements if therapy were to be continued [96]. With the amount of time permitted for rehabilitation by insurance companies in the United States decreasing, it is necessary to find ways to make rehabilitation time more effective [13] or allow patients to continue less expensively without insurance.

The use of robotics makes long-term rehabilitation a possibility. They do this by allowing patients to work without the constant presence of a therapist, and by reducing the costs by performing the routines at home. Though the upfront costs are something to consider, virtual visits with therapists can save money directly with lower session rates. The savings associated in the reduced number of trips to a therapy office, both in money and in time, could also help to offset the initial cost of a home device. In some cases, it has also been proven that long-term robotic therapy in a hospital setting doesn't cost more than traditional long-term rehabilitation care [47].

Another important factor in the design and marketability of robotic therapy devices is the user acceptance of such devices. Early in the research of physical therapy robotics, it was found that there was some apprehension toward using robots, both from patients and their therapists [26]. After using the robotic aides, however, the apprehension mostly dissipated. Other research has shown that the use of robotics is readily accepted by patients, but not necessarily by the therapists. While patients may work harder and enjoy simple exercises more, therapists may find that they themselves are bored or 
have less control. Acceptance is less of a focus in current research trends. With robots being used in homes and offices for vacuuming as well as the surge in robotic surgery, the general population has become much more accepting of the use of robotics. In fact, robots have a reputation for being fun and this could allow for more interesting therapeutic exercises to be performed. Integrated games and challenges in particular make the interface very familiar to younger patients.

The validity of the use of robotics in therapeutic improvements has been investigated. The use of repetitive motions to regain motor capabilities has long been used and has been shown to be helpful. Sometimes therapies begin with a passive-assistance technique where the therapist moves the patient who passively allows the motion. Many therapy techniques use an activeassistance technique where the patient tries to perform a task and is aided, when needed, to completion. This type of therapy is the most predominate in research, possibly because it is the most natural for robotic implementation [96]. Another technique used is the active-constrained mode of learning where a user's movement is halted if a deviation from a correct movement is made, but allowed otherwise.

Early therapy is nearly universally accepted as being better than delaying therapy [15]. In general, it is agreed upon that long-term rehabilitation continues to improve the condition of the patient. However, after the first several months, the results are not as evident and progress more slowly and so 
insurance companies and sometimes patients decide to discontinue further treatment. Robot-aided neurorehabilitation in particular has been shown to indeed affect motor learning [56] and motor capacity [59] through studies using the MIT-MANUS robot. There is debate, however, on whether robotic-aided therapy is significantly better than traditional therapy [96]. Though it is clear that robotic-aided therapy works, it may not work better than traditional therapy with a human therapist alone. Robotic devices must provide the correct type and amount of assistance to reach their full potential and the perfect treatment depends upon the individual patient as well as the severity and nature of the impairment. Robotic therapy could provide more motivation for the patient, however, if tailored to the interests and goals of that patient.

The effectiveness of robotic therapy at home is yet to be determined. One review study found that the outcome of non-robotic home therapy was similar to that found non-robotic in-office care [11]. Therefore, if a robotic device can achieve the same results as a therapist working alone, then it can be presumed from these studies that there would also be no noticeable reduction in progress by using a robotic device at home rather than in an office. In fact, it may soon to be seen if automated robotic devices can improve therapy at home as they can motivate patients as well as enforce and enhance proper form of therapy activities even when the therapist is not physically present.

A unique benefit to robotic physical therapy is the quantitative feedback that they can provide. Much of the time, traditional therapy tends to use somewhat 
objective measures in determining a patient's current level of progress [95]. Even in cases when quantitative measures are used (such as speed, distance, or strength of motion), these measurements may not be consistently measured by all therapists. Robot-aided rehabilitation systems could be outfitted with sensors and provide consistent and accurate information about patient performance regardless of the therapist.

\subsubsection{Current Physical Therapy Robotic Systems}

Robotics have been proposed and used in neurorehabilitation for over two decades. Just a few examples are mentioned here. MIT has been developing the MIT-MANUS [57], shown in Figure 3-1 below, since 1989. This system is used in upper-limb rehabilitation. It is the pioneering low-impedance robot device that allows it to be backdrivable. Impedance control $[44,45,46]$ allows the patient to move the end-effector of the robotic arm to perform a task using the video screen and only feel a reaction force when necessary, such as to provide haptic feedback. It has been used in studies and shown to provide better and more long-lasting results over non-robotic therapies [58]. It has been commercialized under the name InMotion [49]. 


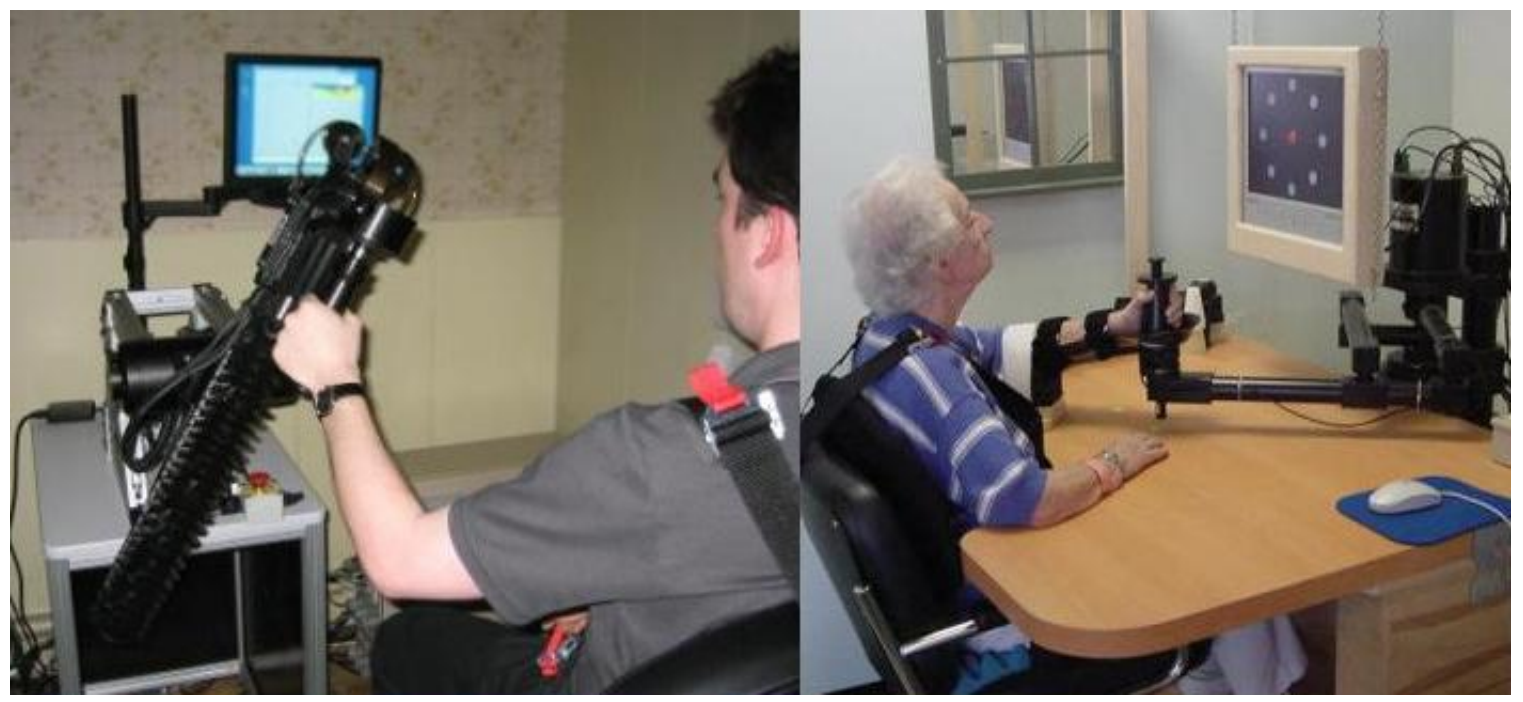

Figure 3-1: MIT-MANUS [57]

Another system that uses an industrial robot arm is the Mirror Image Movement Enhancer (MIME) device. This device is primarily focused on stroke victims or those with injury to one side of the body. In this system, the patient's good arm is used to move a passive robotic arm, which then acts as the master control of a slave robotic arm that moves the impaired arm [15]. The masterslave configuration gives the patient control over the movements and allows him or her to direct their efforts. It is also a safety feature in that the system will only move in a way that mirrors the ability of the non-impaired side of the body and so can not move the patient into unnatural positions. In addition, since the therapy is patient-guided, the patient can discontinue or avoid motions that are uncomfortable. The system is shown below. 


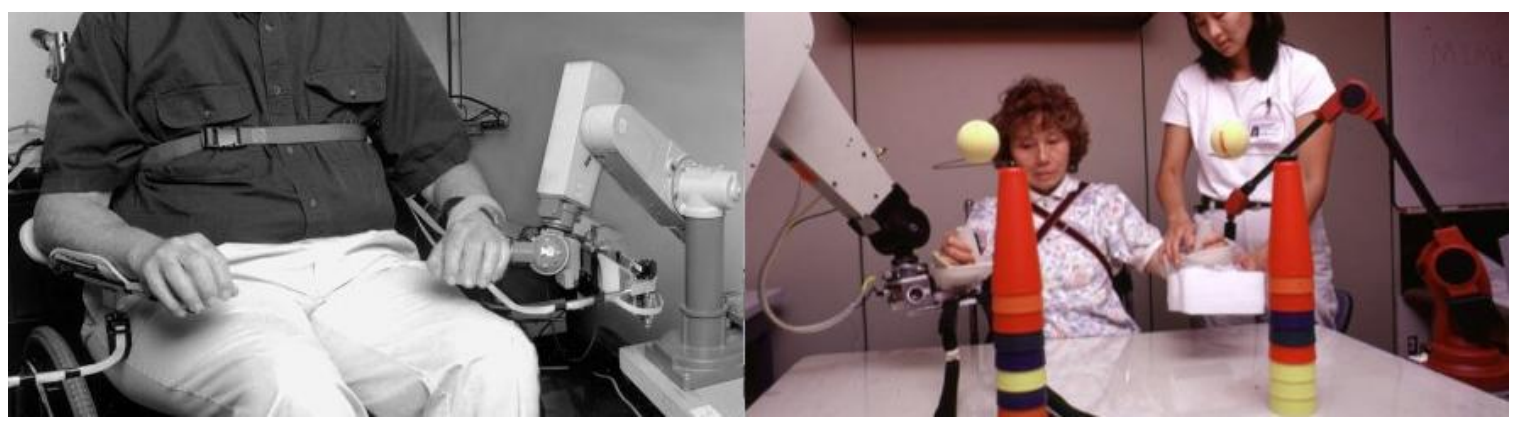

Figure 3-2: MIME Project [15]

Cable-driven devices are also proposed for use in upper-limb neurorehabilitation. Cables have inherent flexibility and are light so they don't risk inures from contact in the same manner as industrial robots. They can become entangled, however, or provide less accurate movements. A barrier between the patient and the cables is one method to ensure safety. This was done in a previous work by this author [81] and is shown below.

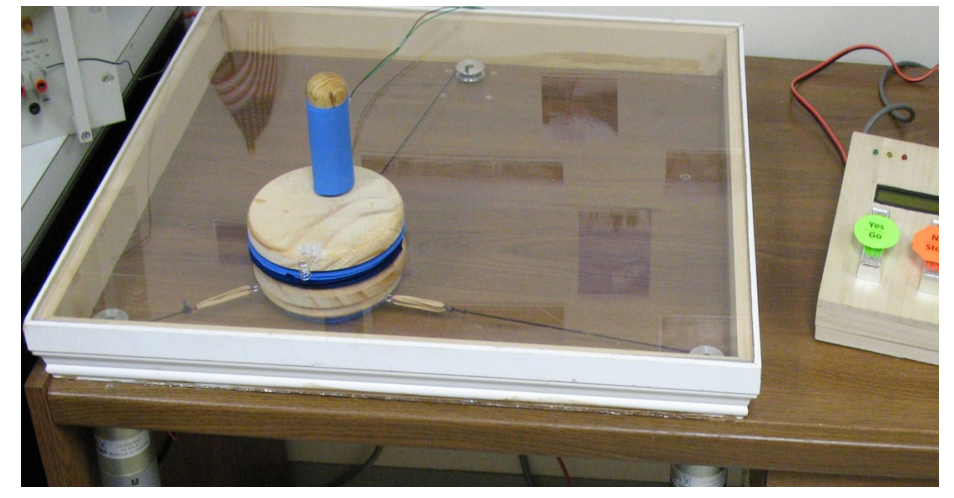

Figure 3-3: Cable-driven rehabilitation robot [81] 
ReWalk is a therapeutic system that is being used in centers to help patients regain lower-limb mobility [123]. It is the first FDA approved exoskeleton and is currently on the market and in-use at some clinics.

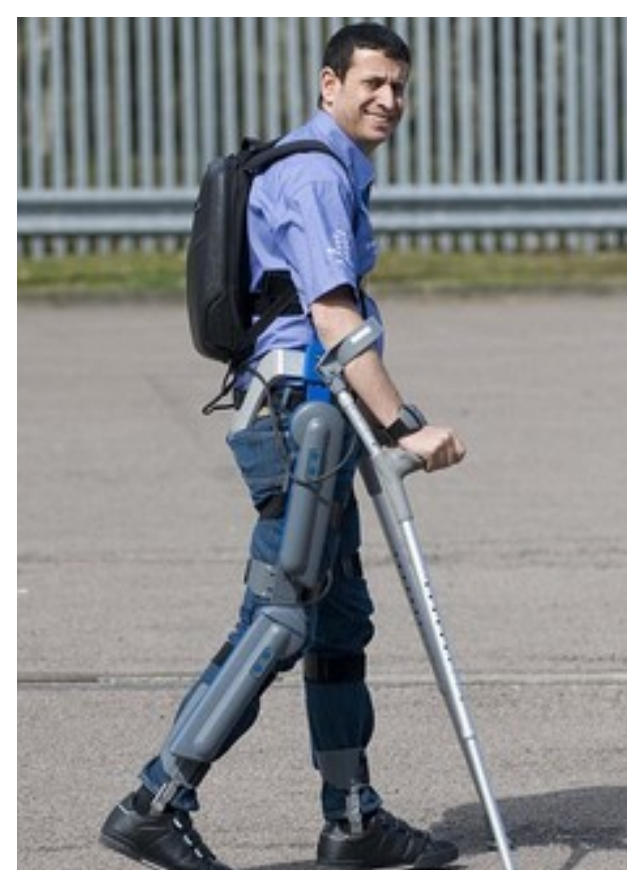

Figure 3-4: ReWalk robotic exoskeleton [123]

Further review of technology in this area has been completed by the author [80] and others. Pediatric systems are the focus in the next section.

\subsubsection{Current Pediatric Physical Therapy Robotic Systems}

Pediatric physical therapy systems are in some ways very similar to adult counterparts. They are not always just scaled-down versions, however. Treatment of children versus adults differs in other ways than size [18]. 
Notable differences include having more uncertain prognoses, needing multiple areas of rehabilitation, not having any experience of performing certain tasks before (e.g., they are learning to walk, not relearning), habits are evolving more quickly than in adults, and children are already dependent on many individuals. It can also be noted that there are not as many studies showing the efficacy of robotic-aided rehabilitation in children as with adults. While there is reason to believe that the technology will be helpful and some studies have proven this to be the case, it hasn't yet been established in a wide range of studies, not many studies with controls, nor for a wide range of robotic systems [76] [31].

There are some systems that exist or have been modified specifically for the neurorehabilitation of children. A few are noted in the paragraphs below.

The InMotion2 system has been used with children. In use with children with cerebral palsy $(\mathrm{CP})$, it was found that not only did the robot help bring about motor improvements, but it did so to a greater extent than studies with adults [30]. 


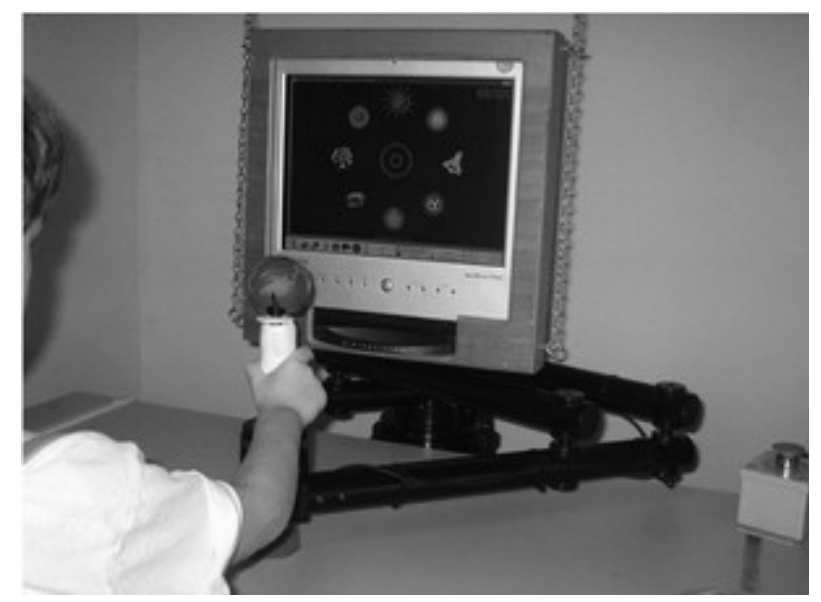

Figure 3-5: InMotion2 system in use with a child [30]

The New Jersey Institute of Technology Robot-Assisted Virtual Rehabilitation (NJIT-RAVR) system is comprised of algorithms and virtual reality equipment interfaced with a commercial Haptic Master system. It has been tested with two children with $\mathrm{CP}$ to successfully test viability of the platform [91].

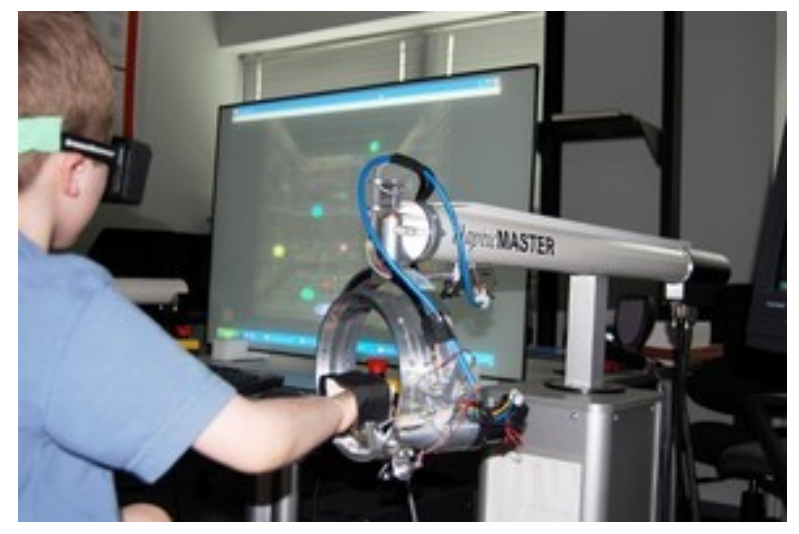

Figure 3-6: NJIT-RAVR [91] 
The Rutgers Ankle CP is designed to help train the ankle of children with the combined use of a robotic platform under the foot as the controller for a video game console [14].

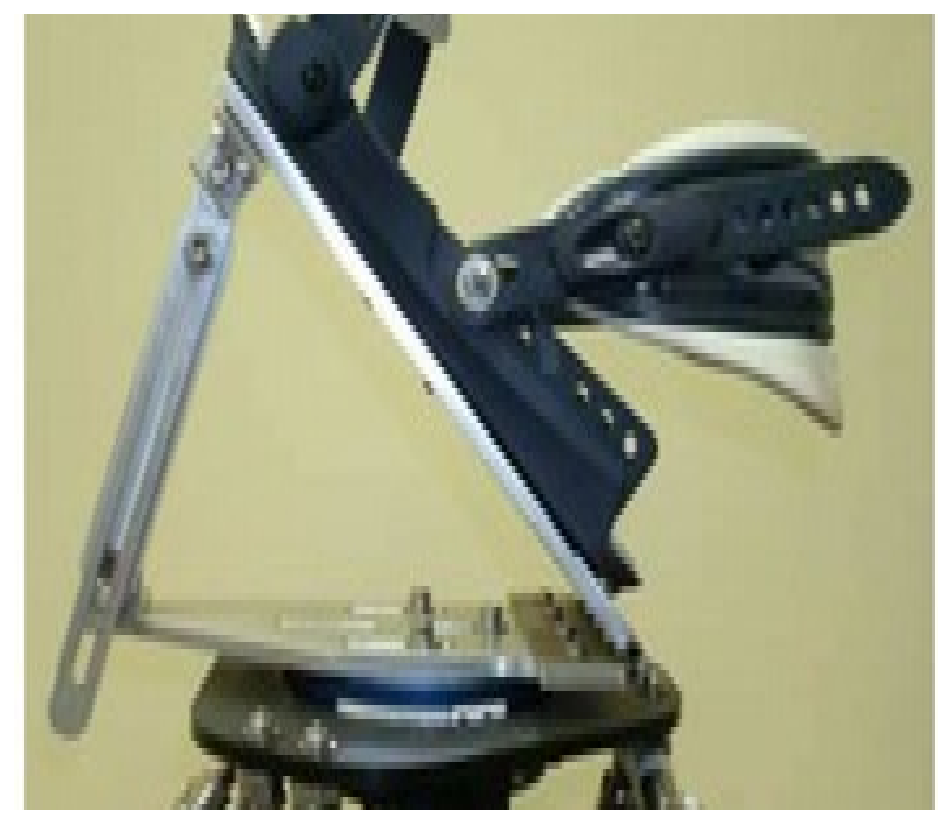

Figure 3-7: Rutgers Ankle CP [14]

A commercial gait-training system, the DGO Lokomat, has been used with pediatric patients. This European system has been shown to offer motor improvements for the children, though no control group was used [75] [8]. 


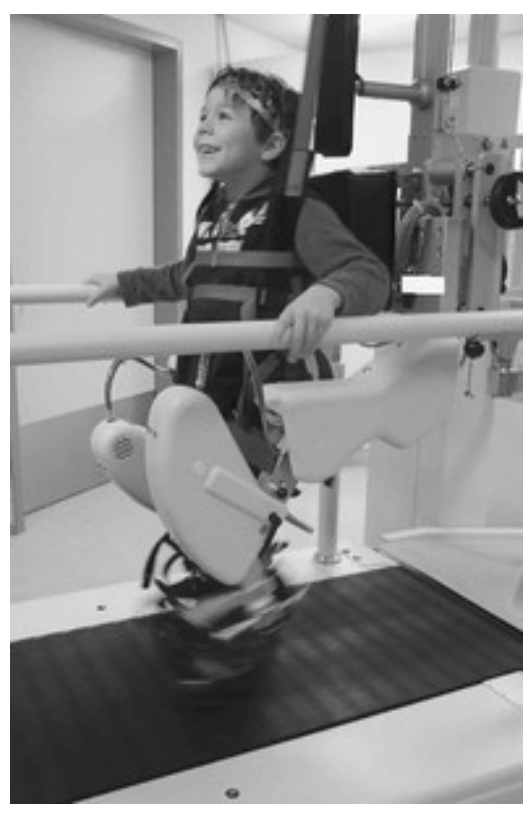

Figure 3-8: DGO Lokomat used for pediatric gait training [75]

The Gait Trainer GT I is another commercial system that has been used with children. This system is an exoskeleton that aids in the training of lower extremities. In a trial with children with $\mathrm{CP}$, it was shown to help produce improvement in motor abilities against a test group [105]. 


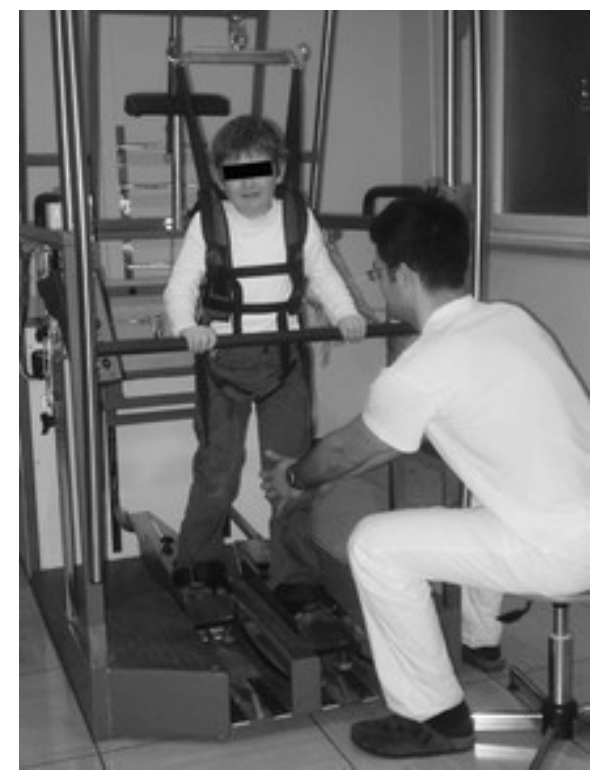

Figure 3-9: Gait Trainer GT I in pediatric use [105]

\subsection{Humanoid Robots}

Task modeling is an important part of physical therapy regardless of the patient's age. The task must be placed into context, made to seem important, and be performed in a realistic manner and speed [86]. Modeling this for someone with a physical impairment or difference can be very difficult for someone with normal motor function and physiology. Treated patients who have a similar difference or had a similar injury are highly effective in demonstrating to new patients [86], but it isn't feasible for most rehabilitation practices to have former patients available to model every new activity.

Therapy provided in natural context and environments is desired or even required by law [28] [51]. In practice, this is not typically implemented as 
intended. Instead, therapy in-home or in-school is often performed exactly as in an in-office setting even though the setting is natural.

Furthermore, therapy should continue past the short weekly half- or full-hour sessions with a therapist and be a continual part of everyday activities. Movements performed only for discrete time intervals once or twice a week have a much more limited result than those performed daily, if not multiple times a day. In some instances, patient compliance performing activities may be limited or nonexistent due to lack of motivation or confidence [104]. Determined by numerous circumstances, some patients require more visits from therapist and professionals than others [51].

Humanoid robots can provide some solutions to these problems by having an inexpensive tool to better model tasks in a manner that is realistic and achievable to individual patients, and to provide motivation and accountability to perform tasks when the therapist is not present. It can even be used as a teleoperated tool to provide quick and urgent consultations or to supplement home or office visits without the need for the patient or therapist to travel. Finally, humanoid robots can act as an intermediary between a human therapist and a child on the autism spectrum to achieve interaction and participation from the patient that may not be possible otherwise [101]. For these reasons, humanoid robots are of interest in physical therapy and some current models are noted below. 


\subsubsection{Partial Humanoids}

Depending upon the needs of the patient, a full humanoid robot may not be necessary. Only focusing on the parts that are affected or undergoing therapy can reduce system complexity and size in addition to making the robot less expensive and less intimidating.

Projects that are incomplete humanoid projects include the face, hands, arms and legs. In some ways, industrial robots can be considered mimics of human arms, but this review will focus on robots that can be used as anatomically-correct human models.

\subsubsection{Humanoid Faces}

Faces have been used on robots since their inception. However, making faces that are correct anatomically and physiology is a goal for numerous reasons including making both complex and simple social robots [5]. Hanson Robotics has numerous realistic robot face systems including one that looks like Albert Einstein and another called the Zeno R-50 [100]. Some expressions it makes are shown in Figure 3-10 below.

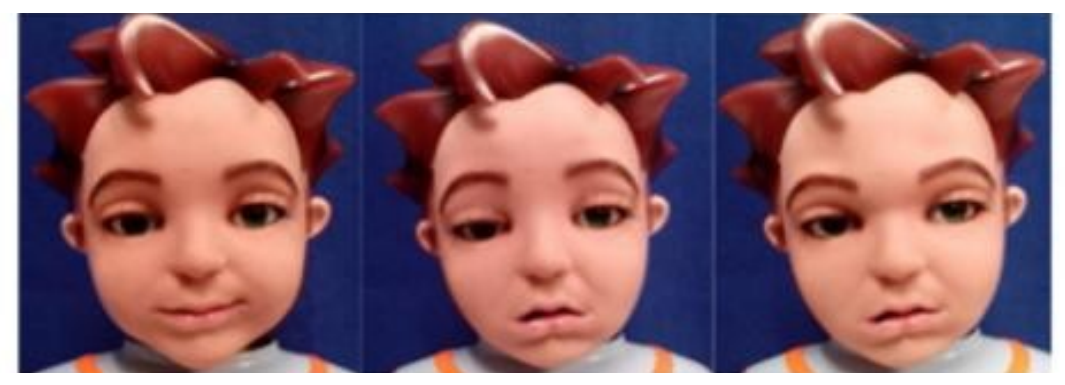

Figure 3-10: Zeno showing (from left to right) happy, sad and angry faces [100] 
A major aspect of the face is the eyes. These not only are a major part of expressions, but they have fast movements. Relating to the other aspect of this work, eye movements are a consideration when conducting eye exams and surgical procedures. Realistic robotic eyes within a face can provide a means for physicians to practice procedures similar to that performed on living humans when anesthetization is not preferred. One project from Italy attempts to do this using actuation inspired by human anatomy [20]. An image of this system is shown in Figure 3-11.

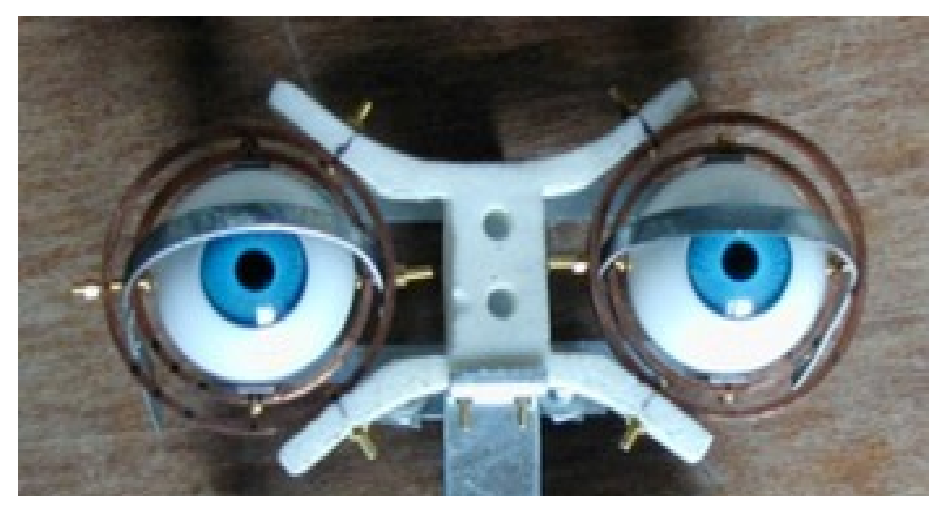

Figure 3-11: Robotic Eyes [20]

\subsubsection{Humanoid Arms and Hands}

Arms and hands are another area where work is being done to make robots that look like realistic human models. There are numerous hands that have been built over the years [35]. These are made to study human hand dexterity as with the MIT-Utah hand [77], serve as active prosthetics for amputees [23] or children born with differences in their physiology [124], and to simply mimic 
humans or be a research tool [120] [88]. Some examples of these are shown in the figures below. The last of these, the InMoov robot, not only has articulated arms and hands, but also includes the possibility to add torso and head/neck control as well.

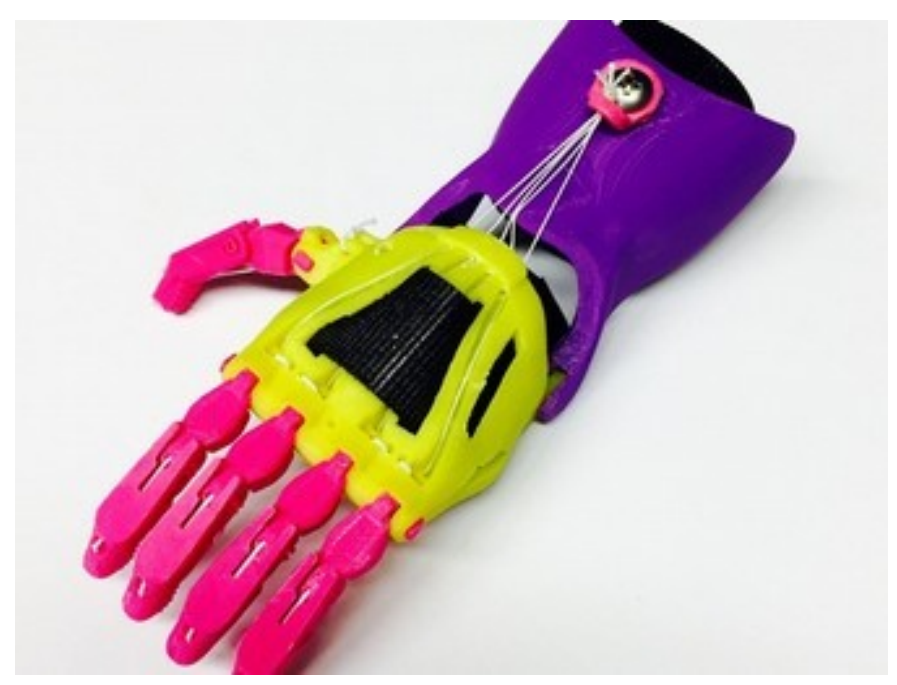

Figure 3-12: Cyborg Beast robotic hand [124] 


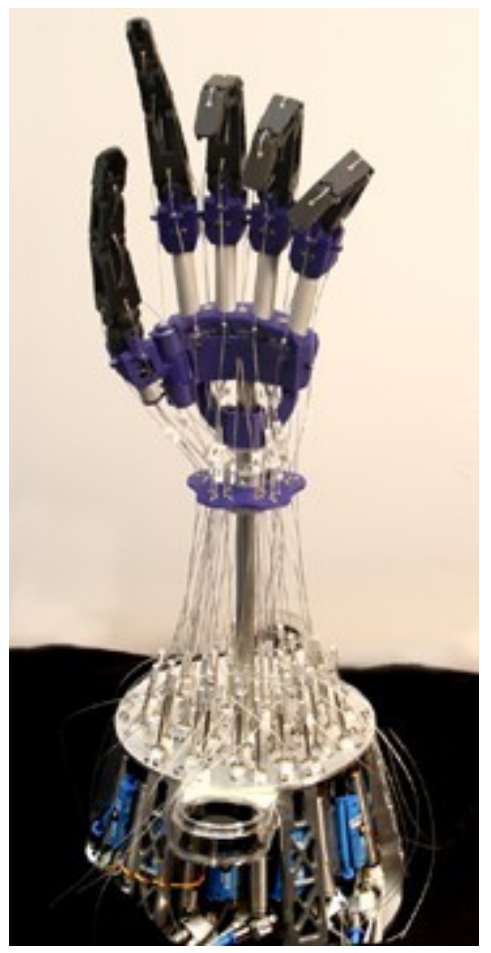

Figure 3-13: Anthropomorphic robotic hand [120]

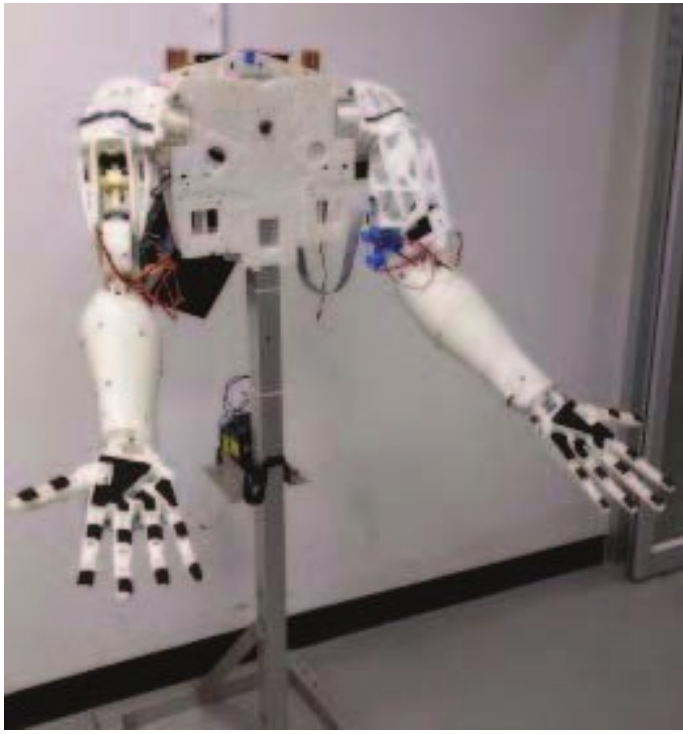

Figure 3-14: InMoov robotic arms and hands [88] 


\subsubsection{Complete Humanoids}

Humanoid robotics have always been of interest as can be seen by various depictions in science fiction and the many uses that they have [110]. For use in physical rehabilitation, sufficient articulation and realistically-proportioned limbs are necessary to properly model tasks. In the case of children, smaller models are also warranted. Though it is possible for the robots to be life-sized, this is not necessary. Smaller robots can demonstrate movements well and are easier to transport, less likely to cause injury to a child, and can be less expensive. Smaller robots also allow for some simplifications and may allow for older children or their parents to participate in their set-up so as to reduce some anxiety in their use [108].

There are some commercial humanoid systems on the market that allow users to program movements and interactions with the robot. Popular examples include the NAO [73] and Darwin [79] robots. The NAO, shown in Figure $3-15$, by Softbank Robotics is a popular system for educational institutions for programming motions and studying motion control or humanrobot interactions. It has $25-\mathrm{DOF}$, is nearly $600-\mathrm{mm}$ tall and costs thousands of dollars. The Darwin-OP by Trossen Robotics, shown in Figure 3-16, is a system that is slightly smaller than the NAO and has only 20-DOF, but costs hundreds of dollars instead of thousands. 


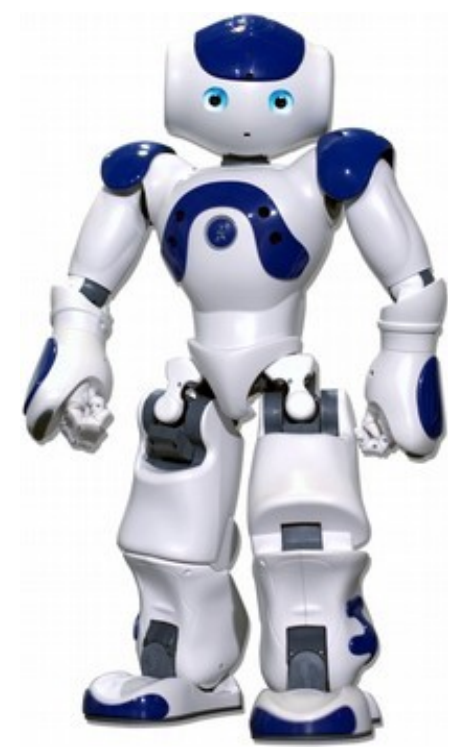

Figure 3-15: NAO humanoid robot [73]

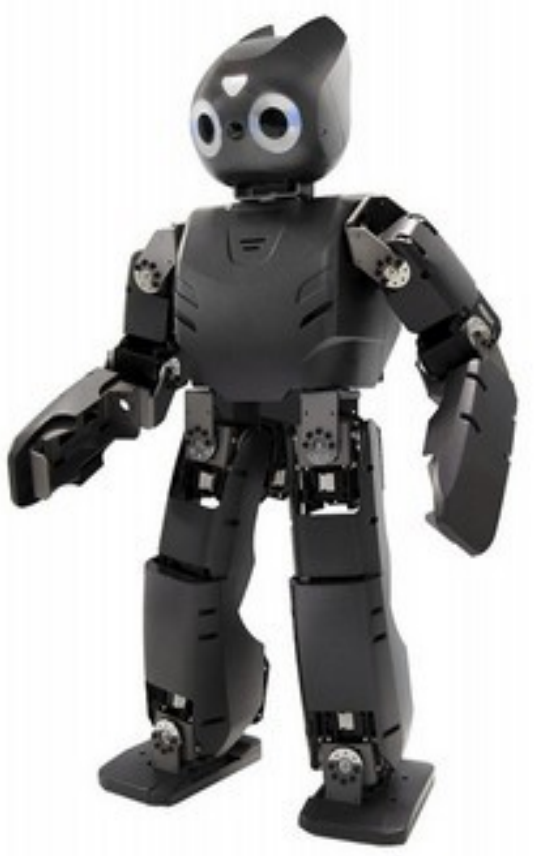

Figure 3-16: Darwin humanoid robot [79] 
Open-source and custom-designed systems allow for physical flexibility in addition to software programming. Nearly every aspect of these robots can be customized to meet desired specifications including size, strength, speed and cost. A popular system is the Poppy Project [63]. Though it has many variants, the original, shown in Figure 3-17, is $25-\mathrm{DOF}$ and nearly $850-\mathrm{mm}$ high and can be used as an open-source alternative to the NAO.

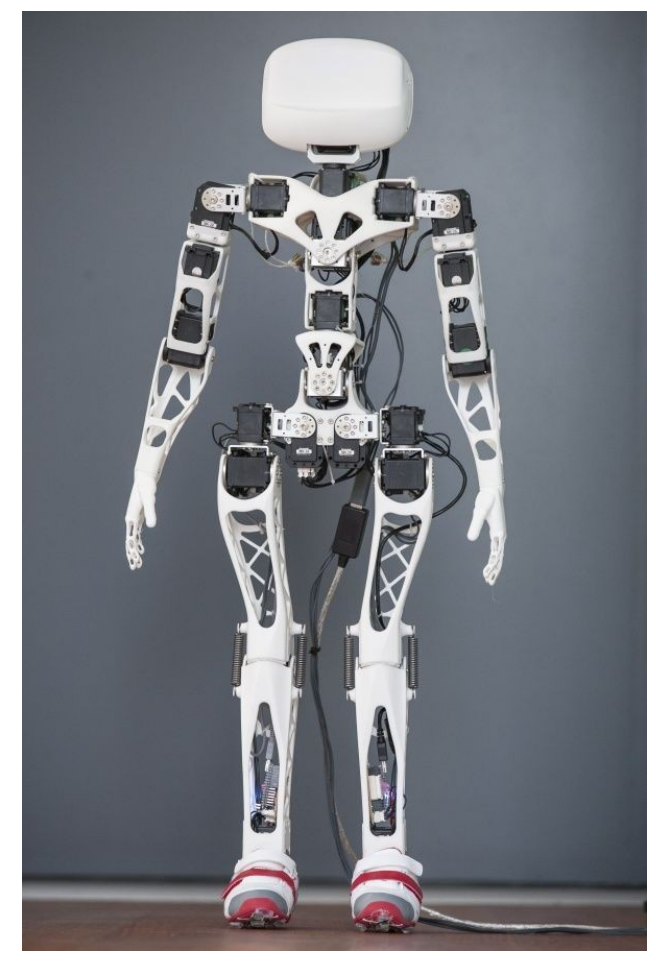

Figure 3-17: Poppy Project humanoid robot [63]

The next chapters develop technology based on the background provided in the previous two chapters in order to meet the goals and objectives of this work. 


\section{THEORETICAL BACKGROUND}

This chapter reviews and develops theoretical issues that pertain equally to both types of medical robotics discussed in this dissertation. This includes issues with remote operation as well as the theory of control systems that are used later in this work. An overview of what will be done in each area is provided followed by notes about use in medical robotics. Finally, different control systems are surveyed and evaluated for use in the scope of this work.

\subsection{Teleoperation}

Teleoperation is a large component of successful operation of the robotic system proposed in this work. While it is certainly possible and may be convenient for a physician to control the robotic slit-lamp from the same room, allowing the control of this device over a long distance provides a greater range of use and benefits. After reviewing general research in all forms of teleoperation of robots, the following sections discuss specific issues encountered in both tele-examination and tele-surgery.

Synchronous teleoperation has been tested for ocular procedures since at least 1997 [6]. Time delays are the biggest obstacle when controlling a robot real-time from a great distance. A contemporary system offers the simple

solution to time delays by simply adjusting the scale of the tremor and thereby 
smoothing the reactions [83]. It was noted that scaling too far slows the ability to complete the procedure quickly, so it is necessary to determine an optimal point to balance the speed of the manipulator with the time delay.

\subsubsection{Tele-examination}

There is a growing trend toward telemedicine in practice, with a current focus on tele-examinations and consultations. In ophthalmic applications, most current teleophthalmology practices are limited to consultation. While both synchronous teleoperation and store-and-forward models can be followed, only the later is currently practiced [60]. This involves manually taking general eye exam images as well as images of any features of interest and sending the information to a remote location for interpretation and diagnosis.

While most devices mentioned in a previous chapter focus solely on performing eye surgeries, eye examinations are an important routine step that must first be performed. This involves a synchronous teleoperation model where everyone involved in both the physician and patient locations are connected with real-time communication abilities.

Prior to this work, only one robot has been created with the intention of fully performing eye exams from a remote location in real-time [21]. This device, built and tested in Thailand, is able to give a remote physician the ability to perform a full eye examination with some help from an aide working with the patient. A slit-lamp, the tool of choice for eye examinations as described 
previously, is fitted with motors to allow for computer control of several major functions as shown in Figure 4-1. While traditional use of a slit-lamp requires adjustments of several knobs to alter the focus while performing an eye exam, the group devised an auto-focus algorithm to capture clear images of the eye. Due to concerns about bandwidth and delays, only select images are sent to the physician and text-based communication is used between the two locations. While the initial trials of teleophthalmology were less accurate and slower than traditional in-person use of a slit-lamp, the research group expected that further studies would show improvements as the physicians learned how to best utilize this technology.

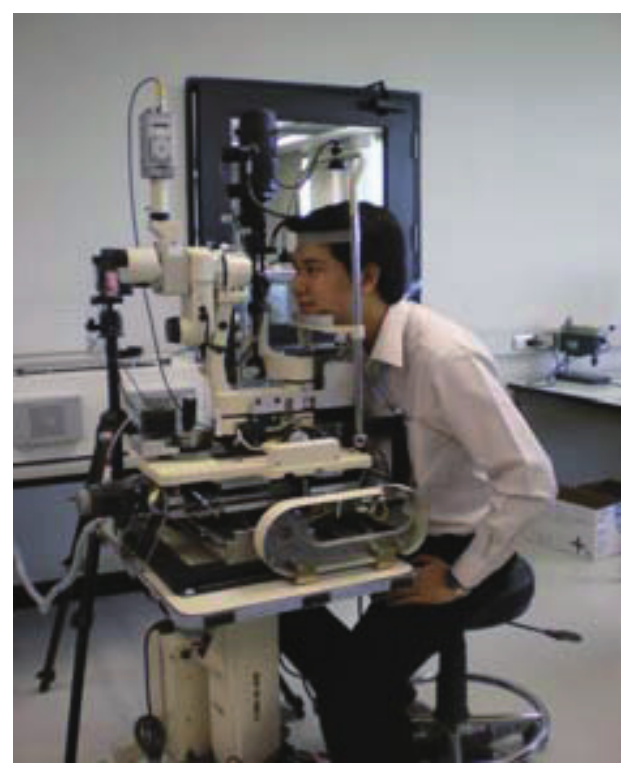

Figure 4-1: Robotic slit-lamp for teleophthalmology developed in Thailand [21] 


\subsubsection{Telesurgery}

Telesurgery is a little more complicated than simple exams because of the risks of harm from surgical tools in addition to possible danger of the patient unexpectedly getting in the way of the robotic mechanisms while in motion. There have been some cases of remote robotic surgery being performed [69], but it isn't currently a typical arrangement.

Especially in ophthalmic procedures, time delays can cause huge issues. A surgical tool may move into a harmful position before the ophthalmologist notices and can alter the motion or stop the laser, causing damage. Not realizing that a motion is in progress, but subject to large latency, a movement or action may be requested by the doctor that is already in progress and an overshoot in the desired position or action occurs. There is some work being done to develop an algorithm to determine when the human operator should be overridden and also when to employ automated subroutines [7].

For research purposes, open-source alternatives are interesting because they provide a means for multiple institutions to work on the same platforms and share information and ideas. This is not possible on commercial platforms since components, whether hardware or software, tend to be proprietary and are not easily manipulated to test alterations. One open-source project uses retired da Vinci systems and is developing a controller by parts that is run via an open-source Linux computer [53]. 


\subsubsection{Teletherapy}

Performing various types of therapy remotely lies somewhere between telexaminations and telesurgery in risks and complexities. Depending upon the style of the therapy robot, it may or may not require physical contact with the patient to perform. Examples of systems that require physical contact include the InMotion system and exoskeletons such as ReWalk. A patient may have more ability to discontinue contact and thus the operation of a system such as InMotion, but physical contact is still something to be considered and severely disabled patients should have someone, even if not medically trained, present. An exoskeleton provides more invasive contact and can not easily nor quickly be removed, especially by an impaired patient. These devices require much more robust safety measures similar to that used in telesurgery.

Robotic therapy systems that do not require direct contact are those used for modeling behaviors as proposed previously in this work. There is still reason to consider human safety, however. The patient should be supervised to check for proper mimicry and participation, but also as a precaution against any usual concerns such as poor balance or the inability to stand unaided. There are also some risks of injury should the patient or caregiver touch the robot during motion and be hit or pinched by moving parts. Decreasing robot size as well as increasing joint compliance are two major methods for passively increasing robot safety in these instances. 
In addition to considering how safe a robot is around a patient, the programming and control of the robot is another area of concern. Therapists will be required to program these robots and few are programming experts. There are three major methods of programming including kinesthetic (moving the actual robot by hand to points it should be), remote control via a physical model or joystick, and control and programming via a software interface (either utilizing a specialized program created to capture motions virtually or by numerically programming points). All of these have advantages and drawbacks depending upon the situation and user preferences [33]. Determining the best method for a given therapy robot is a necessary task to complete the project. The therapist must be able to comfortably program and adapt the robot to the needs of individual clients in order to gain the maximum benefit.

\subsection{Control Systems}

Control systems in robotics serve to control the movements to follow a desired outcome. This is a key design aspect in robotic technologies and a lot of work as been done in this particular field. Most of the work is theoretical and applied on idealized or simplified models. When a control system is actually implemented, most systems utilize expensive control hardware and peripherals to complete the task. Others are limited and do not perform exactly as the original control design intended due to hardware limitations. Recent trends are 
allowing for more complex control methods with lower equipment costs as processors become faster and cheaper [25]. This enables the consideration of using embedded systems to perform control methods that only large manufacturing plants could consider before.

The market for some control systems is changing. The Maker Movement [27] is one catalyst that has changed how robotic technology is implemented. Globalization as well has led to the desire to use standardized equipment and cut costs, so that systems can be affordable and obtainable on the world market. There are now numerous low-cost, yet versatile hardware options that can be used as powerful robotic control systems. This work will focus on these technologies. Specific examples include the Raspberry Pi, BeagleBone and Arduino. New low-cost PLCs, or used low-cost systems, can also be used as well, though the availability to alter the control algorithms for these devices are limited. Other technologies that are not explored in detail here include mobile devices such as tablet and smartphones, PCs with USB GPIO, small microcontrollers such as the PIC, DSP or motion-control microcontollers, and hardware-in-the-loop systems.

There has been little research in literature on implementing control systems for Maker Movement devices. While it is easy to find numerous projects using inexpensive hardware, they are usually simply plugged together and programmed to perform a task. In some ways, technical engineering analyses counter the ideals of the Maker Movement; Many see it as a way to open up 
the ability to make technical objects to everyone without the need for highly specialized knowledge. In reality, research in this area would benefit all Makers; though not all may choose to delve into the foundational literature and fully understand technical nuances. In any case, research and improvements can be made available through open-source instructions and code to be utilized by those who find use in their application. This benefits hobbyists, educators and students, as well as the entrepreneurs and small businesses that are using the low-cost devices in small-scale manufacturing and medical equipment. It is possible that in the future, these inexpensive devices may become capable and robust enough to be of serious consideration for market-use in moderate to large-scale manufacturing facilities and the medical device industry.

\subsection{Control Systems in Medical Robotics}

The control systems in medical robotics do not actually differ that greatly from those used in industrial systems. Many may think that medical systems require greater accuracy or more features; in reality industry does, and has, been demanding very similar specifications. One must only consider how intricate many modern electrical devices have become to see how assembly of even mundane objects requires high precision and accuracy. In addition, the motions of many medical and manufacturing robotic systems correlate: cleaning and painting, physical rehabilitation repetitive pick-and-place and manufacturing pick-and-place, and surgical site targeting and part targeting. 
The materials the robotic devices are made out of and the exact implementation of the end-effector are the only real differences between industrial and medical robotics. Medical robotics typically require materials that are easy to sanitize. There should be some degree of tolerance for fluids and UV lights as the cleaning process is critical. Industrial robots should be made from materials that can withstand the chemicals and abrasions of materials that may be in their environment. This difference has some influence on the controller as the weight and dynamic characteristics may be affected, but usually it is not significant. Therefore, techniques used in manufacturing can be used for medical robot control systems.

It is also worthwhile to keep an open-mind in the use of control methods from other industries outside of robotics to be applied to robotics problems. Numerous instances can be found of control methods from one discipline being used in another. In many cases, one can find great similarities between models and equations used in one type of system to another. Electrical circuits and hydraulic systems are sometimes modeled with the exact same equations using only different symbols for the variables, for example. Even with different equations, control systems can provide new solutions or just new insights in multidisciplinary settings. 


\subsection{Control Methods}

There are numerous control methods found in engineering literature. This work will not attempt to list every type. It is helpful to try to classify them in order to consider major types and examples. This classification is somewhat arbitrary and is not meant to imply that this is the only way to consider these control techniques. Broadly, the following sections will briefly survey classical control, hardware control, software control, and control techniques that don't fit well into one of the previous categories [1].

\subsubsection{Classical Controllers}

Classical controllers are ones that are found in nearly every engineering textbook on control theory and are in use in innumerable applications. They work for linear systems and can be the most efficient solutions in many instances. Examples include hysteresis and PID controllers.

The simplest types of classical control are the hysteresis or on/off methods. These can be used in both continuous or discrete systems [3]. This type of control typically works between two different boundaries with the input being one of two states. Figure 4-2 shows a block diagram. Advantages to this type of controller is that it is fast and stable. However, it can lead to undesirable or variable frequency changes which can cause premature failure or electromagnetic interference [16], though there are numerous altered hysteresis controllers that address these shortcomings. There are different 
types of hysteresis controllers including bang-bang and deadbeat controllers. This type of controller is most commonly used in HVAC systems or to stabilize the output of filters in electrical circuits.

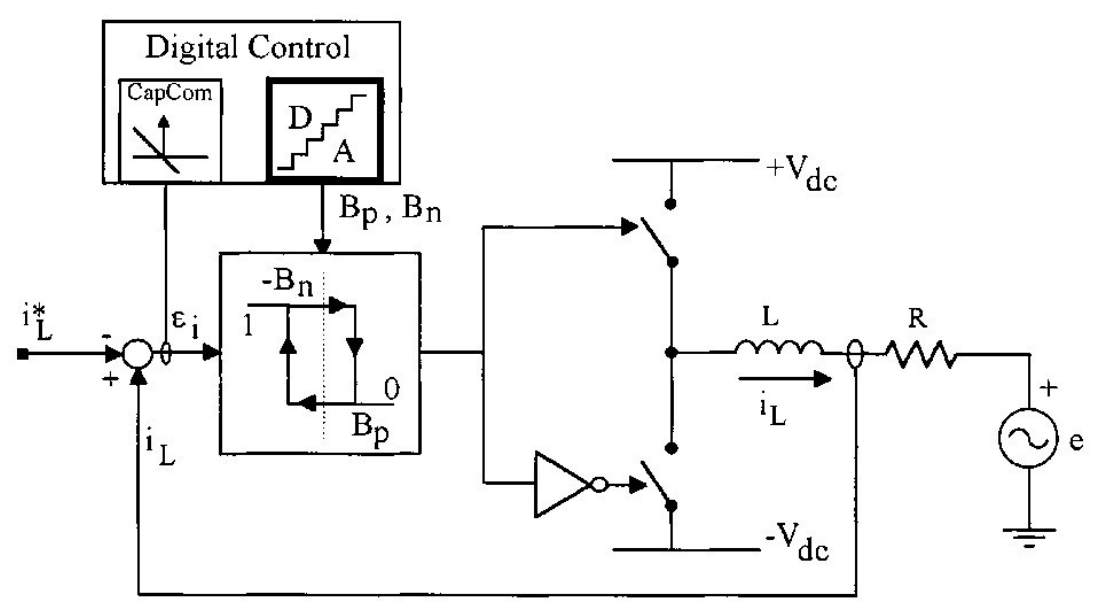

Figure 4-2: Schematic of a simple hysteresis control system [3]

PID controllers and their permutations are the classical continuous improved solution to the hysteresis controllers. In general, these controllers are tuned for a particular linear system. When they are properly tuned, they can work well and efficiently. However, PID controllers quickly become less effective when the system operates outside of specifications for which it was tuned. Tuning and even auto-tuning can be cumbersome and intrusive [1].

PID controllers produce an error value that is calculated as the difference between the desired reference input and measured plant output variable. The controller uses the output calculated from a proportional $(P)$ term and usually 
an integral (I) and/or derivative (D) term to attempt to correct the plant output to reach an error of zero. Depending upon the terms used, the controller may be referred to as a P, PI, PD or PID controller. A schematic of this type of controller is shown in Figure 4-3 below.

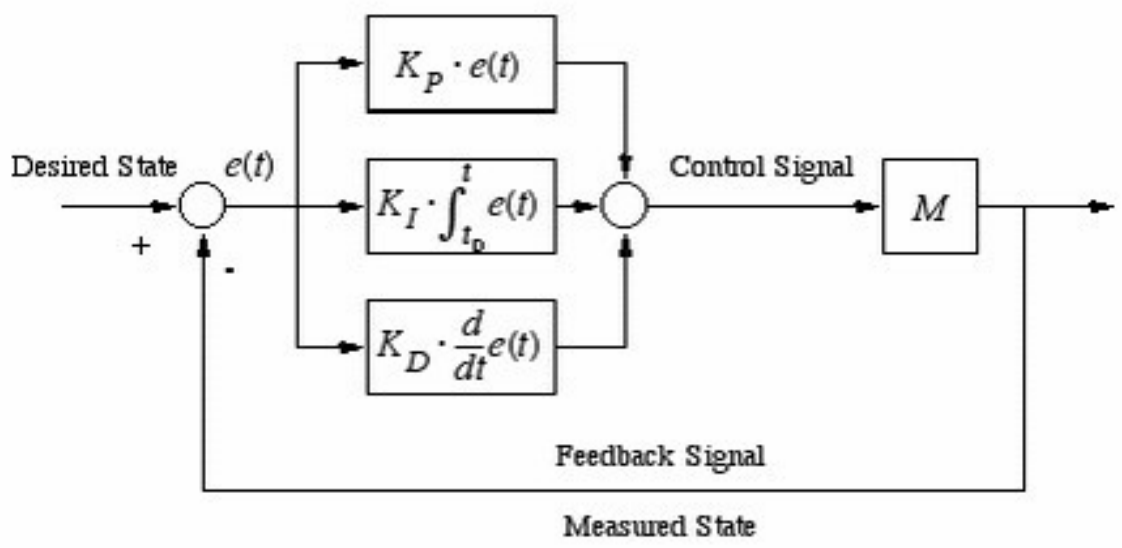

Figure 4-3: Schematic of a basic PID controller

PID controllers are extremely common in industrial systems. This type of controller and all of the many hybrid and adapted versions can be implemented in many ways and they can be found in both technical literature and in control system hardware specifications. They can be programmed in simulation, using high-level programming languages on a variety of computing hardware, or built into a chip.

Another way to implement PID control methodologies is with Programmable Logic Controllers (PLCs). These are modular systems that are extremely robust, but many are limited in the complexity of programs they can handle by 
internal memory and the software or hardware available in which to program them. Each brand of PLC also has its own structures and instruction sets, so programs can not be used from one type of device to another [12]. Still, some PLCs such as the Allen Bradley SLC 5/02 have built-in PID algorithms for closed-loop control that can be utilized.

\subsubsection{Hardware Controllers}

These types of controllers are based on control theories that originated with hardware controls, but are a step away from classical control in that they delve into nonlinear, robust and optimal scenarios [1]. Examples of this type of control include sliding mode control and predictive control.

Sliding mode control is one type of controller that differs from classical control in that it is non-linear. The feedback control changes based upon the state of the system and the position it assumes on a selected sliding surface. One must adjust the feedback so that the system stays on the selected surface at all times. The control used can be simple or complex and combine features so that the controller is extremely robust.

Predictive control comes in several varieties. Some examples are hysteresis-based predictive control, trajectory-based predictive control, and a type of deadbeat control [25].

Another type of predictive control is Model Predictive Control (MPC). This methodology utilizes a process model to predict the future response of the 
plant and optimize future behavior at each control interval [90]. Numerous variations on this type of control exist in the literature including finite control to lower the complexity [25] and explicit models to solve for interesting conditions off-line and simplify on-line calculations [2]. They are also being applied to increasing numbers of applications in more diverse fields as well. One reason for the pervasiveness in literature is because MPC controllers are commercially available [90] [25]. Still, most MPC methods have drawbacks that include a lack of internal (nominal) stability, and lack of dynamic optimization [90]. Tuning is also a problem and can only be done for certain models and may not result in a stable controller even with the perfect model [90]. There are many works on improving these controllers, but it is one case where the improved algorithms are not yet fully implemented in commercial controls.

\subsubsection{Software Controllers}

These types of controllers are purely software-based. They are only possible because of digital computers [1]. These include Fuzzy Logic and Neural Networks.

Fuzzy logic controllers are programmed by rule sets that provide output reactions for given inputs. Several rule sets are programmed for any given controller that is developed. These sets are then used to make a decision for

the output of the entire system given the state of the inputs. This type of controller does not require a lot of processing power and so can be made low- 
cost, even for unknown or inexact models. Drawbacks are that the outputs must be tested for input states and so validation and tuning of the controller can only be done by trial-and-error.

Neural networks attempt to model 'thinking' behavior. They are trained by giving them inputs and programming the appropriate output. After training, they use this as a guideline to determine what to output based on examples that were given in their training. The algorithm in a neural network can be simple to complex, so the processing power varies. Part of the design in this controller is having a sufficient algorithm without being unnecessarily memory and process intensive.

\subsubsection{Other Controllers}

Some controllers don't fit neatly into one of the above classifications because they are a hybrid of control techniques. Examples of this include Hardware-in-the-Loop controllers and Fuzzy-PID controllers. There are also other controllers that are more advanced or specialized and will not be discussed in the scope of this work.

Hardware-in-the-Loop controllers are not exactly a new control methodology, but real time simulations that have actual devices embedded in the loop instead of models for these components. This allows for a controller simulation to fully take into account limitations and actual signals from sensors and such rather than depending upon an idealized model [12]. This can speed 
up development and validation of embedded controllers. Simulation tools such as LabView are frequently used in this type of controller.

Hybrid control techniques are numerous and found throughout control literature of nearly every field. Proposed and implemented hybrid control systems can span and combine any control methodologies. PID, MPC, and Fuzzy Logic controllers seem to especially lend themselves to be utilized in hybrid systems. In the case of PID controllers, they are the classical benchmark and are nearly universal. They are also found throughout industry and so have practical importance that can benefit from adaptations. MPC controllers are also used in hybrid systems because they are commercialized and can be better adapted to specific instances of interest. Fuzzy Logic is used in hybrid systems because it is a relatively quick and low-cost adaptation that can provide easily understood changes to the controller with which it is combined. 


\section{OPHTHALMIC PROTOTYPES DESIGN AND CONSTRUCTION}

This project involved building a physical model to serve as a proof-ofconcept and as an initial testing platform. This chapter provides details on the two iterations of prototypes that were produced. For ease in reference, the project was dubbed OphthBot and first prototype is usually referred to as OphthBot 1 and the second is referred to as OphthBot 2.

The robotic slit-lamp presented here served as the proof-of-concept for this technology. This low-cost system was developed to help obtain further sponsorship and lead the way toward improved prototypes for medical testing. For the purpose of this work, the goal was to make the robot as accurate as manual procedures currently performed. Procedures were to be limited to those done with a standard slit-lamp and hand-held lenses, though the addition of a laser integrated in the slit-lamp was a key consideration as well. No instruments were to be inserted into the eye. All testing during the time this work was completed was done on anatomically-correct plastic eye models and not on human nor animal tissue.

The robotic slit-lamp was to be designed to work with a medical aide present with the patient. This medical aide helps set-up the patient and makes gross adjustments to the slit-lamp. The medically-trained individual also helps the patient during procedures and is available to assist the patient in the event 
of any technical fault. Major components of the complete technical system are shown in Figure 5-1 below.

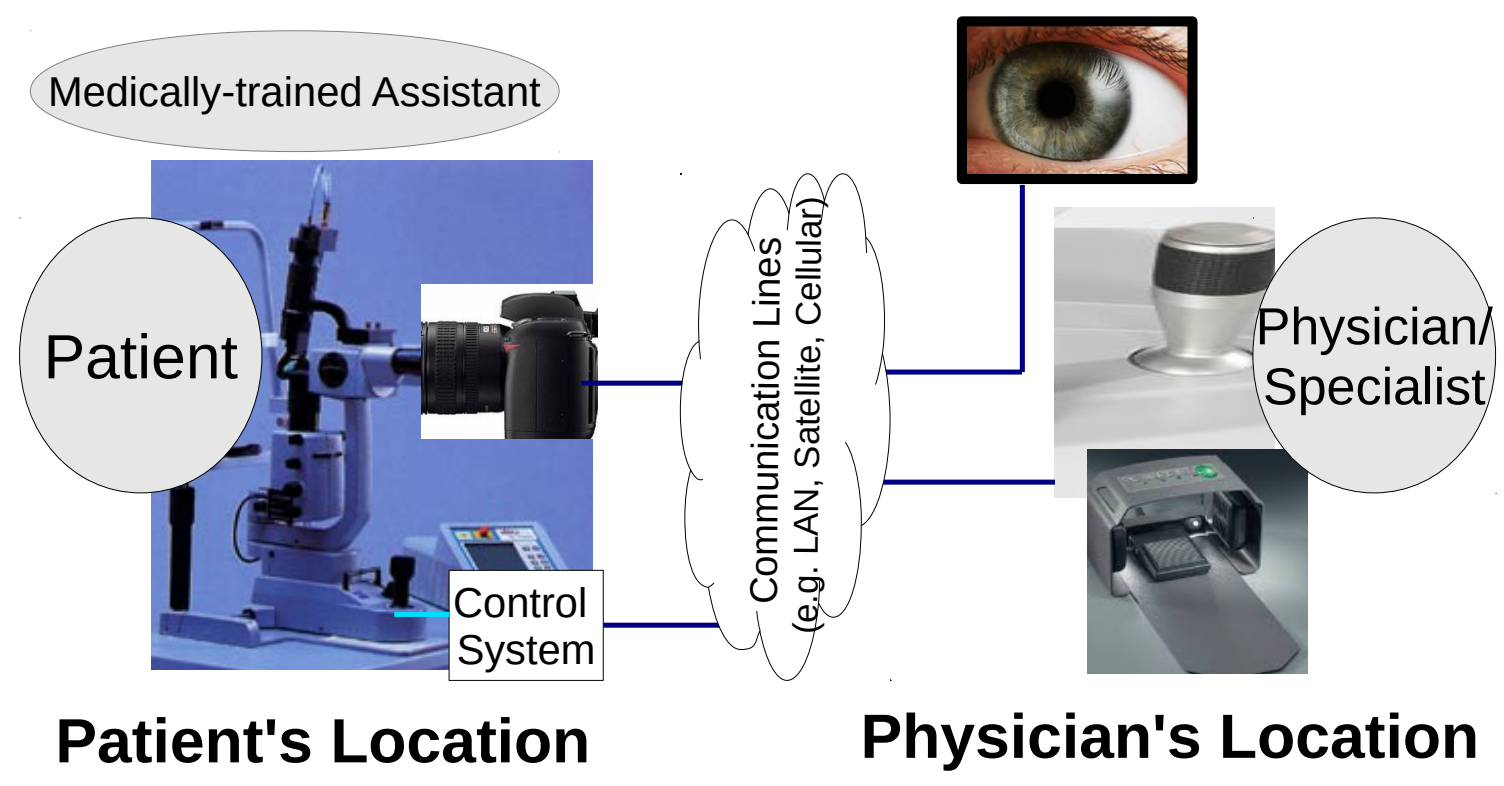

Figure 5-1: Major system components of OphthBot

For the rest of this chapter, sections 5.1 through 5.5 will discuss the first prototype, OphthBot 1, in detail. Section 5.6 discusses the major changes made of the second iteration, OphthBot 2.

\subsection{Automation of Base Slit-Lamp}

The prototypes were based was a standard slit-lamp. A vintage Neitz SL/W-J shown in Figure 5-2 was utilized. The slit-lamp is similar to Zeiss-type units and was also sold by Wesley-Jessen, but is no longer in production. It 
includes standard features such as adjustable light width, height, intensity and three light filter options. It does not include a means to disassociate the focal point of the microscope and lamp nor any digital features found on many current models. Moreover, no laser adapter, specialized accessories for cameras, or other tools are available as the unit has not been in production for many years.

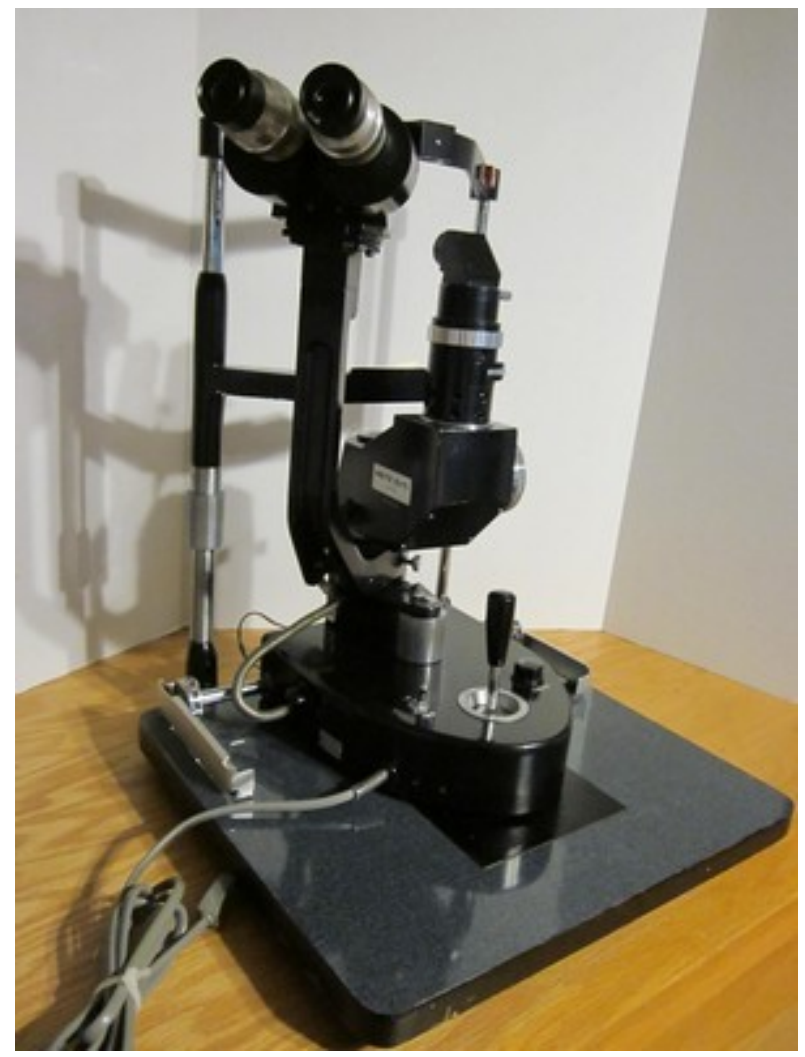

Figure 5-2: Base slit-lamp used for the prototype

In order to standardize nomenclature used in this work, the base refers to the base of the slit-lamp that moves on the table. The knobs are named by the 
feature in which they alter, for instance the light width knob controls the width of the light that shines on the patient's eye. The arms refer to the swinging parts mounted near the rear of the slit-lamp base. The microscope-binocular arm is the lower one which allows the eyepiece portion to change angles, while the lamp arm is the upper rotational part that allows the light angle to be changed. These parts are labeled in Figure 5-3 below.

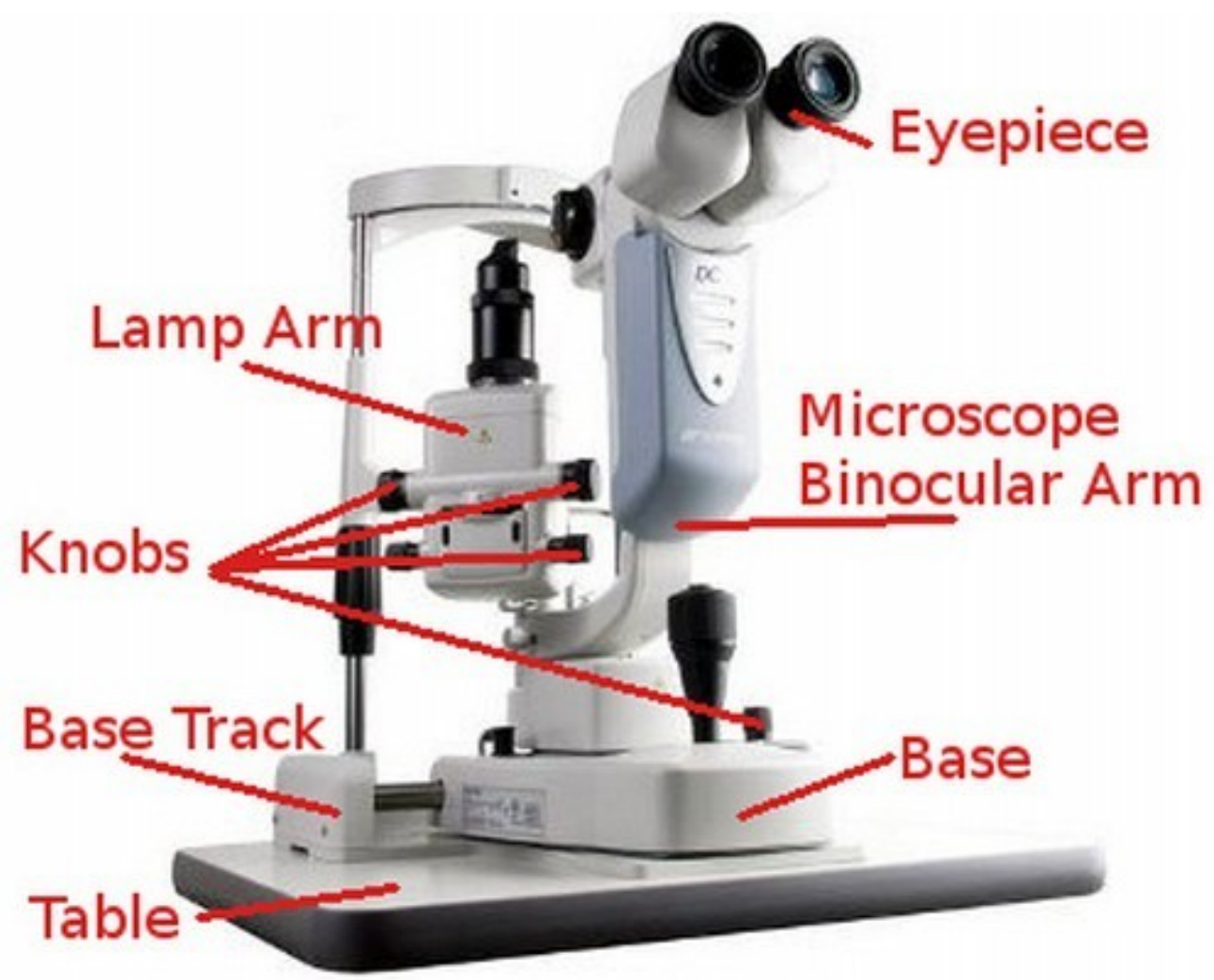

Figure 5-3: Names of parts referred to on a slit-lamp 


\subsubsection{Actuator Selection}

The first step was to determine the actuation method. Due to the need to control the position and accuracy rather than velocity, stepper motors and servo motors were considered.

Stepper motors are available in a wide range of sizes and load ratings, starting at a low-moderate price range. They are a good choice due to their options of either rotational or linear (usually via a screw mechanism) motion outputs. They do require more sophisticated controllers, however, as they have multiple control lines that must be activated in specific sequences. This adds hardware along with the associated integration and costs. This is becoming less of a concern as stepper motors are being used by Maker Movement enthusiast to produce machines such as desktop 3D printers. Availability and costs of commercial stepper controllers are rapidly decreasing.

Servo motors are also available in range of sizes and load ratings, however the price range varies significantly. Servos tend to fall into hobby and professional categories with costs varying accordingly. As with stepper motors, the Maker Movement has increased interest in easy-to-use devices for automation projects and the range and availability of very inexpensive servos have thus increased. High-torque hobby servos intended for robotic applications are available at very affordable costs and are easy to use. In addition, control hardware for these servos are also easy to find and are well documented. Finally, many servos are position-controlled and offer the 
advantage of built-in position feedback and do not require additional wiring, hardware and programming to use this feature.

Though stepper motors may offer better control in some situations, servos were selected for the initial prototype due to their ease of implementation. This was deemed the most important consideration given the short time-frame in which to complete OphthBot 1 . Servos offering sufficient torque and accuracy had to be selected so that the prototype would meet the criteria developed.

It was determined through experimentation that the torque required to rotate each of the arms and knobs as well as the linear force required to move base of the slit-lamp varied notably between trials. It was decided to size the actuators for the largest of these possible forces to ensure that they could handle all variability in the forces during operation. The maximum amount of torque needed to rotate the knobs and arms was found to be approximately 8$\mathrm{kg}-\mathrm{cm}$. This was too high for a typical hobby servo such as a Futaba S3003, so stronger servos were sought.

The servos selected for this project were the Turnigy TGY-SM-8168R oversized robotic servo for all of the movements that required a full rotation actuator and the Turnigy TGY-S810 oversized digital robotic servo for the components that could be driven with less than $180^{\circ}$ of rotation actuation. Both of these servos are shown in Figure 5-4 below. 

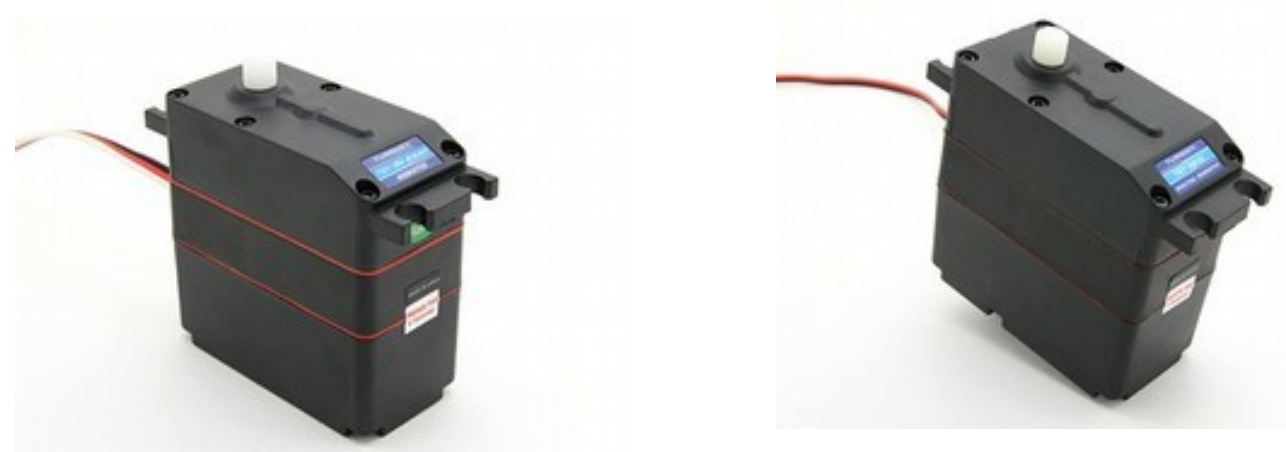

Figure 5-4: Turnigy TGY-SM-8168R (left) and TGY-S810 (right)

The addition of sensors for position feedback of the rotational and linear motions caused by the continuous rotation servos was also a consideration. The internal feedback of these servos are for speed, not position. However, the goal was the keep the project as simple as possible in order to facilitate quick prototyping and allow for easy changes later as the project progressed into later iterations. It was thus decided to not implement additional position sensors outside of those included inside the servos for the OpthBot 1 iteration. Instead, position was calculated via software and occasionally calibrated.

The use of digital position-controlled servos for most of the actuation alleviated the need to include encoders, though additional sensors would allow for verification and automatic start-up positioning. In the initial prototype, additional sensors were not addressed as all motion was manually controlled and also very limited. As a safety measure, limit switches for all actuated ranges are recommended in order to ensure that mechanical limits are not exceeded and damage to the robot and patient are avoided. 


\subsubsection{Actuator Implementation}

Attaching the actuators for OphthBot 1 involved primarily the goals of allowing for accurate and precise actuation of the slit-lamp joints. In addition, it was desired to allow the actuators to be easily removed in order to allow the slit-lamp to function in a fully manual mode. Finally, making the robotic retrofit as universal as possible was a tertiary goal as it would help the future development of nearly universal retrofit kits. Retrofit kits are of interest because it reduces the costs of the robot if medical facilities can use equipment they already have, or purchase used slit-lamps.

There were three methods in which the actuators were implemented that included friction-fit torque transfer, belt-drives and direct-drives. As labeled in Figure 5-5, the rotational lamp arm (2), binocular-microscope arm (3), and limited-rotation knobs (1) were controlled via the digital servos which were friction-fit to the components. A belt system was used with a continuous rotation servo for the slit-lamp height adjustment (5). A continuous rotation servo was friction-fit to the chin rest adjustment as well (4). Finally, directlydriven wheels were used to control the base movements of the slit-lamp with continuous rotation servos (6) and (7). 


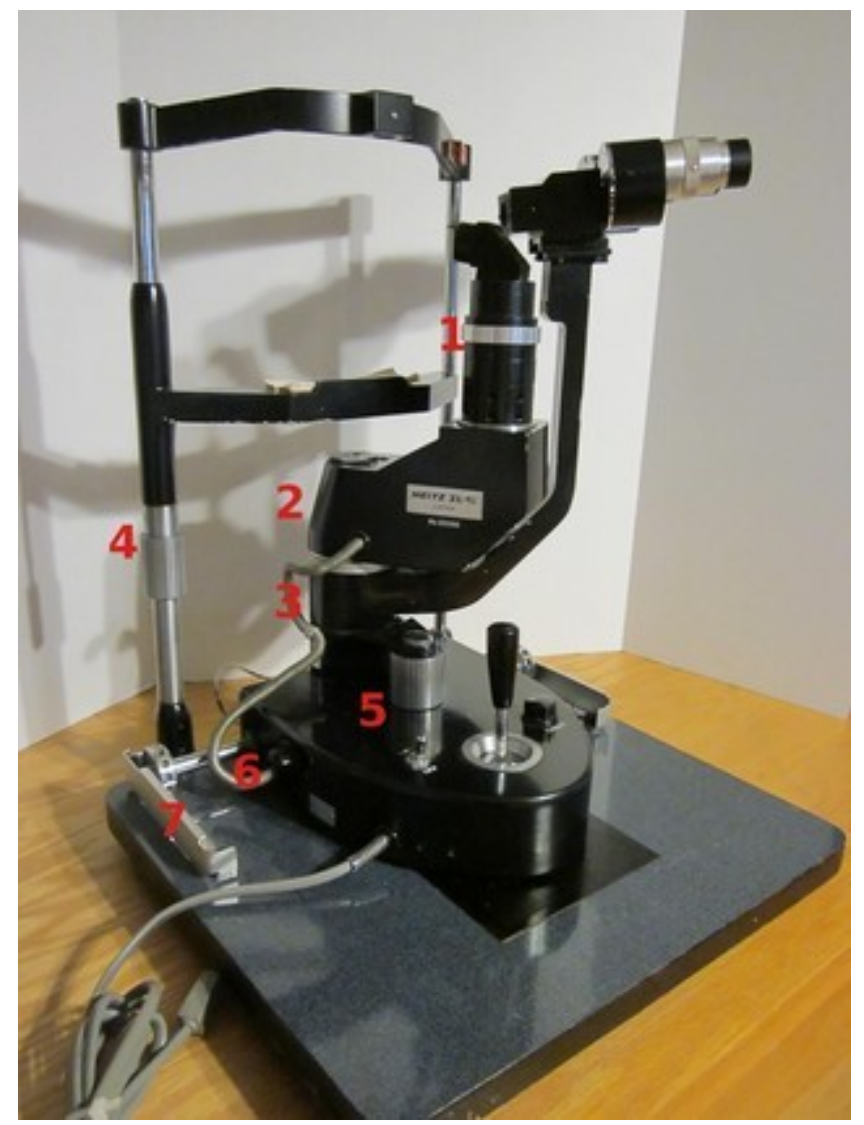

Figure 5-5: Joints and knobs activated on the slit-lamp

\subsubsection{Actuation of Slit-Lamp Arms}

The binocular-microscope arm and lamp arm were obvious candidates for the position controlled servos because of a limited rotation range under $180^{\circ}$ and the need to know the position at all times including when position limits have been reached. Also, these two arms have interconnected motions in that any rotation of the lower binocular-microscope arm also causes the lamp arm to rotate to the same degree as well. The position feedback allows for 
adjustments to be made so that when the binocular arm is rotated, the lamp arm can be counter-rotated to remain in the same position relative to the patent, if desired.

In order to achieve the goal of making the actuators easy to install and remove, adjustable worm-drive steel band clamps were used to hold the servo mounting brackets securely in place as shown in Figure 5-6. These were simple, strong, and readily available in all the sizes that were suited for this project. The vintage slit-lamp shape dictated that all the areas where these servos could be mounted were round and angled. The clamps were mounted just under each joint so that the binocular arm servo was mounted to the slitlamp base and the lamp arm servo was mounted at the base of the binocular arm. Some modification was made to the clamps to allow them to fit into the narrow spaces, but the goal was achieved.

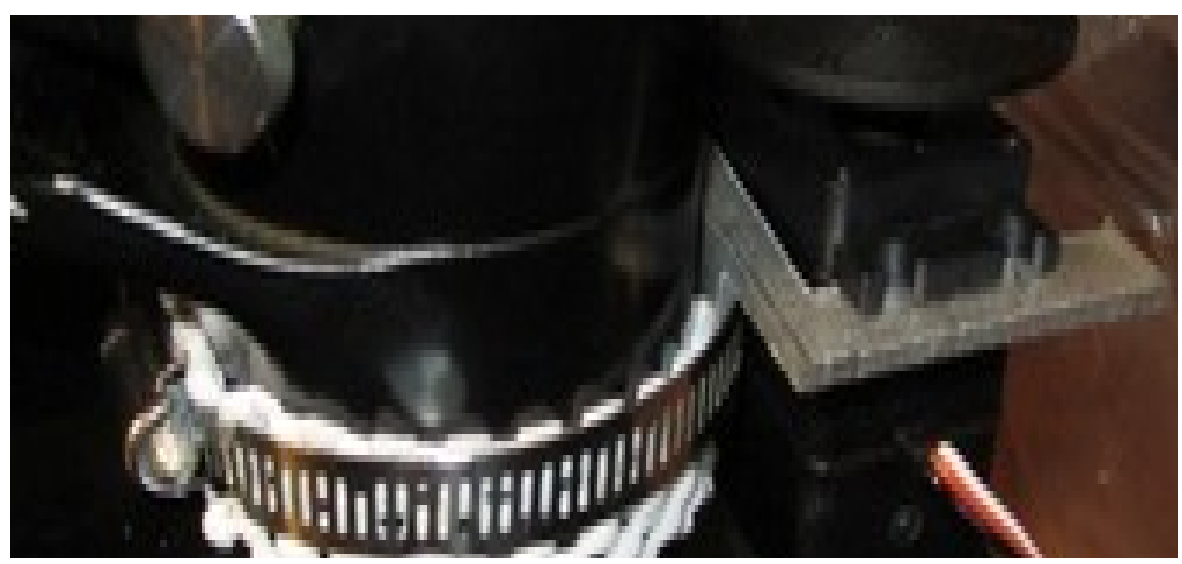

Figure 5-6: Clamp and aluminum bracket used to hold servos to slit-lamp 
The servos were attached by the clamps with custom made aluminum brackets. These had one surface shaped like a C-bracket that allowed for the servos to be secured via their four screw mounts and a second surface perpendicular to the first that was a tab that allowed for the bracket to be secured between the slit-lamp and the clamp. This can be seen in detail in Figure 5-6 above. Rubber material was placed between the slit-lamp, bracket tab, and clamp to ensure a snug and slip-free mount.

The servo outputs needed to be connected in a way to cause the arms to move. There were two possible options for this to occur. The servos could be fitted with gears that engaged with gear strips attached to the slit-lamp, or direct friction-drives could be used. With preferences to try to make the retrofit as simple as possible, be used as a universal kit, and allow for manual use, the friction-fit drive system was selected. It required fewer parts and nothing had to be permanent attached to the slit-lamp. This also allowed for easy and quick adjustments during the prototyping since relocating a servo would be less labor-intensive.

Rubber discs were attached to the disc servo horns. These were slightly over-sized to make the servo outputs contact the slit-lamp with increased friction between the servo output and the slit-lamp. The slit-lamp surface was also made to be rougher to aid in this endeavor by light sanding. The resulting implementation is shown in Figure 5-7 below. 


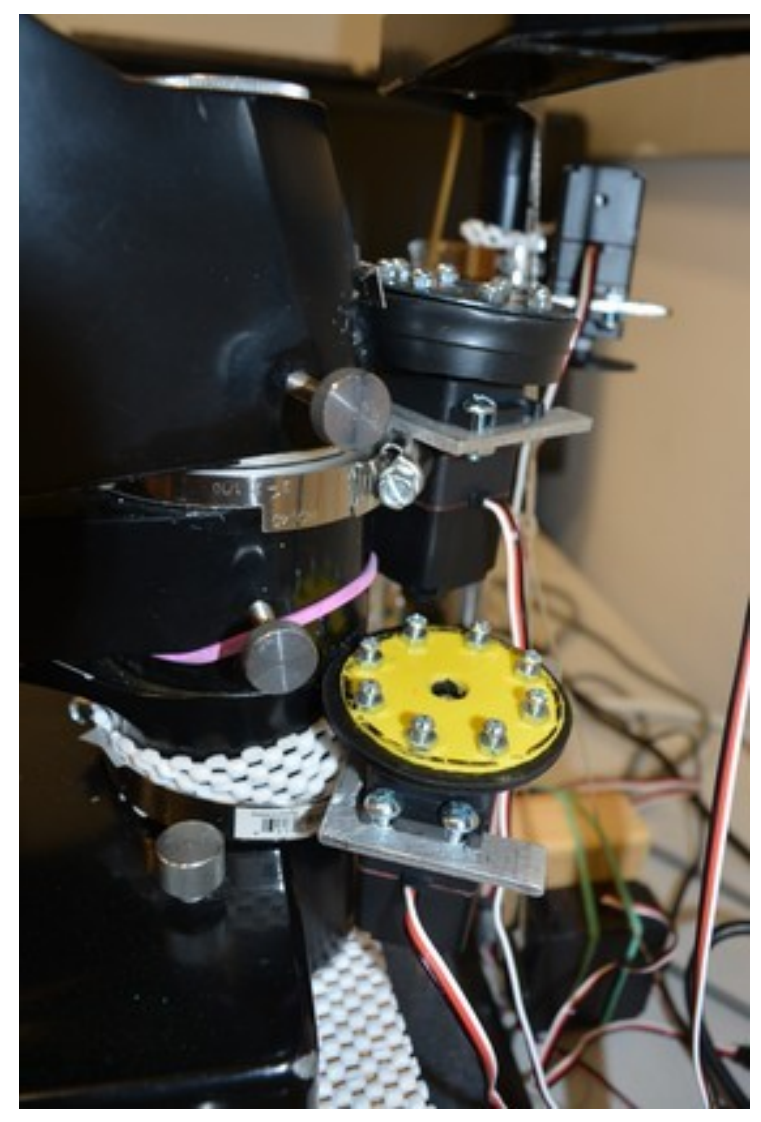

Figure 5-7: Actuation of the rotational arms of the slit-lamp

\subsubsection{Actuation of Slit-Lamp Knobs}

The control knobs for both the lamp light width and chin rest were actuated in a very similar manner as noted above. The light width also used the digital robotic servos, but the chin rest required the use of the continuous rotation servos due to multiple revolutions being necessary.

The light width servo was clamped securely onto the slit-lamp as in the previous cases and is shown in Figure 5-8. The knob then rotated as the rubberized circular servo horn rotated. 


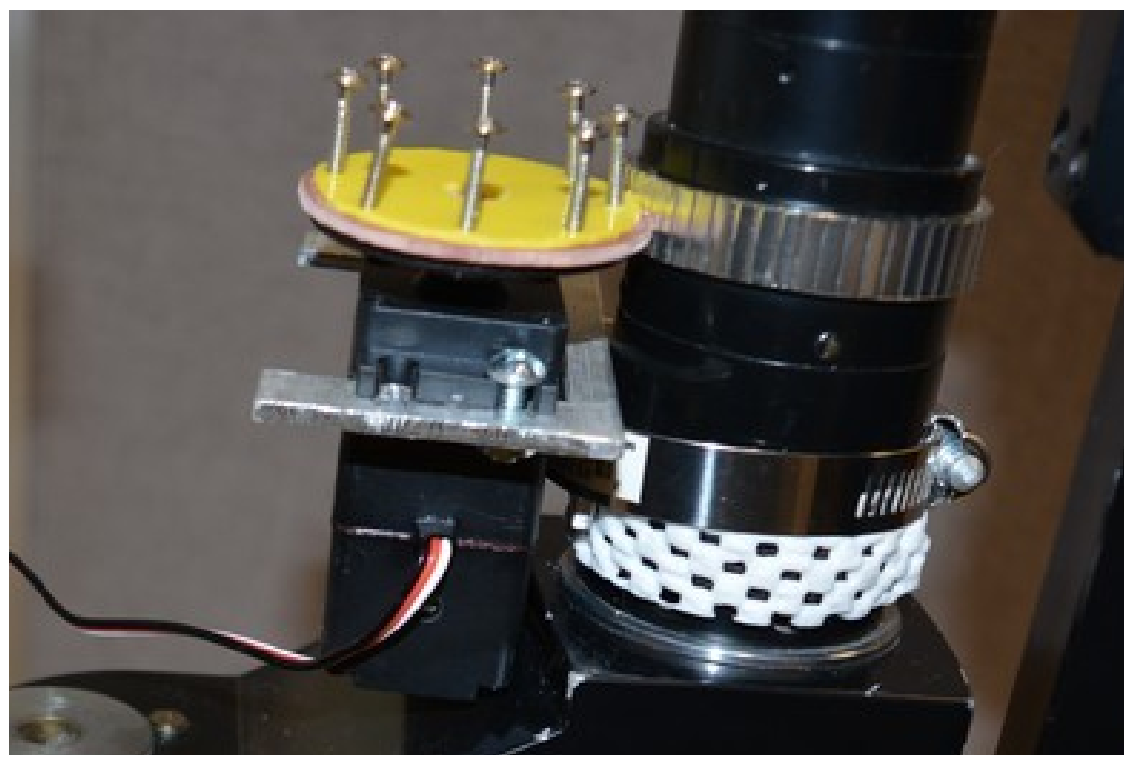

Figure 5-8: Actuation of the light width adjustment knob

The chin rest was actuated similarly to examples above, but with two differences. Due to the fact that the adjustment allows for more than three full rotations of the knob, a continuous servo was used. Next, the servo was mounted upside down so that the it was attached near the chin rest support bar as shown in Figure 5-9. This made it such that the relative position of the servo to the knob did not change during operation as the chin rest support moved up and down along the pole. 


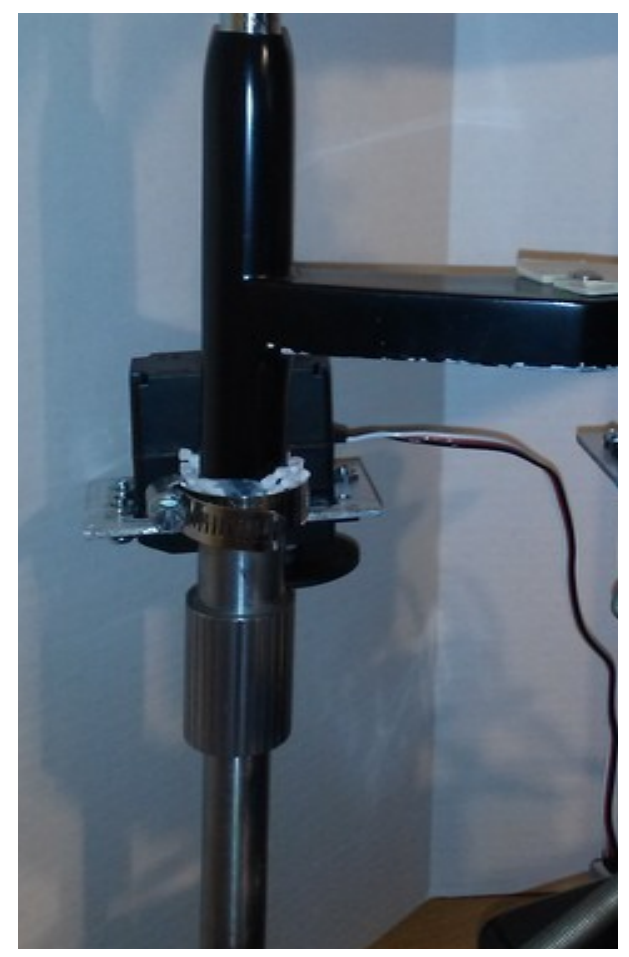

Figure 5-9: Actuation of the chin rest height adjustment

The slit-lamp height adjustment required a different drive mechanism solution. While similar to the chin rest in that the knob moved more than one full rotation, there was not a secure place to anchor the servo so that it remained in complete contact with the knob throughout the range of motion. This is because the knob moves upwards from the base of the slit-lamp as the height is raised. There was no secure place to attach the servo from above since thee was only a very small tab without sufficient area to securely mount the servo and the binocular arm above allowed for no clearance to build a secure bracket. To solve this problem, The servo bracket was mounted to the base of the slit-lamp. A thread spool was used on top of the servo horn to 
guide a belt and transfer the torque from the servo to the adjustment knob. The height of the spool was sufficient to allow the belt to freely travel upwards and downwards as necessary as the adjustment knob shifted in these directions so that the belt did not come off. The spool was later replaced with a 3D printed version for aesthetic reasons as shown in Figure 5-10 below.

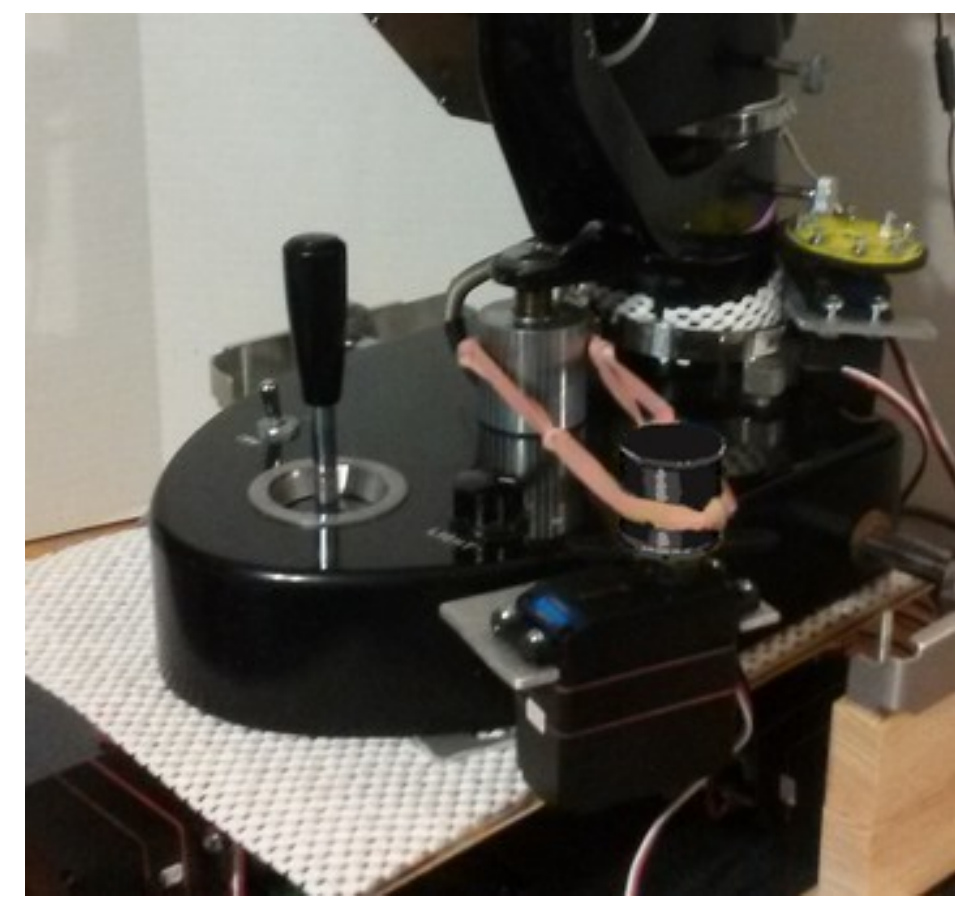

Figure 5-10: Actuation of the slit-lamp height adjustment

\subsubsection{Actuation of Slit-Lamp Base}

Actuating the movement of the base called for a completely different method of implementation. This motion requires the back-and-forth and left-toright motions of the entire slit-lamp within the range of motion allowed by the 
retaining tracks. The two main possibilities of actuating the base included using a wheeled or tracked system, or using a linear-actuated planar table mechanism. Based on previous work in Thailand [21], it seemed that perhaps a mobile platform underneath was the easier option.

It was decided to use Omni-wheels as this would allow movement back and forth in both directions in the plane. These wheels are not well suited for some applications such as moving on inclines or in rough terrain, but in this case they are used on a completely smooth and flat surface. This is an ideal application in many respects because they allow for motion in both linear directions. To move in one direction, the large wheels of one set mounted on an axle are activated while the other set, mounted perpendicular to the first, remain motionless with the small rollers acting as free-wheels. The underside of the base showing the implemented wheel configuration is shown in Figure 5-11. These wheels allow for an immediate direction change by changing the activation of one set of wheels to another. 


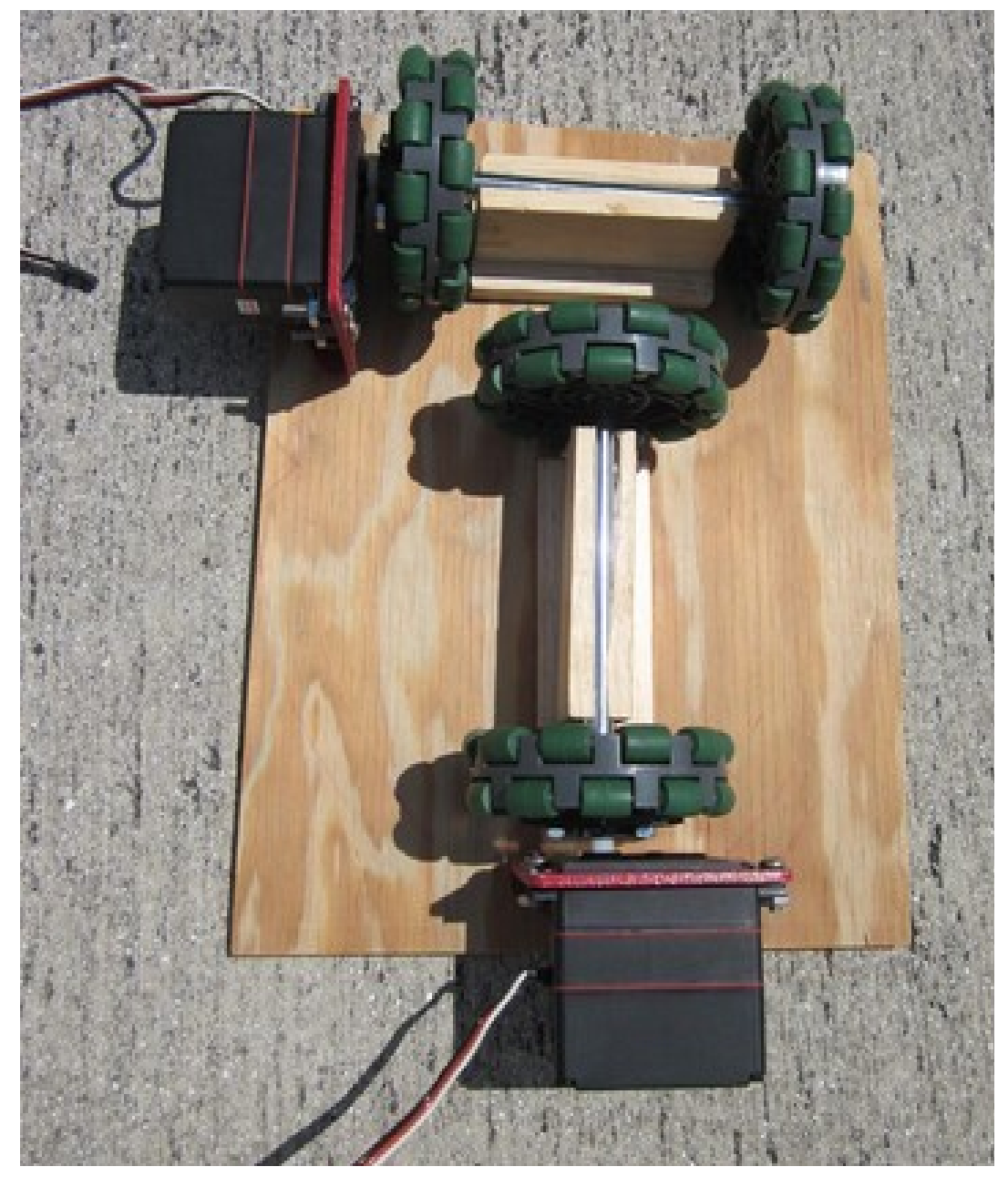

Figure 5-11: Underside of the slit-lamp base with Omni-wheels mounted

The original track that kept the slit-lamp on the table and within the bounds was retained to be used for the same purpose. The use of the Omni-wheels with this track made the motion of this system functionally similar to a linearactuated table, but with less complexity and precision needed in machining. This kept the cost down and is also easier to develop as a universal kit. Keeping with the goal of making the system modular, the base was made as a 
separate unit and the slit-lamp was then attached to the top. The mounting of the wheels was done such that the axles would be perpendicular as required for Omni-wheels, but that the slit-lamp would have its weight balanced on top of the wheels as shown above in Figure 5-11. A servo was mounted to rotate each set of wheels. The result with the slit-lamp mounted on top is shown in Figure 5-12 below.

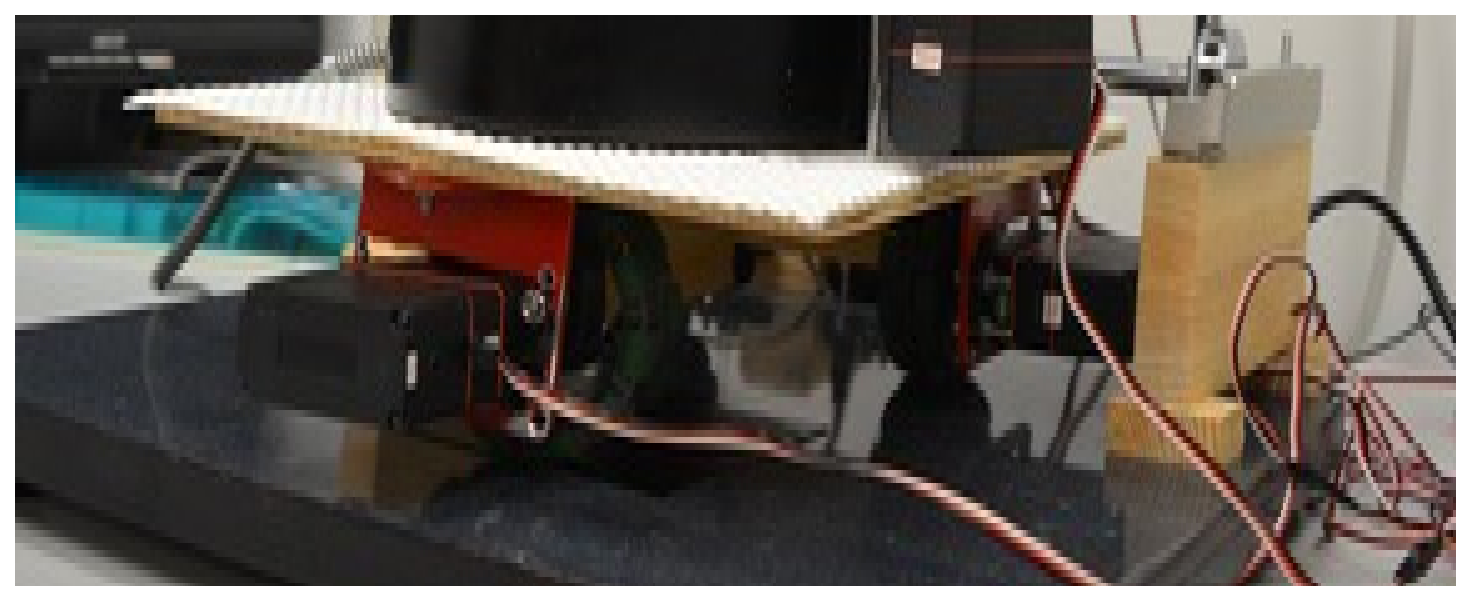

Figure 5-12: Actuation of the slit-lamp base with Omni-wheels

\subsection{Control Hardware}

Selection and implementation of the control hardware for this project went hand-in-hand with the actuation method. Again, the ease and speed of prototyping was a critical factor in selecting components. As noted previously, the Maker Movement increased the offerings of well-documented, costconscious and powerful options for this project. 


\subsubsection{Control Hardware Selection}

A full PC is used in conjunction with laser procedures as it is the controller for many ophthalmic laser systems, as shown in Figure 5-13. This PC may be modified and used in the control of the robot in a future iteration, but the use of a full PC for just the automation of the slit-lamp is not necessary. Since little to no autonomous function is given to the robot, the necessary computational abilities are limited. Furthermore, the system is intended for use over a longdistance communication line and cloud-based computing resources will likely be available. The most intensive processes are those involved with image processing and eye tracking and would require more intensive processor power for full real-time implementation. However, simple eye tracking has already been available on smart phones for years, so again the processing power needed is limited. Due to these facts, a single-board computer was deemed acceptable for this device. In addition to being small and cost-effective, they offer direct access to processor GPIO pins so that interacting with physical hardware is streamlined and avoids additional hardware to link the bus to the outside world. 


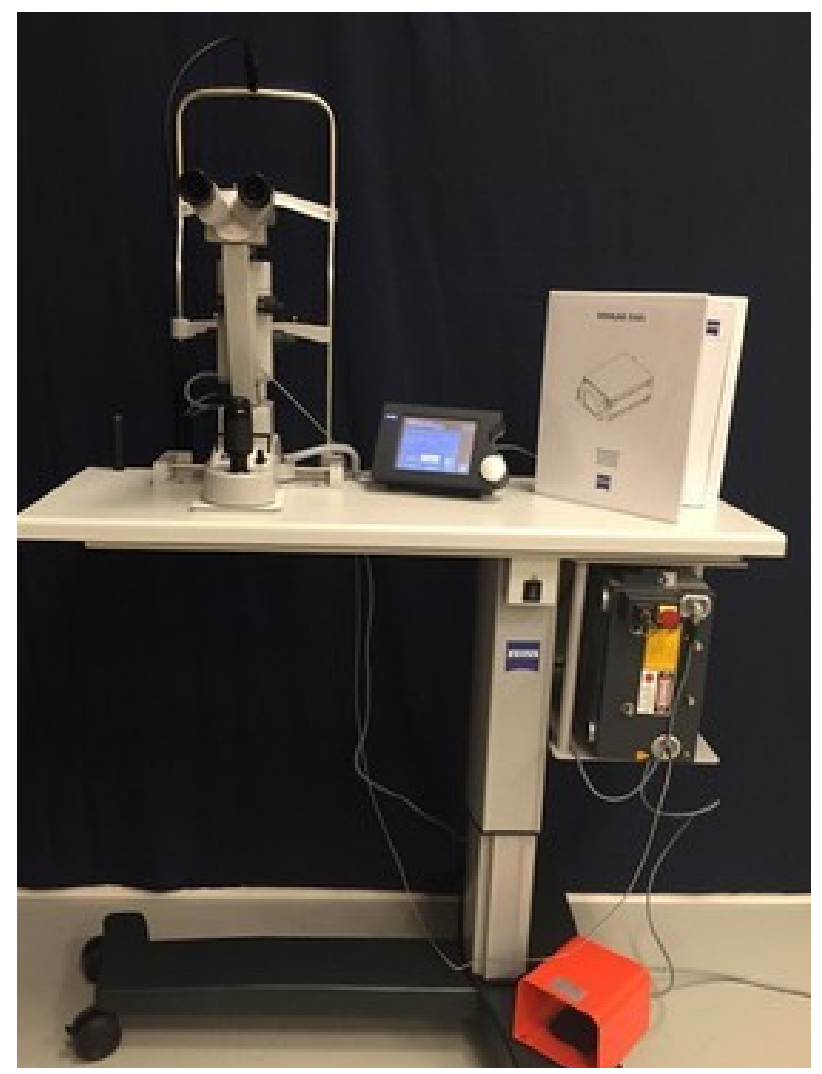

Figure 5-13: Full commercial laser and slit-lamp system (Zeiss)

Though several single-board PCs were considered, only two stood out as good options considering features, cost, and availability. These were the Raspberry $\mathrm{Pi}$ and the BeagleBone Black. These devices are very similar, though at the time that this project was implemented the Raspberry Pi offered slightly better graphics and the BeagleBone Black offered slightly better processing and memory. Though both aspects are important to this project, the overall control of the robot was the first and most important stage and so the BeagleBone Black Rev C, shown in Figure 5-14 was chosen. It was decided 
that handshaking with a Raspberry Pi could later be considered, if necessary, when implementing the image processing portion of the project in order to increase capabilities for eye tracking tasks.

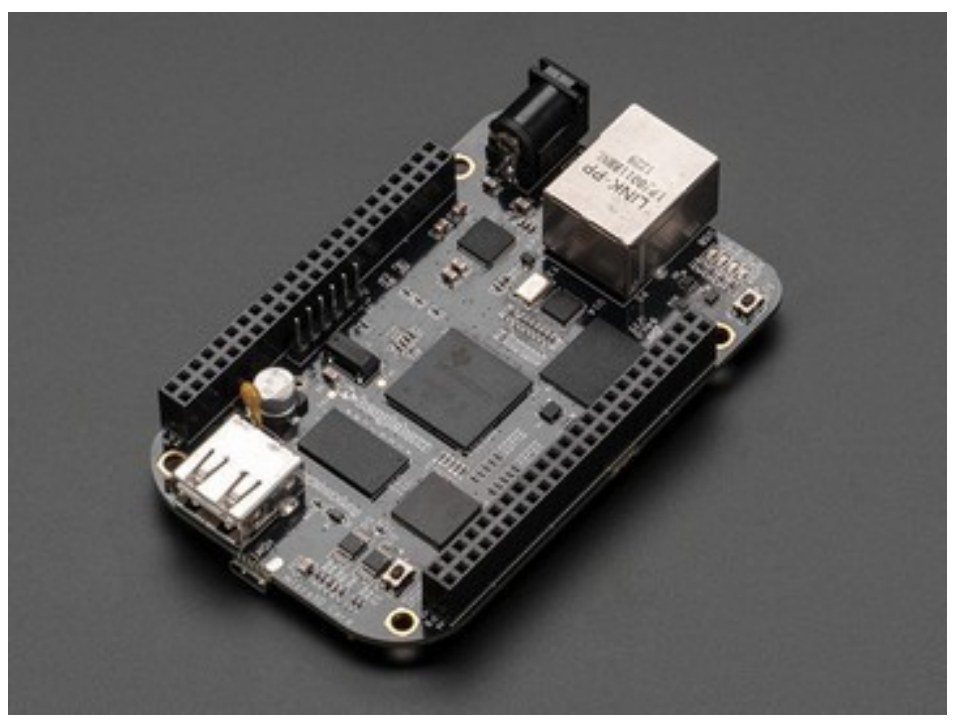

Figure 5-14: BeagleBone Black

While the BeagleBone Black offers direct access to GPIO, additional hardware was utilized to more efficiently direct servo motion. The Adafruit 16 Channel 12-bit PWM/Servo Driver 1411 shown in Figure 5-15 was selected to be the servo driver. With the ability to control up to 16 servos, it had sufficient capabilities to handle all of the actuators used in this project while only using two BeagleBone GPIO pins for i2c communication. 


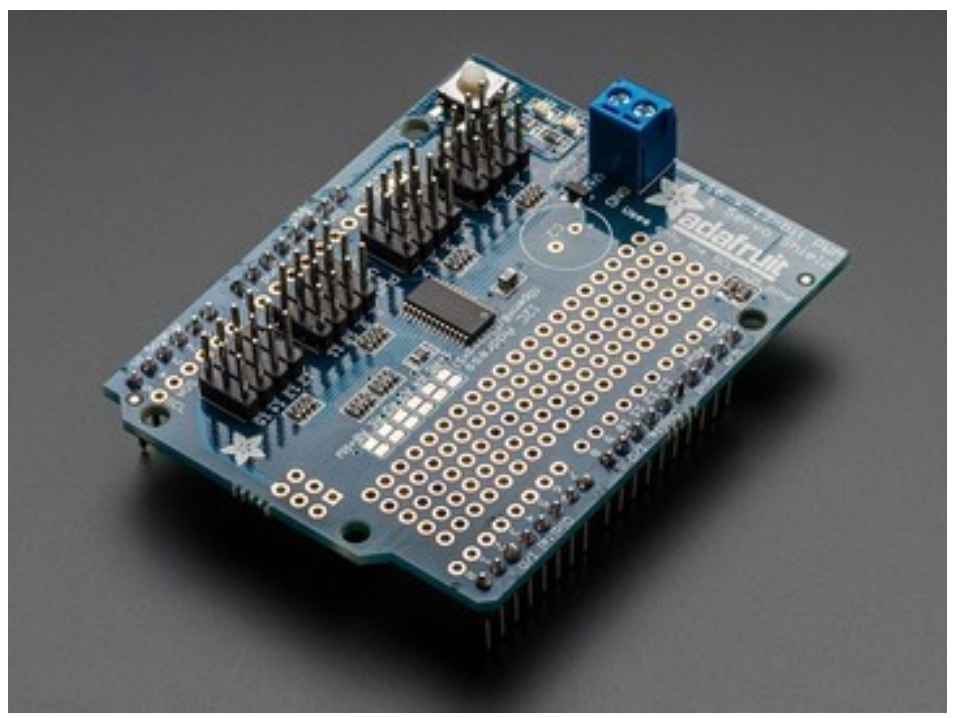

Figure 5-15: Adafruit 16 Channel 12-bit PWM/Servo Driver

\subsubsection{Control Hardware Implementation}

Implementation of the control hardware involved first wiring the separate components described in the previous section together. This was done by connecting the $\mathrm{i} 2 \mathrm{c}$ lines from the BeagleBone to the Adafruit PWM/Servo Driver as well as their power and grounds. A 5V, 20A power supply was connected to the servo driver to ensure sufficient power to the servos. The BeagleBone had its own plug-in wall transformer to provide power and offer some isolation if the servos drew a lot of current. All of the servos were then connected to ports on the servo driver with the output port numbers corresponding to those used in the software program. Finally, since the BeagleBone was usually connected to a PC for programming purposes, the PC was used to offer it connection to the Internet. In later uses, a WiFi dongle or direct Ethernet connection replaced 
this. A pictorial schematic is shown in Figure 5-16 while a photo of the physical implementation is shown in Figure 5-17 below.

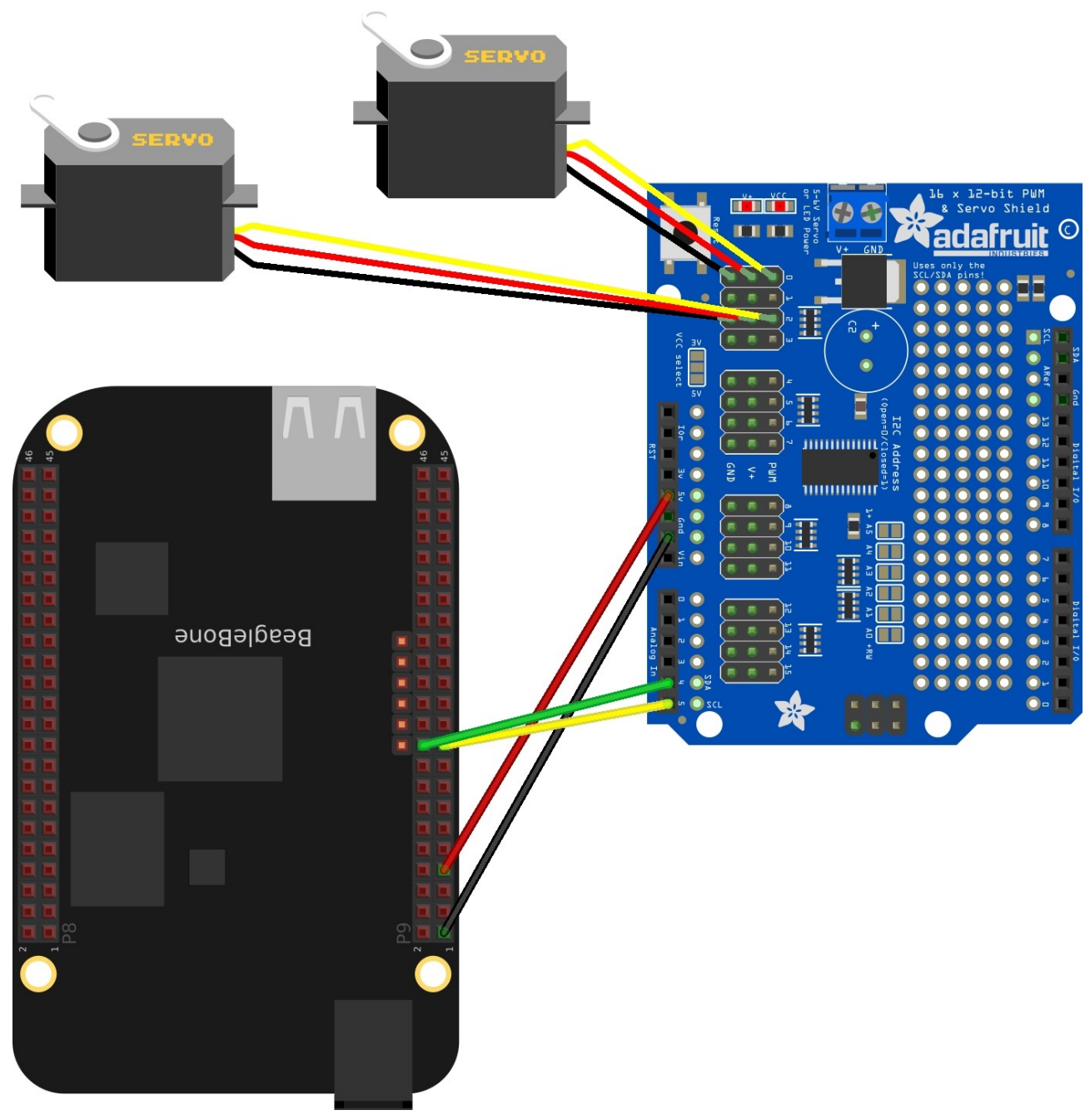

Figure 5-16: Pictorial schematic of circuit diagram for servo control system 


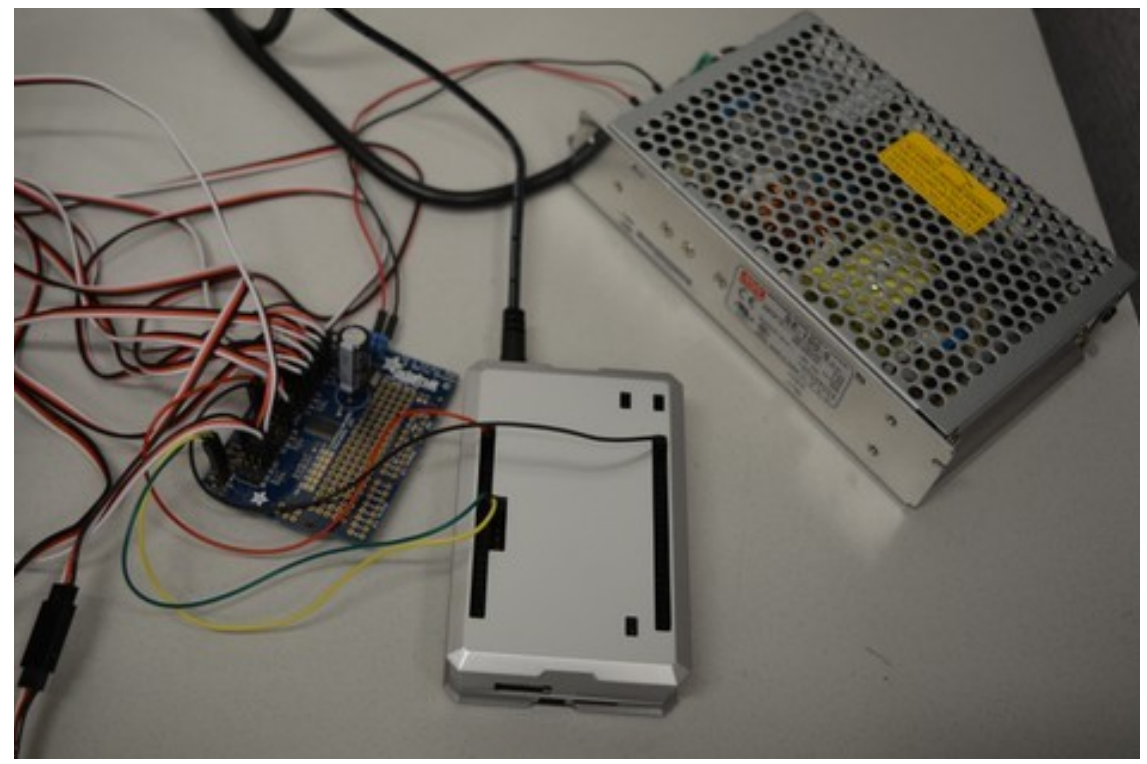

Figure 5-17: Implemented control hardware for the first prototype

\subsection{User Interface Implementation}

The patient-side interface is dictated mainly by the base medical hardware. Major components on the patient side are shown in Figure 5-18. These components as numbered are (1) robotic slit-lamp with control over moving parts and controls, (2) adjustable patient chin/head rest, (3) adjustable table, (4) on-site controller of laser and robot, (5) emergency stop button, (6) monocular camera, and (7) robotic lens holder. Not shown is the communication devices needed for remote access to the robot as these may be included in the controller or separately depending upon the method selected. 


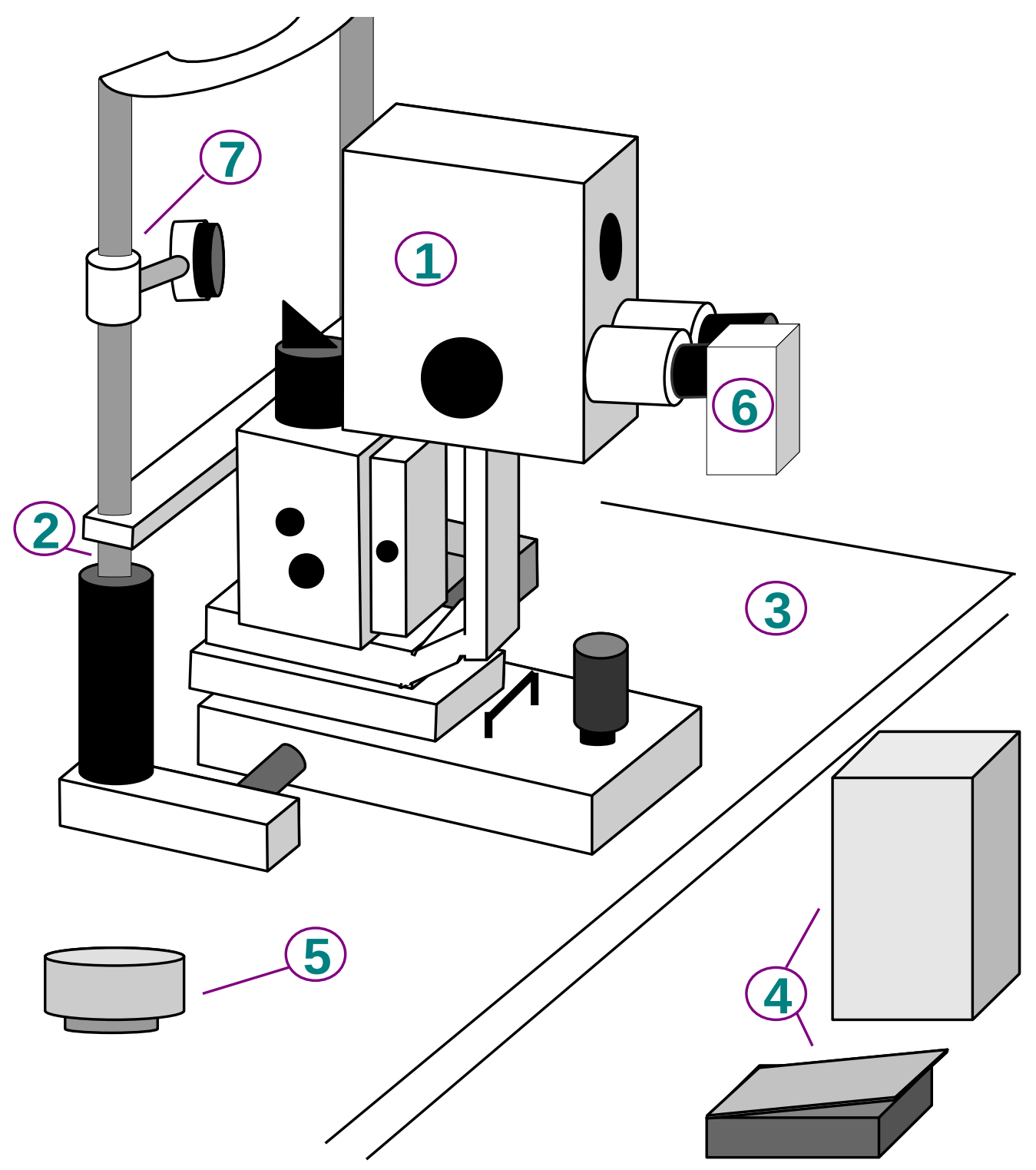

Figure 5-18: Patient-side interface

In regard to the remote controller part of the system, convenience of use for physicians from a variety of locations and devices is a primary concern when determining how to implement the user interface for the robot. The user 
interface must also be clear and easy to use to allow for safe and accurate control of the robot on the patient's of the system.

Current commercially available surgical robots have dedicated hardware control consoles [62]. The consoles tend to offer an integrated package including visual feedback from cameras, tactile control manipulators and buttons for robot control, and sometimes even haptic feedback. Ergonomics is an issue for some consoles [41] and is a concern for any interface used for long lengths of time. These large consoles are acceptable for current robot surgery uses as remote telesurgery is rarely performed. The physician travels to the location of the console and attends to the procedure in the operating room or in an adjacent room. The consoles are generally not moved notable distances once installed in the medical facility.

Different user interfaces were considered for this project and are proposed in the published patent for future options when the robot goes to market. These fit into three main categories and have their own advantages and limitations.

\subsubsection{Master-Slave Console}

A master-slave system is the first option. The control console consists of a physical device to manipulate the robot just as in current systems such as the da Vinci. This control console could vary in size and physical features, but the most obvious option is to use a full-scale slit-lamp shell as the controller. This 
follows the master-slave model for robot control where a passive (master) device that is a full-sized or scaled version of the active (slave) robot is manipulated to direct the motion of the slave robot. It is also possible to display the image output of the actual slit-lamp into the binocular portion of the master slit-lamp in order to make the experience three-dimensional and most like an in-person exam.

The slave side of the system can be seen in Figure 5-18 as already discussed. Major components of the master side of the system are shown in Figure 5-19 below. These components as numbered include (8) sensors for moving parts, (9) sensors for tactile control devices, (10) digital screens in false binoculars and/or use of a video monitor for patient-side monocular visual input, and (11) a method for activating the laser similar to that used in-person. Not shown is the communication method used to interface to the robotic side. This would vary depending upon the communication method chosen. On-site control hardware can easily be concealed within the faux slit-lamp to save space. 


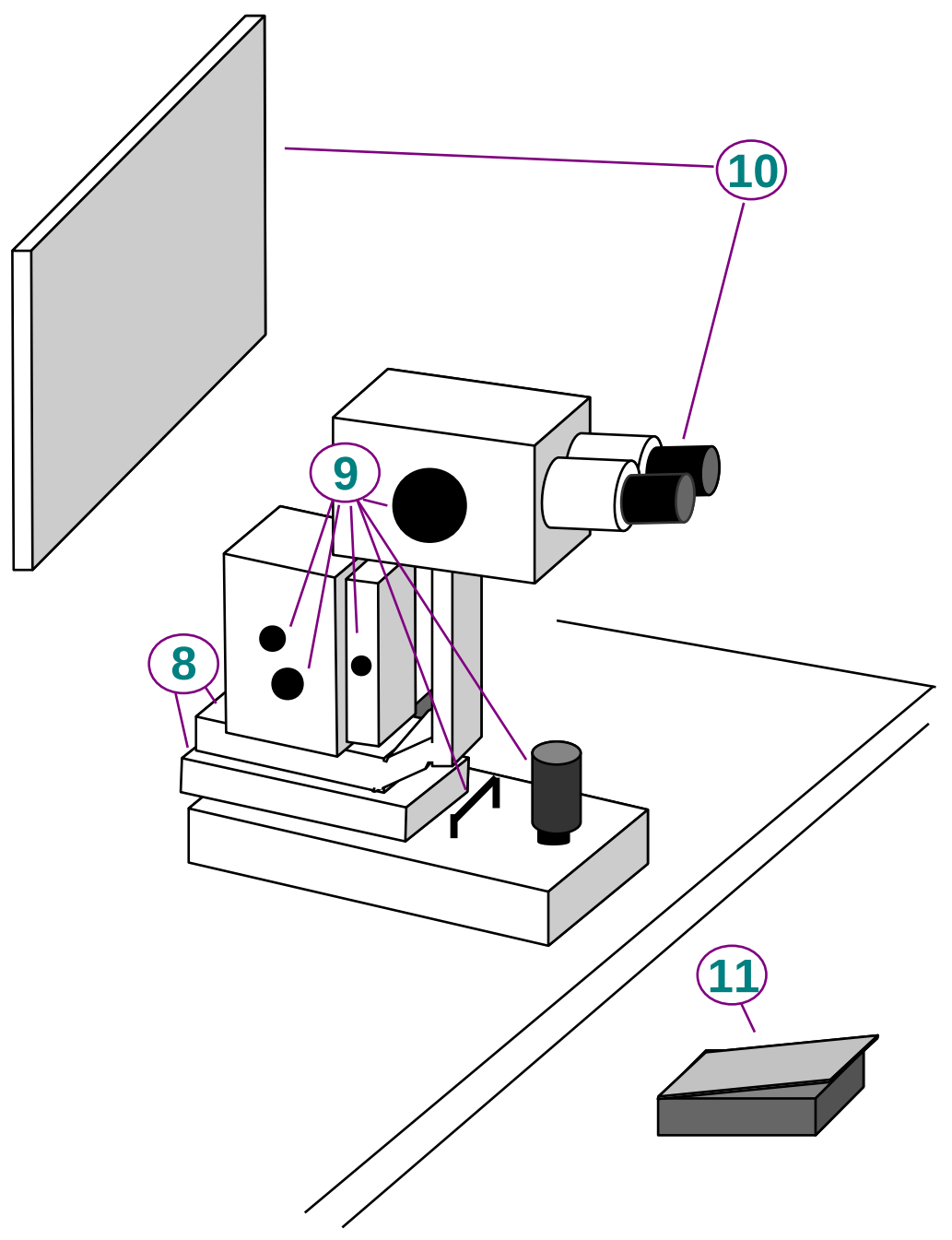

Figure 5-19: Master-Slave style control console

This would be the best control console for training as the physician will be able to apply the knowledge to both remote and in-person exams. It is also a great option for experienced ophthalmologists who would be more comfortable using equipment just as they have always done. This control console would be 
larger, heavier and thus less portable then the other options that will be presented. Though it could be made to be lighter than an actual slit-lamp, it will still be of such a size that it would likely remain in one location. It could be made semi-portable by use of a cart or installed in a vehicle. It would also be possible to have these installed in various locations such as clinics, hospitals and in physicians' homes so that they are likely to be near one of the devices, but it would not be something that could be kept nearby and ready to use at all times. In addition, the physical components and manufacturing of such a device likely makes this console option the most expensive of all of those presented. The increase in cost will increase the investment needed as well as limit the number of locations these consoles could be installed.

One big consideration in this type of console is that a master slit-lamp may not be very similar to the slave slit-lamp. While one option is to limit the slave options of each master console to those that are of a similar style, size and range-of-motion, this could pose problems as upgraded models of both the controller and robotic slit-lamps come to the market. As can be seen in many technology products, lack of compatibility causes increased costs with the need for further upgrades or case-by-case fixes. It would also mean that a control console used by an available physician may not be able to be used with a robot during an emergency. The unnecessary increases in cost and lack of prompt care are two factors that would defeat main objectives of the proposed technology. Another option is to develop built-in translation code to allow all 
master controllers to control all slave slit-lamps. Investigations must be made into how to implement this including kinematic detection, motion translation and unifying physician experience so that changing from one slave device to another will not cause any unexpected motions or mistakes.

\subsubsection{Portable Physical Console}

A more cost-effective and portable physical controller is an intermediary step in the line of possible controllers. In this case, the means of controlling the slit-lamp still involves physical components, but these components are reduced in size and weight. The package would either be made relatively small or would quickly fold to be a manageable form. The control console would typically consist of some type of joystick or similar primary motion control mechanism and tactile mechanisms for control of some features. One possible

example is shown in Figure 5-20 and has the following components: (12) A simple joystick mimics that which is used to make small adjustments to manual slit-lamps. Gross movements can be added by sensing larger input angles. Tactile buttons, knobs and switches can provide other movements and feature adjustments similar to the tactile experience of a manual slit-lamp. (13) Visual feedback can range from an external monitor, integrated LCD, or to video glasses. (14) Laser control can be separated for safety. 


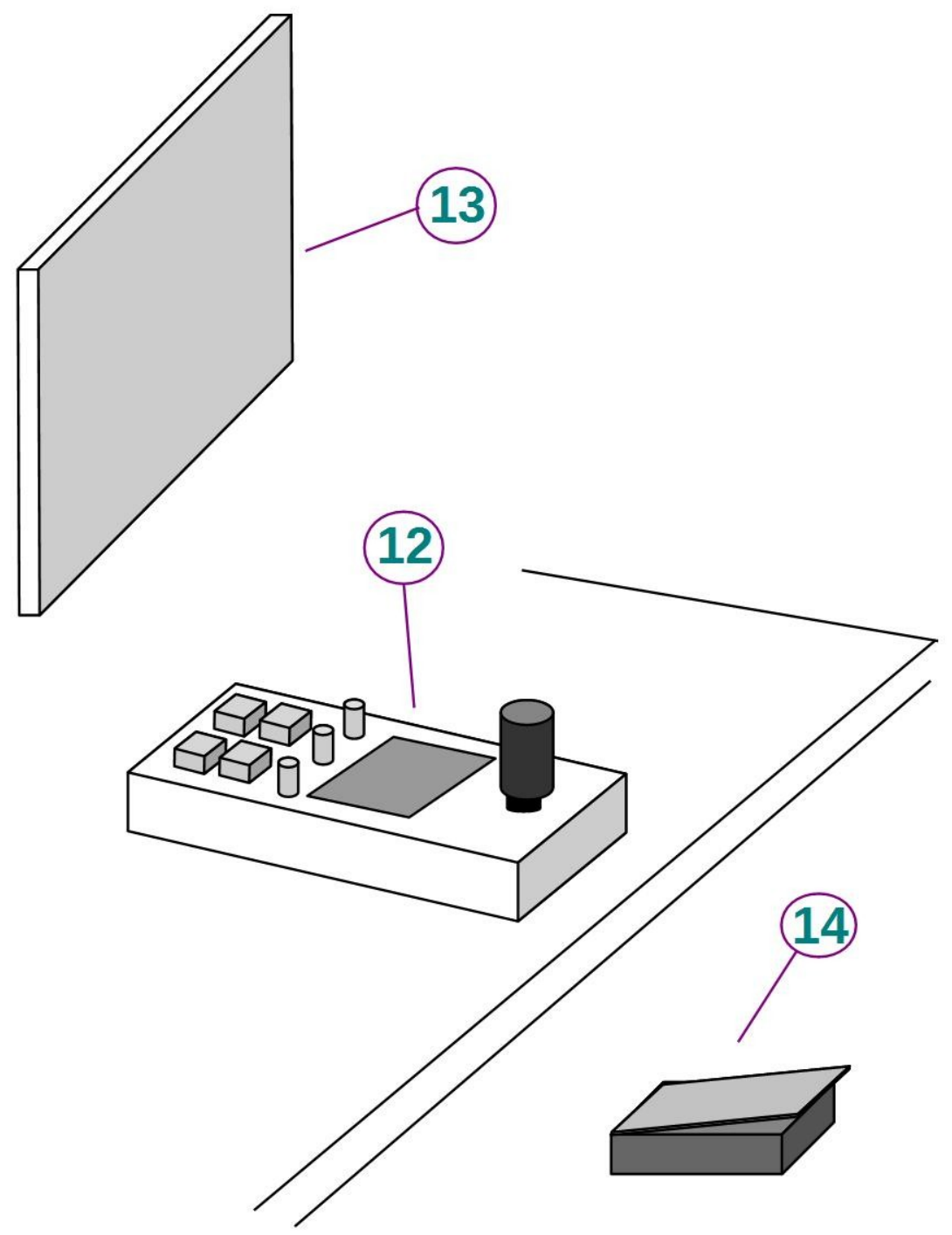

Figure 5-20: Portable physical control console

A portable control console allows a physician to still have the tactile experience of using a slit-lamp. It could be fitted with motors to also provide haptic feedback to confirm reaching end positions, indicate the robot hitting a physical object, or verify impending action. The device would be of a notable 
size, but could be stored within a small vehicle or possibly even a bag to be nearby at nearly all times. Unless controls are designed to be kinematically similar to a slit-lamp, the same generic control console could be used for any slave robotic slit-lamp without adjustment. Though there would be some manufacturing costs, all the components can be typical off-the-shelf tactile inputs so that the end product costs are kept to a minimum.

\subsubsection{Software Console}

The final option is to implement a software console. This would be a clickbased or touch-based console operated through a computer application or a website. An example of a possible interface is shown in Figure 5-21. This interface would be ideal on a large monitor or tablet in a landscape orientation. Modifications would allow it to be more easily viewed in portrait orientation or even on a smartphone for emergency situations. 


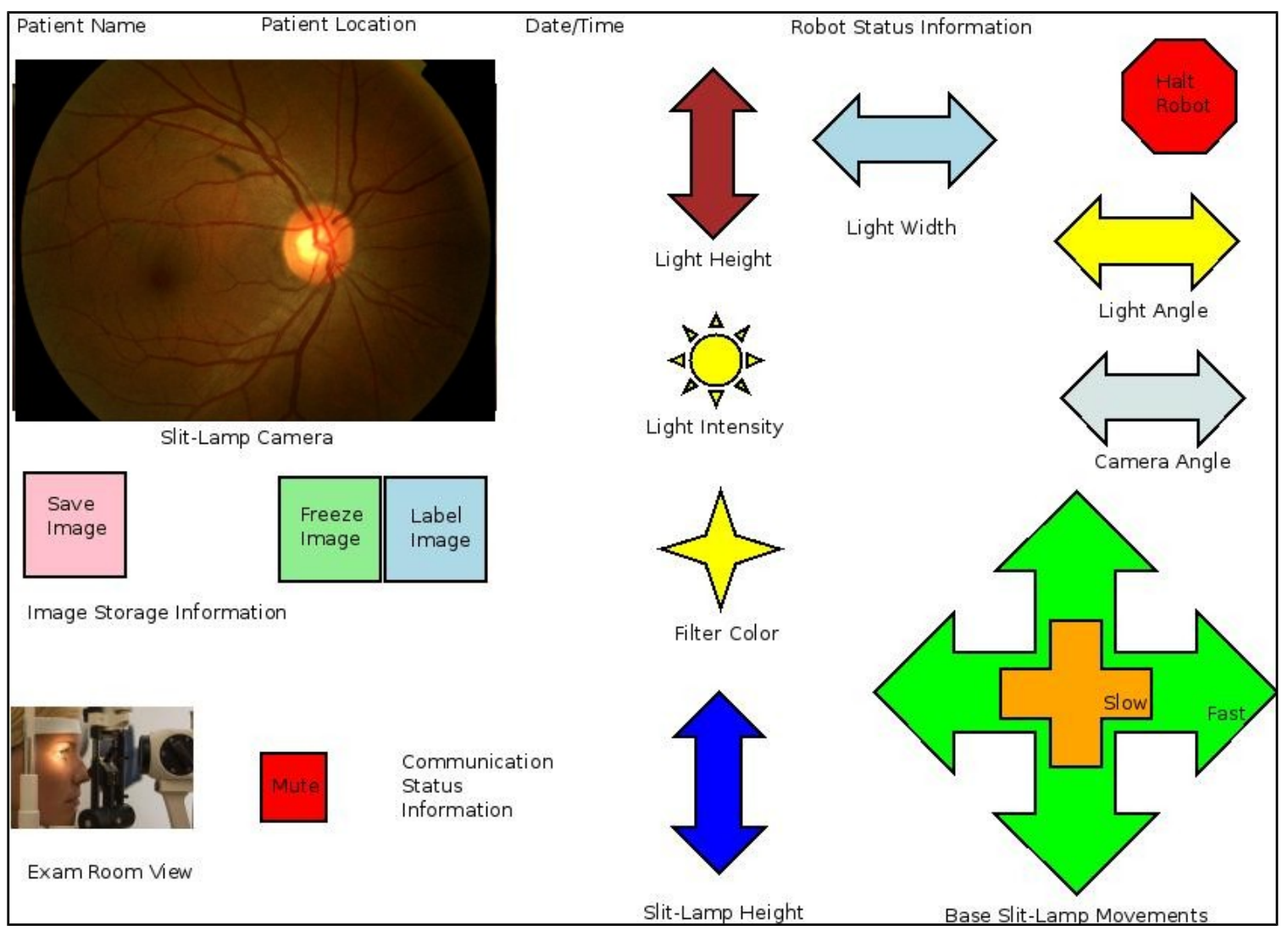

Figure 5-21: Software console

Clear advantages to this 'virtual console' include near-constant availability through use in nearly any computer. This includes desktop computers, laptops, tablets or even single-board PCs provided they can be connected to nearby monitor or screen. In an emergency, some functionality could even be provided through a smartphone with the main limitation being the screen size. In the case of devices that do not have a screen or large screen, the use of video glasses would allow for use with minimal equipment to carry. Using a 
nearby television or computer monitor is a possibility as well. All of these electronic devices are typically already available in hospitals, clinics and homes and so would not usually need to be purchased for the sole purpose of acting as a control console. They are easily carried and some are even are typically carried around. They already have built-in means for telecommunication and these can be easily used by the application or to connect to a control website. All of these allow for decreased cost in implementation of the robotic system.

Some problems are introduced, however. Current personal computing devices typically do not provide for any haptic feedback and will have no tactile sensations outside of some vibration. This means that the physician must be comfortable working completely through the software. Another problem is limiting control to qualified individuals. Once nearly any computer can access the robot, protocols must be put in place to ensure only expected and qualified individuals are able to access the robots at any time, especially while a patient is present. Finally, the use of equipment used for personal and casual use may have psychological impacts. Ophthalmologists may not approach every session in a professional mindset. It will be important to try to make the interface feel that they are in a clinical environment with the patient to aid in their mindset, much like pilots of military drones are required to wear battlefield uniforms for a similar reasons including mentality and professional custom [89]. 


\subsubsection{Prototype Console}

For this prototype, it was determined that the fastest and most flexible option was to use the later of the presented configurations. To allow for compatibility on a variety of devices without programming a version for every possible operating system, the web-based interface was selected. As this was to be a quickly developed proof-of-concept, issues such as security and professional mindsets were not a concern. These issues are expected to be addressed in later development and some may be alleviated by the use of dedicated applications and protocols rather than simple web pages.

\subsection{Lens Holder Implementation}

Other than being supported by the chin and head rest bars, the patient will only come into regular physical contact with one other component of the robotic platform. This will be lenses and the manipulator that holds them in place.

Lenses of varying properties are used to expand or enhance the visual access of the interior of the eye. They can magnify aspects or allow view of peripheral areas that are not in direct line-of-sight without aid. The lenses are of varying size and shape.

For the current stage of this project, it was expected that the on-site medical assistant places and secures the desired lens at the end of the manipulator and applies the lubrication needed between the lens and eye. This assistant also 
places the manipulator in position on the eye so that gross adjustment via robotic control is not required. In the future, a process similar to automated tooling changes can be designed and implemented to perform this task.

The lens must stay in consistent contact with the eye. If the eye and lens separate, then the view is altered or disrupted. At the same time, the lens must not be allowed to apply too much pressure on the eye. This could cause an altered view and in more extreme cases could cause damage to the eye. Maintaining this balance is a challenge. The patient can be expected to fidget and move slightly, even when told to remain perfectly still and possibly be aided in doing so by the on-site medical assistant. The eye is usually able to move around freely and all people have a certain amount of tremor and will fidget during long periods of time. Normally, a physician controls this pressure and makes adjustments based on what they see and feel. Ideally, the lens holder must have mechanisms to perform these tasks in addition to simply holding the lens and making simple adjustments to lens position.

Once the lens is in place in the lens holder and on the eye, the ophthalmologist will then be able to take over control and make more minute motion adjustments. This requires a small and accurate sub-mechanism that is a robot itself. As with any robotic manipulator design, multiple options are available and were considered. 


\subsubsection{Lens Holder Design Options}

The most traditional design would be that of a serial manipulator directly driven by actuators. This resembles robotic arms that are commonly used in manufacturing facilities. It is composed of a chain of rigid links connected by actuators from the base to the end-effector, in this case the lens bezel. An example is provided in Figure 5-22. It can easily be any number of DOF by simply adding or removing a link. Motion at any of the links can be rotational or linear.

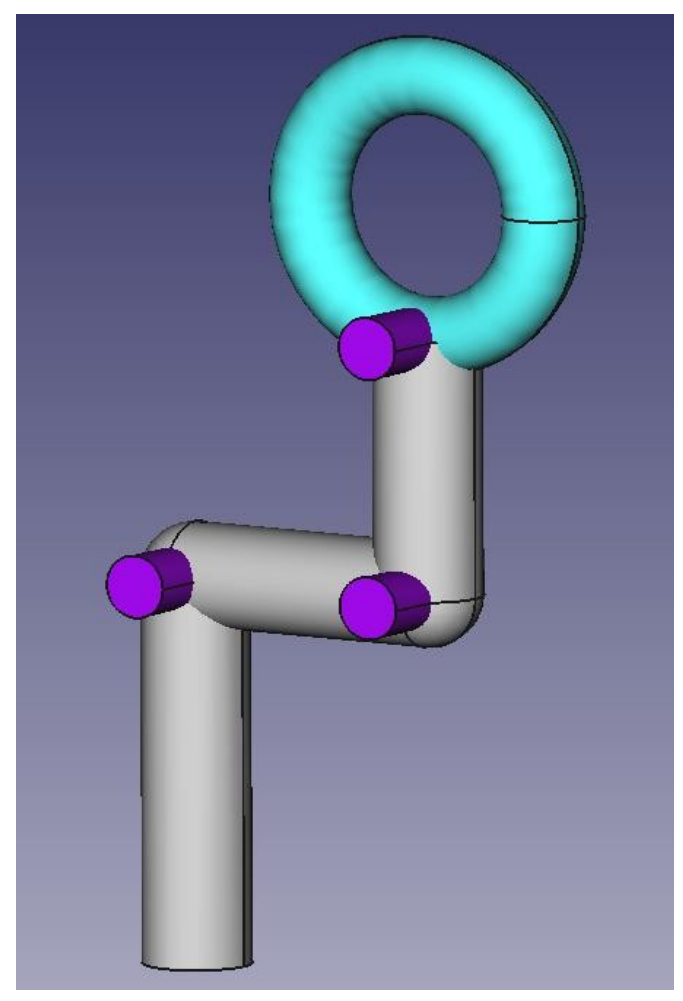

Figure 5-22: Serial lens-holding robotic arm general design 
A variation of the above design is to indirectly drive each link. This has the advantage of allowing motor placement to be more flexible. In this case, it affords the ability to locate the motors at the base of the lens holding arm in order to decrease the weight and size of mechanism near the patient's face. This increases safety in that less force is needed to move the lens and that there is less material and mass near the face. Different mechanisms can be used to convey the actuator movement to the links. These include cables (also referred to as tendons or strings) and belts. Due to elasticity in the cables or belts, these systems naturally tend to include notably more compliance than traditional directly-driven links. Though this can cause position errors, in this application it allows for a certain amount of passive tracking to keep the lens on the patient's eye during normal fidgeting without necessitating constant fastmoving adjustments in the lens holder manipulator arm. An example of this variant is is presented in Figure 5-23.

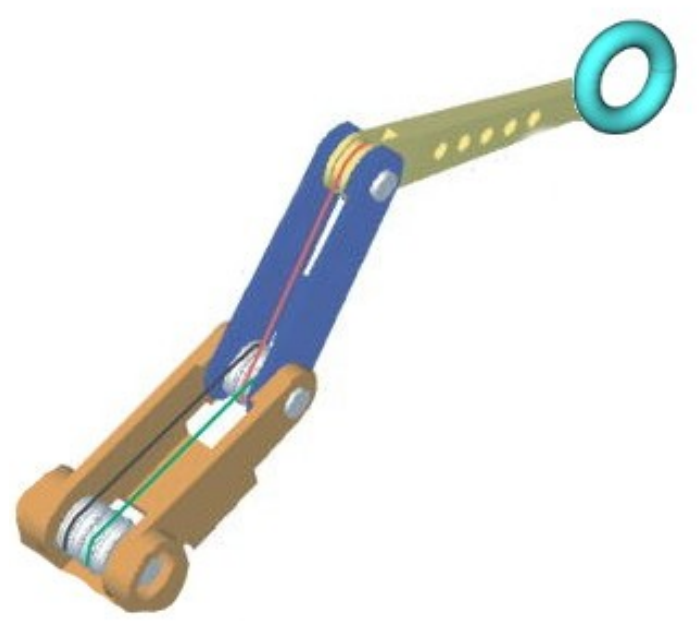

Figure 5-23: Compliant serial lens-holding arm. Image adapted from [121] 
The final main type of mechanism considered for the lens holder was a parallel manipulator. This type of manipulator generally is extremely rigid and accurate, though more complex and heavy. This would have the drawback of having more material in front of the face and also could lead to a lessresponsive system to larger motions. However, newer designs and material uses allow for it to be compliant or fast, however, so that it is still a feasible consideration for this application. Semi-flexible links or cable-driven motions both introduce some tolerance for the mechanism to passively adapt to small quick movements as well as to be built with less weight and material to be obtrusive to the patient's face. Some examples of these options are shown in Figure 5-24.

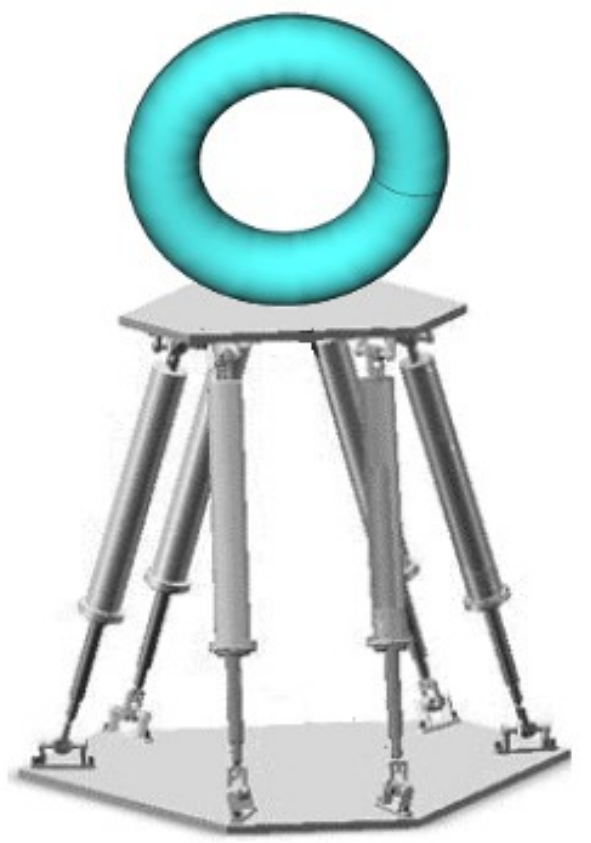

Figure 5-24: Parallel lens-holding designs. Image adapted from [24] 
Any of these options provide feasible options for holding the lens. They all allow the physician to remotely control the position of the lens in varying degrees. While the serial arms allow for larger motions, the parallel options also provide sufficient motions for most cases. The possibility of selecting materials and configurations to allow for passive compliance to maintain consistent contact with the eye are also present. This compliance could be adjusted by changing the material or cable tensions used in each design. Reliability, cost and comfort become the main deciding factors after technical aspects have been achieved.

\subsubsection{Implementation}

Simple analytical evaluations of the various lens holding arm mechanisms are not sufficient for determining the best option for use in the actual system. It is hard to account for human preference, both for the patient and doctor, in a simulated model. Also, actual properties of the physical unit after manufacturing change the expected performance somewhat. While minute, small changes on something as sensitive as the eye can have large impacts.

The best of the above options may not be fully known until after more extensive testing. Human and animal testing is a future part of this work, so an optimal design will not be definitively named here. Still, options were considered and tested to come up with some initial opinions. It was decided to try to start with those that would be the simplest first. This would require easy 
manufacture and passive compliance so that the system would not have to be as responsive in making small movements to maintain proper pressure on the eye. These designs would probably be the least expensive as costs would be saved in manufacturing, materials, actuation and control. In this regard, two different lens holders were implemented on the first prototype. The first used a serial arm with cable-driven links. The second was a parallel cable-driven mechanism. Both had advantages as well as issues to resolve.

The first lens holder is shown in Figure 5-25 below. This lens holder was a 2-DOF hollow serial arm driven by cables attached to servo motors. Springs were used to stiffen the joints, but allow for bending. Small internal pulleys were used to direct the thin wire cables so that the joints would move as desired. In the spirit of trying the most simple solutions first, the mechanism to actually secure the lenses to the arm was simply a small support with adjustable Velcro tape to wrap around. It was initially able to perform the tasks required and was installed for several months of initial testing. However, over time the springs tended to weaken and the pulleys were too delicate to remain in place with the forces that were sometimes applied to obtain positions needed. Overall, this is a very feasible design, but some adjustments to make it robust and more complicated manufacturing is needed. 


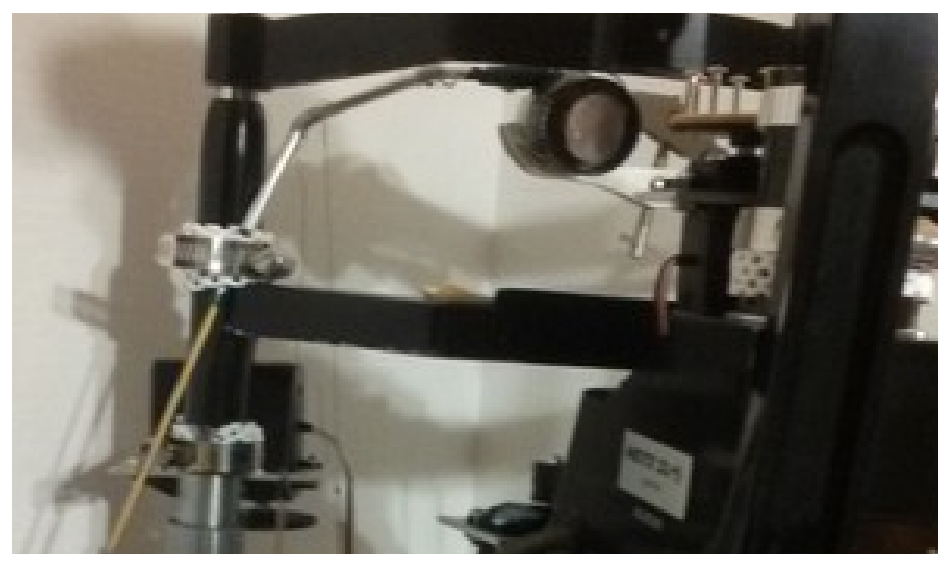

Figure 5-25: Initial lens holder

A better view of the actuation of the lens holder can be seen from the rear of the slit-lamp where the patient would approach as in Figure 5-26.

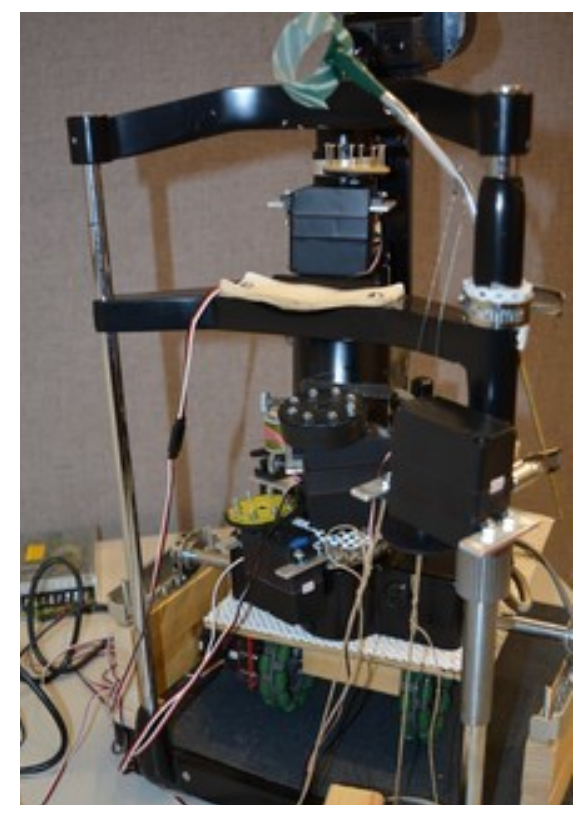

Figure 5-26: Initial lens holder actuation (from the patient's side of the slit-lamp) 
When replacing the first lens holder, it was decided to try an entirely different mechanism. While the first could have been made to work with further adjustments, it was desired just to see how a more conventional and simple mechanism would work on the actual system. This second iteration used a pan-tilt mechanism. It is shown below in Figure 5-27. The mechanism implemented was a commercially-available kit intended for small video cameras. The lens was held on by a round bezel mounted to the platform where a video camera would normally be mounted. Set screws held the lens in place and allowed for adjustment to accommodate various sizes of lenses. It proved to be much more robust against the forces acting upon it by the eye and held steady against movements of the slit-lamp. After testing, it was determined that a large amount of compliance was not needed for the lens holding component.

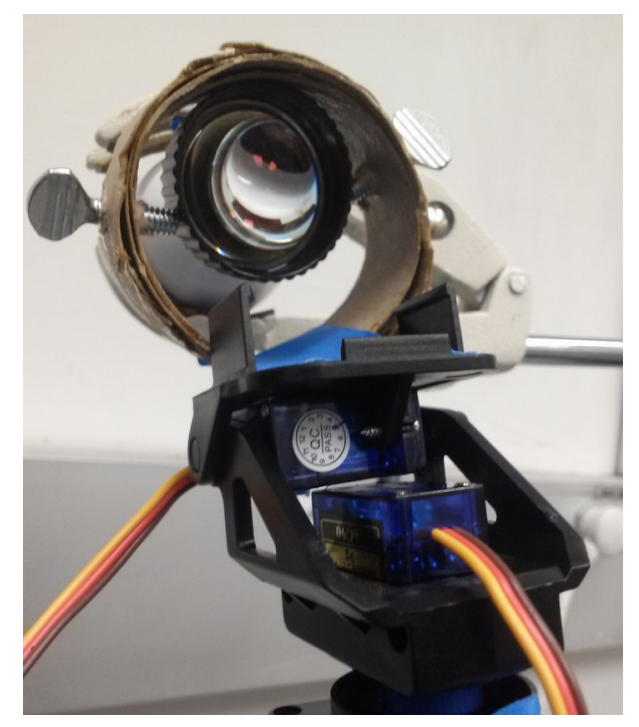

Figure 5-27: Pan-tilt mechanism for lens holder 


\subsection{Implemented First Prototype}

The first prototype, OphthBot 1 as completely implemented can be seen in the Figure 5-28.

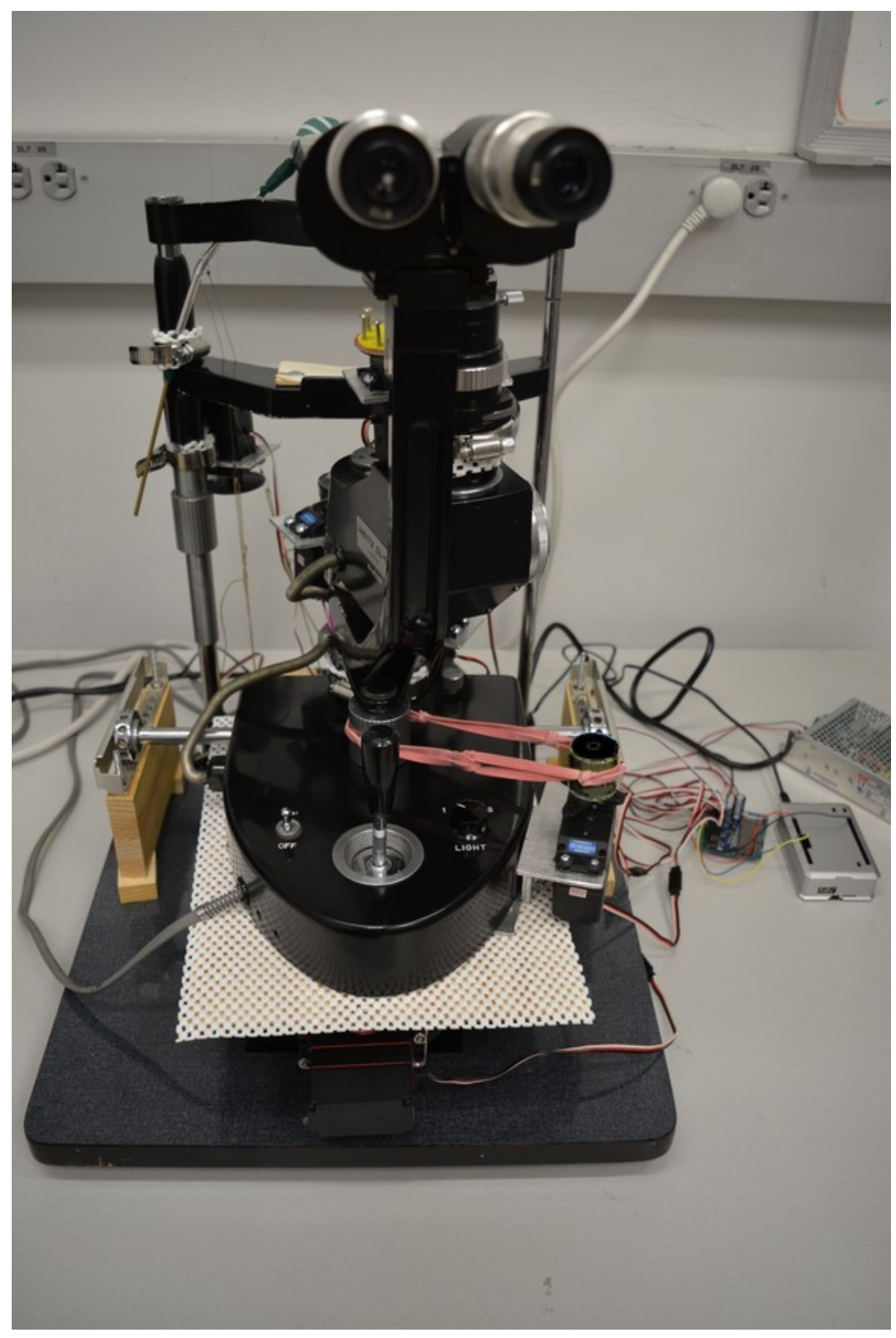

Figure 5-28: First robotic slit-lamp prototype - OphthBot 1 
More discussion about the operation and results from this phase of the projects are discussed in Chapter 8.

\subsection{Second Prototype Design and Construction}

The original intent of the project was to use an entirely different slit-lamp for the second prototype. It was planned to use a modern model with a laser installed. However, there were many improvements that could be made to the original prototype. Using the same slit-lamp on the second prototype allowed for the investigation and comparison of alternative design options that were not selected the first time.

Some changes in the goals were made for the second prototype, OphthBot 2. In this instance, making a universal retrofit kit was not a goal. Instead, the focus was making the actuation and control as accurate and robust as possible. This means that the parts used were made to custom fit the slit-lamp and would have to be uniquely designed and manufactured for any future model of slit-lamp.

\subsubsection{Automation of the Base Slit-Lamp}

The second prototype automating the slit-lamp was completed with the aid of a team of undergraduate students. Details were published in other works including [64], but a very brief overview is presented here. 
The automation of the base slit-lamp used alternative methods not selected for the first "kit-style" prototype. Most notably, in this implementation, an X-Y table, shown in Figure 5-29, driven by stepper motors and linear screws was selected for the base motions. In addition, all rotational joints were fitted with custom-designed gears that were 3D printed and permanently affixed to the slit-lamp as can be seen in Figure 5-30. An image of the slit-lamp portion of Ophthbot 2 is shown in Figure 5-31 below.

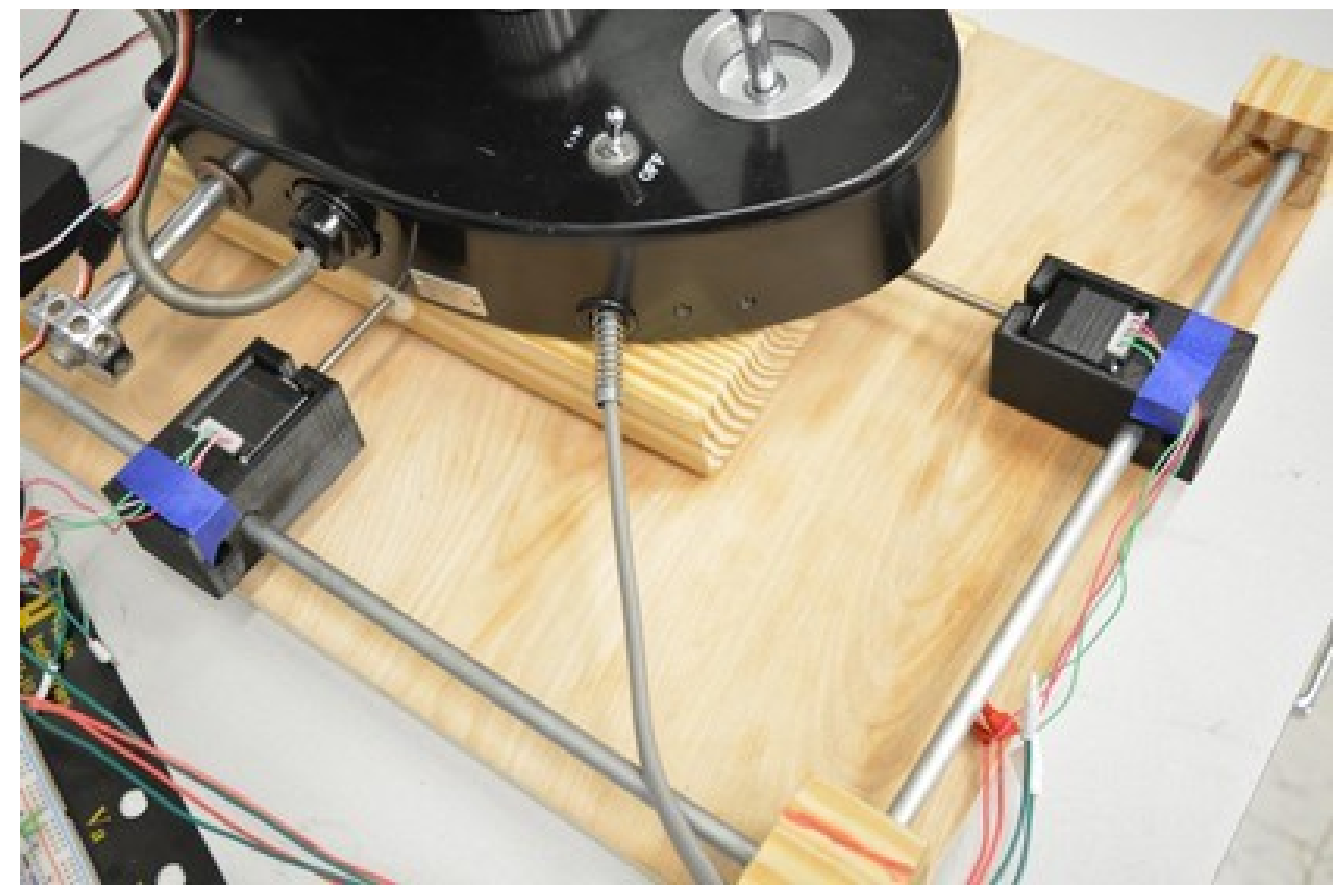

Figure 5-29: Stepper-driven X-Y table for base movements 


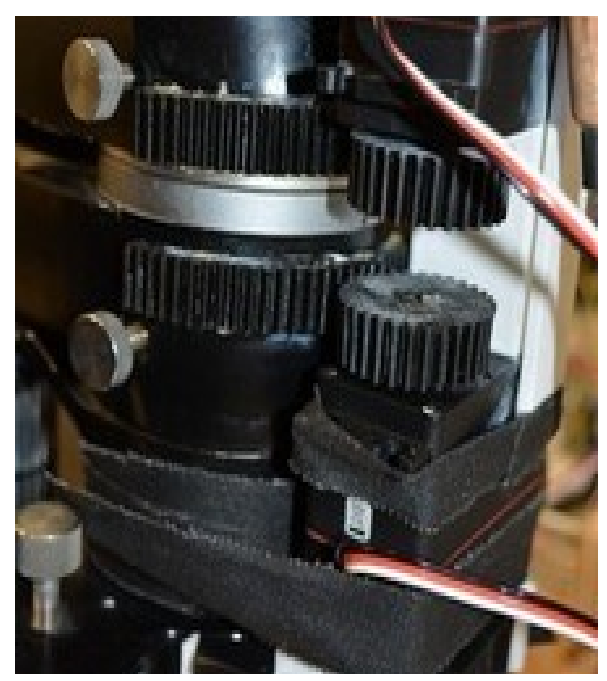

Figure 5-30: Geared servo interface to binocular and lamp arms

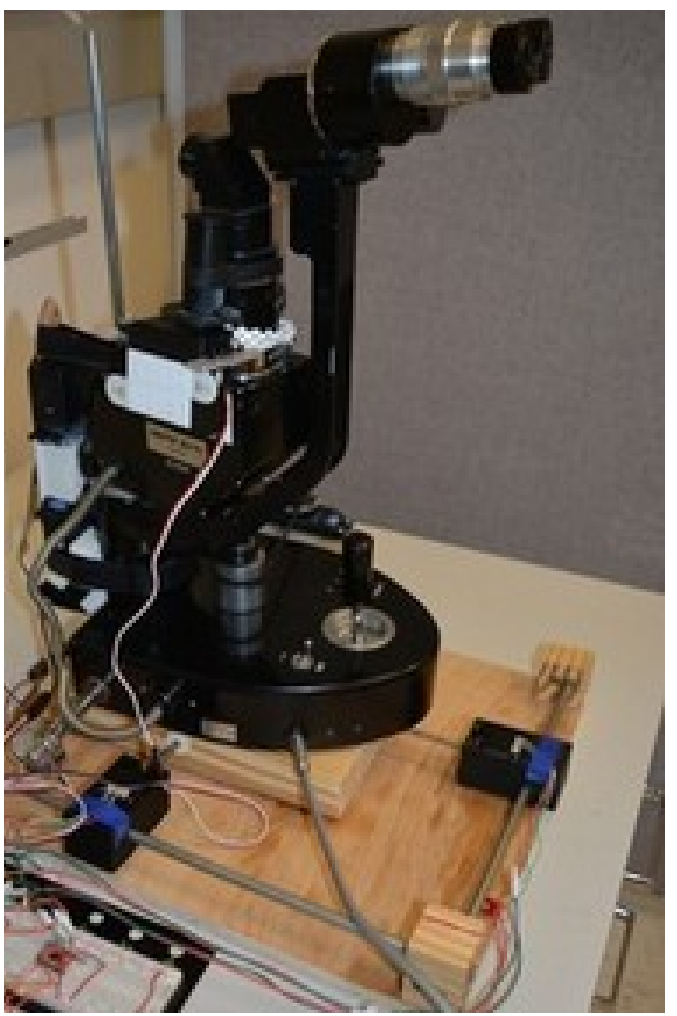

Figure 5-31: OphthBot 2 automated slit-lamp 


\subsubsection{Lens Holder Implementation}

The lens holder implementation also followed one of the alternative designs not used in the initial prototype. It was completed with the participation of a second team of undergraduate students with published details in other works including [52].

One of the more ambitious alternatives developed in the brainstorming processes, but not noted above, was chosen for this design. This design is shown in Figure 5-32 below.

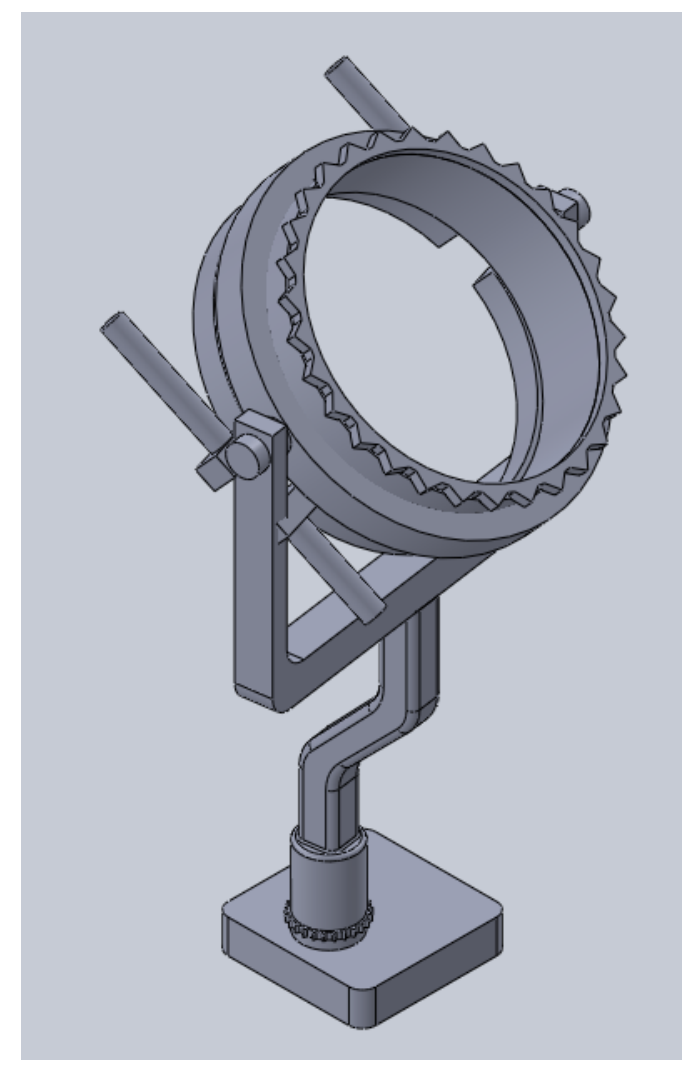

Figure 5-32: Design for OphthBot2 lens holder 
This design was pursued as it seemed to be the most promising after experiences with the shortcomings of previous iterations. The only additional feature omitted was a keyless twist-lock lens clamp on the lens holder bezel to allow for quick and easy lens replacement and fit adjustment. It would be similar to a keyless twist-lock chuck as is used in drills to hold various sized bits. However, set screws were a suitable alternative that offer better cost and weight specifications and these are implemented in prototype as shown in Figure 5-33 and Figure 5-34.

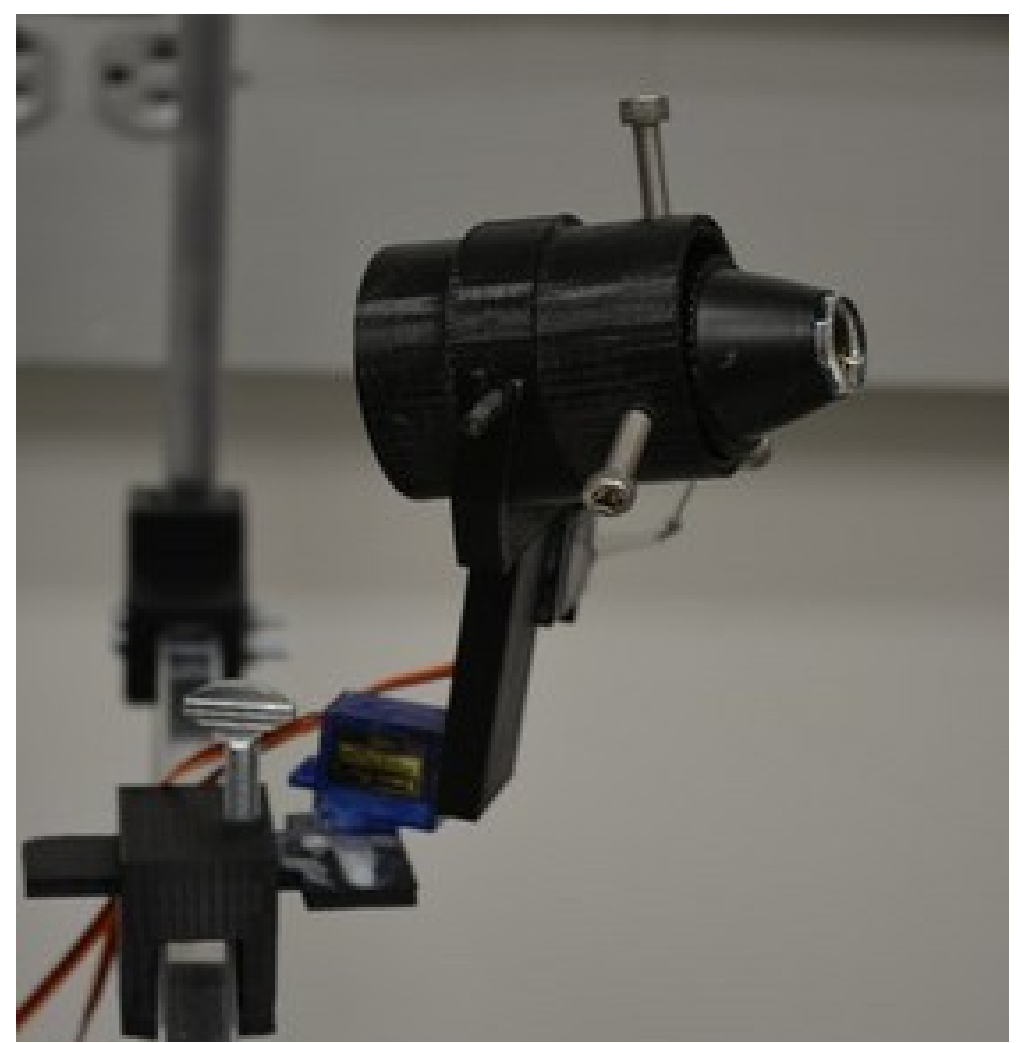

Figure 5-33: Close up of the implemented OphthBot 2 lens holder 


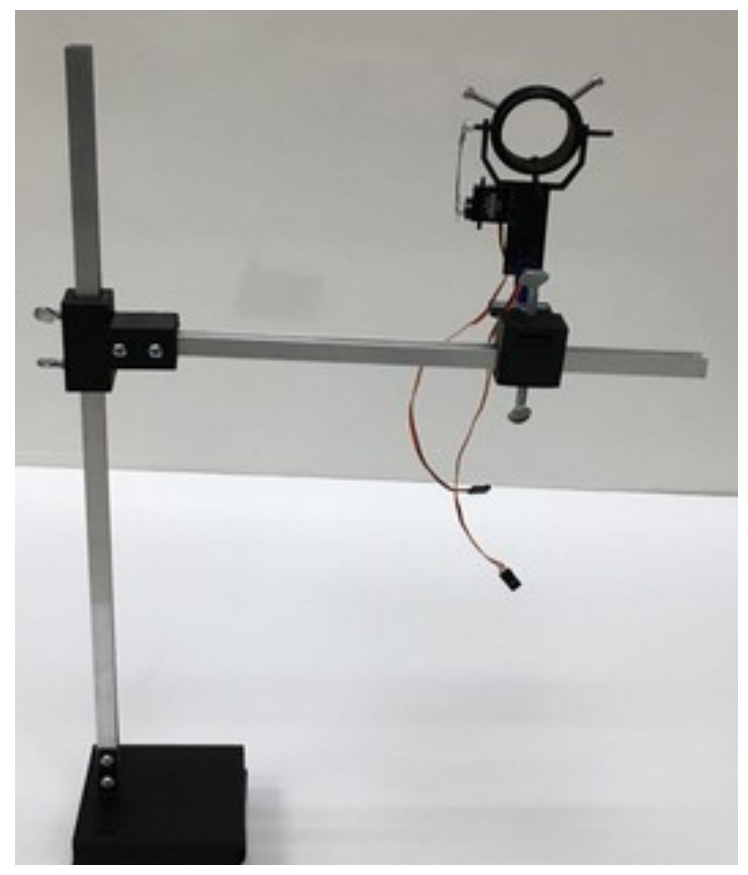

Figure 5-34: Full implemented OphthBot 2 lens holder

\subsubsection{Complete OphthBot 2 Implementation}

The two parts of the second system were integrated into one unit. The same control system and user interface was used for both OphthBot prototypes. A camera mount was designed and implemented to provide visual feedback via the web control interface. The complete implemented OphthBot 2 system is shown in Figure 5-35. Further details on how both prototypes functioned and compared will be provided in Chapter 8 . 


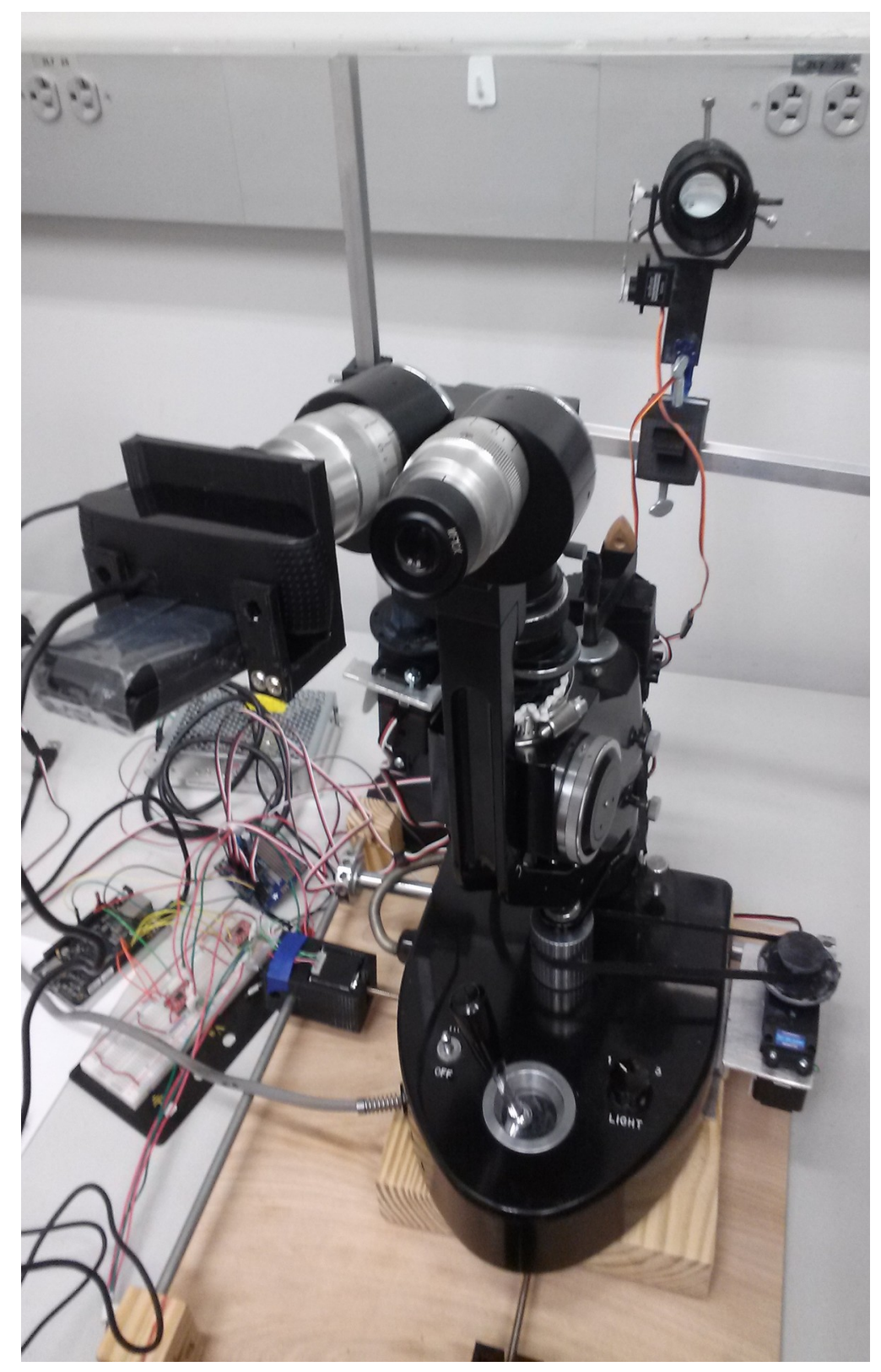

Figure 5-35: Complete OphthBot 2 system 


\section{REHABILITATION ROBOTS DESIGN AND CONSTRUCTION}

This chapter provides an overview of three robots that were designed and produced that can be used as models for aiding in physical rehabilitation, among other purposes. They also form a continuum with the ophthalmic project and reiterate the interconnections that robotics allow amoung medical fields.

These projects were in a large part completed with the participation teams of undergraduate with the initial ideas, concepts, and guidance provided by the author of this work. The undergraduate teams, however, performed nearly all of the detailed design and all of the construction of the projects presented in this chapter. The control systems are similar to those used in OphthBot and notes are provided about this as well.

\subsection{Robotic Face}

The first of the projects directly bridges eye procedures to rehabilitative uses. It can also be used in other fields and interest areas of robotics including creating more life-like androids or social robotics. The primary focus of this project was to develop realistic robotic eyes, though provisions were made for actuation of the complete face. 


\subsubsection{Goals of the Robotic Face}

The primary goal of this project was to design and implement a platform for which to test devices such as OphthBot as well as to allow for a means to train physicians for performing non-robotic ophthalmic procedures with animate eyes. While it is possible to perform these tests on animals and the goal is not to completely replace this practice, animal testing is expensive and when used extensively it raises concerns for some individuals and organizations. In addition, animals are not exactly like humans and the exposure to all situations and conditions for which a physician should be trained are not available. A robotic platform could be reused over and over again and can set-up to simulate various eye diseases and conditions. As with current training, artificial eyes with replaceable retina films can be used to provide practice on laser techniques in a simulated in vivo environment with no patient in danger if mistakes are made.

The secondary goal of this project is to serve as a model face to help in rehabilitation tasks. Most notably is for use in teaching or expressing sign language. American Sign Language (ASL) not only uses hand shapes and movements, but facial expressions and body language as well [107]. In order to fully model ASL and other dialects of visual communication, realistic facial expressions are needed. This aspect of the project will tie into the project discussed in Section 6.2 and more details will be provided there. 


\subsubsection{Robotic Face Implementation}

The robotic face was successfully produced with moving eyes, though the mouth and other features were not completed in this iteration. Details on the design choices and manufacturing of this project have been published elsewhere [93] and are not critical to this work to be fully repeated here. The resultant prototype had individually-actuated eyes controlled by servos with wire linkages, somewhat like the tendons and muscles in biological human eyes. This provided a realistic model complete with the ability to shift gaze and blink lids. The prototype is shown in Figure 6-1 below.
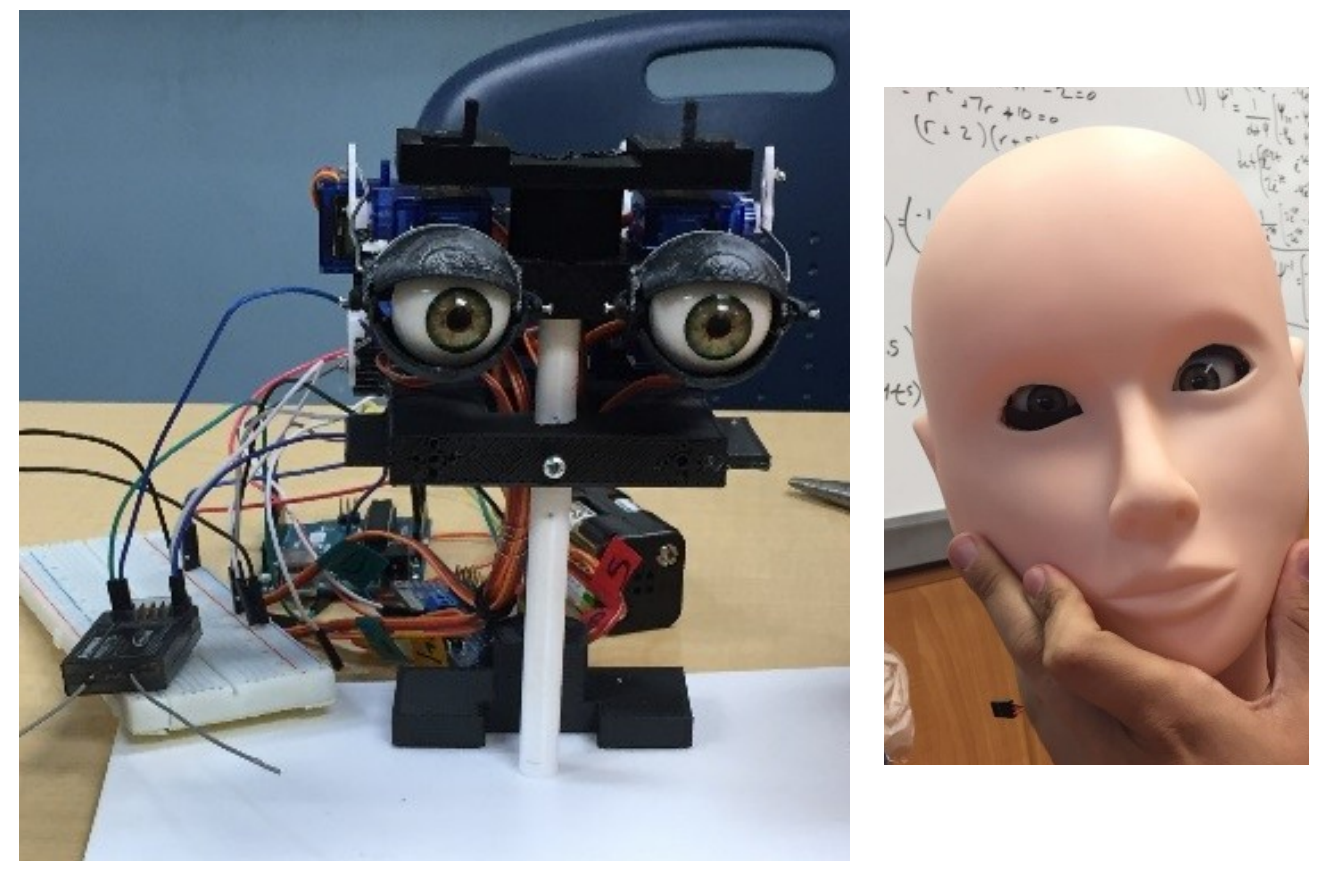

Figure 6-1: Robotic face without cover (left) and with cover (right) 


\subsection{Robotic Arm and Hand}

The next progression from the robotic face is to include the hands and arms. A realistic robotic face can be used for medical simulation, but also provides an important component for making a realistic humanoid model for other tasks. As with all projects in this chapter, more details on the design and implementation are documented elsewhere [66].

\subsubsection{Goals for Robotic Arm and Hand}

The primary goal for the robotic hand begin with the secondary goal of the face; to be a model for ASL. A robot that can model any sign language would also be able to produce it as a translation of spoken or written communications in any language. This can be useful in situations were a human translator is not available, or when a crowd is too big and having another sign language translator would be useful.

A robot that can model sign language is not a solution for all needs. Most adults can learn ASL without a physical model such as when using videos. Many children are able to learn this way as well. However, there is a lack of depth when learning ASL from videos. A physical model can allow the student to change angles and clear up confusion. A human teacher may not be available at all times, or could teleoperate the robot in order to reduce travel time and costs. This opens up on-site ASL instruction to individuals who may not be near instructors and want a more realistic learning experience. A robotic 
model programmed to perform signs can perform the same movements precisely over and over again and do so at different speeds without changes in positions as well. All of this can be done on-demand as needed. Finally, it can provide a tactile experience in learning that videos can not provide. This can be helpful to some learners or audiences, especially those with limited vision.

This robot project had a secondary goal as well. This goal is to provide a model for movements in many physical rehabilitation activities. The fully articulated arms, hands and fingers allow for use modeling many occupational therapy tasks as well.

\subsubsection{Project Implementation}

The project was based primarily on the InMoov open source project mentioned previously [88], though alterations were necessary. Alterations were made due to inaccuracies of the $3 \mathrm{D}$ printer utilized. In addition, adaptations were made to allow for slightly different servo motors that were used based on availability and costs. The revisions were extensive time-wise due to the large size as each individual set of parts took well over three hours to print. Due primarily to this fact, only one arm was completed in this phase of the project. The result is shown in Figure 6-2 below. 


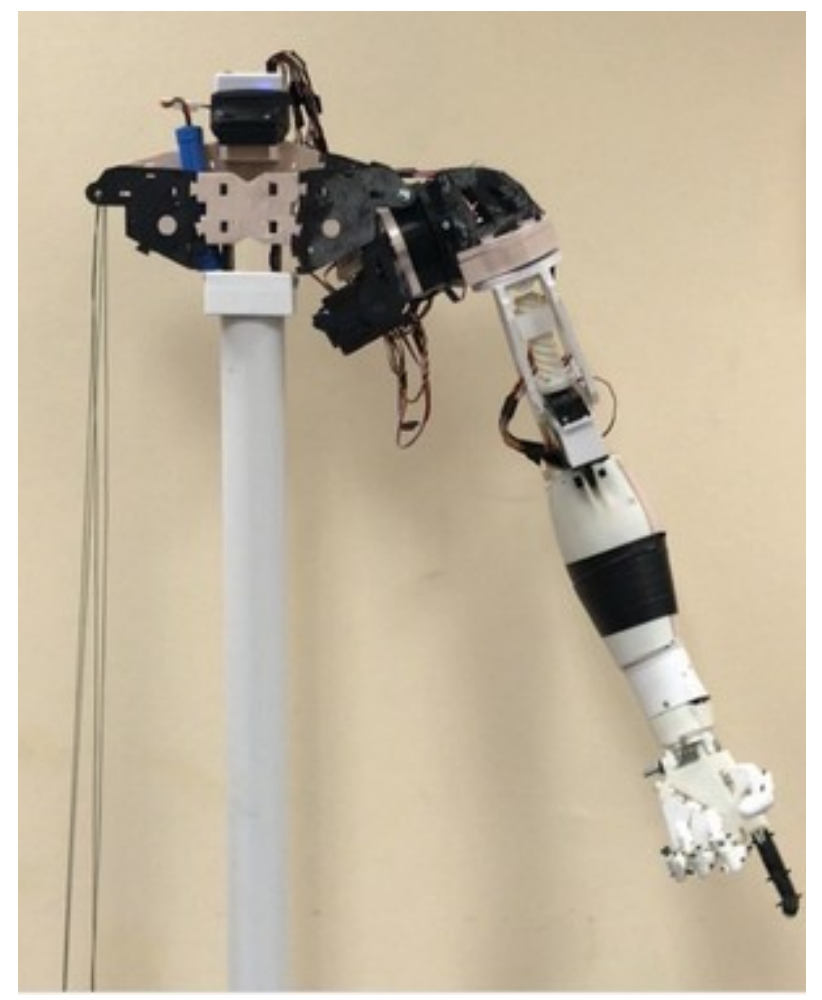

Figure 6-2: Robotic hand as completed

The project had some other minor adaptations as well. The index finger was printed in a different color to help facilitate the identification of the hand and finger positions when making various gestures. Other fingers could be made in different colors to further differentiate them.

Though it was not implemented in this phase of the project, the 3D printed open source base allows for other adjustments to be made. Like the humanoid project that will be discussed later in this chapter, the arms and fingers can be modified to represent the actual configuration of an individual. The adaptations would allow the robot to perform any occupational therapy task, including sign 
language, accurately modeled given each individual client's ability and physiology. The size and proportion of the arm, hand and fingers can be adjusted as well as the form and number of fingers. Servo motors can be controlled in such a way to mimic any difference in strength or range of motion.

\subsubsection{Control System}

There were two control systems implemented in this project. The first uses the base system of control used in the ophthalmic project. The second utilized an all-in-one controller. It allowed for some direct comparisons in controller selections.

The first controller utilized was a Raspberry $\mathrm{Pi}$ along with the Adafruit servo controller used in the ophthalmic project. The program was written in Python as in OphthBot as well. This did allow for full control of the robot and some progress was made. However, programming was step-by-step and individual for each joint motion. This made programming tedious.

Toward the end of the time-frame of this project, an EZ-B version 4 by EZRobot, Inc. was implemented as a control system replacement. This device is shown in Figure 6-3. 


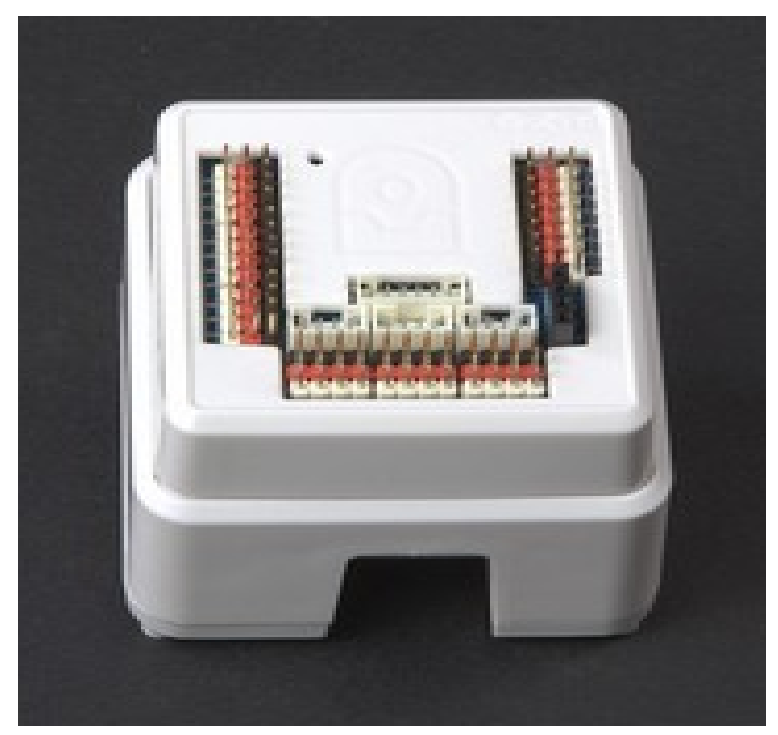

Figure 6-3: EZ-B v4 servo controller

The EZ-B allowed for built-in WiFi capabilities and serves a webpage for control. It can control 73 servos, which was perfect for the servo-intensive device. It was also able to control all three types of servos used in the project as there were various ones selected due to functionality and costs constraints.

The EZ-B was programmed using MyRobotLab, which allowed for gestures to be created simply by picking end position needed. The software performed the kinematic calculations and greatly simplified the task of programming the robot. Several signs were programmed in this phase of the project to complete the proof-of-concept and meeting the minimal goals. Further work will be done completing both arms and further developing the tools and programming to make the system fully meet the goals outlined. 


\subsection{Humanoid Robot}

In the final stop in the progression of projects, a full humanoid model was investigated. This robot, again published in more detail in other publications including [85], is a full humanoid scaled-down model to perform motion research as well as modeling tasks. It was named HCTeR for Humanoid Companion Technology for Rehabilitation.

\subsubsection{Humanoid Project Goals}

Humanoid robots have several uses in therapy. They can be used to study motion dynamics in real life without tiring or endangering humans. Mostly, this is important when trying to develop motion planning for an impairment that may not be well understood. Still, computer models and working with the patient to determine best courses of action are sufficient and provide a more realistic dynamic model given the size and costs limitations of this project. Due to these factors, this isn't a primary goal of this particular humanoid model.

One goal that is considered for this project is to allow the robot to act as a surrogate between the therapist and the child. This is important in cases such as the child being on the autism spectrum, where direct adult-to-child interactions may be difficult or impossible. However, the therapist could control the humanoid robot and elicit interaction via this device. A major objective of this robot was to be able to model physical therapy tasks in order to achieve the goal of being a surrogate. A secondary objective inline with this goal was 
for the robot to be able to help develop a child's social skills. This allows for a more holistic therapeutic tool to help the child gain the maximum benefit from all therapies as well as in life.

As already mentioned in this work, robotic systems such as HCTeR allow for customization of the limb proportions and strengths so that the robot can better model a task as the impaired patient would be able to realistically mimic. This was the primary goal when the project began, though it became the secondary goal as things developed due to a greater prevalence of autism and the urgent call for support tools. However, the goal of making a customized and adaptable system for each individual is unique to this system as far as the literature review revealed. Many children, and even adults, have different body proportions or configurations from that which is considered typical. Examples include shortened limbs in some individuals with Down Syndrome, reduced limb size or strength in children with muscular disorders, impairments to only one side in victims of stroke or certain head injuries, and altered or missing limbs due to birth defects or amputations. This is the only system with the objectives of being so inexpensive and easily manufactured, that it can achieve the goal of being made individually and to the specifications for each patient.

\subsubsection{HCTeR Implementation}

The robot was first modeled to closely follow the Poppy Project mentioned in the literature survey. However, it was never intended to fully follow the 
Poppy design and components. The first immediate designs focused on finding ways to adapt the Poppy design to accommodate lower-cost servos and control components. The Poppy Project as it currently stands costs nearly ten thousand dollars, which is not a reasonable amount to allow for individuals to afford the system. Furthermore, the higher cost components, while allowing for some great features, also increase the cost of repair should a motor be damaged during use as can be expected with repeated use around children.

Ultimately, the design differed fairly drastically from that of Poppy as can be seen from Figure 6-4 below. The body is more enclosed than that of Poppy, shown in Figure 3-17, which keeps components and wires out of sight and out of grasps of children. HCTeR is also much less intimidating in appearance than Poppy due to the enclosed nature and the more realistically shaped head and torso. Clearly visible, separate and expressive electronic eyes further add aesthetic appeal to HCTeR.

HCTeR differed from Poppy in other ways as well. The degrees-of-freedom were reduced from Poppy's 21 to only 18 in HCTeR. This cut costs, but mostly simplified the design where the extra motions did not aid in the goals of the project. In addition, low-cost hobby servos including the TowerPro HG996r standard servo and SG90 micro servo were used in place of the much more costly Dynamixel servos specified for Poppy; This alone cut thousands of dollars in costs. Less expensive control hardware was paired with the servos using an Arduino and the Adafruit servo driver used in the ophthalmic project. 


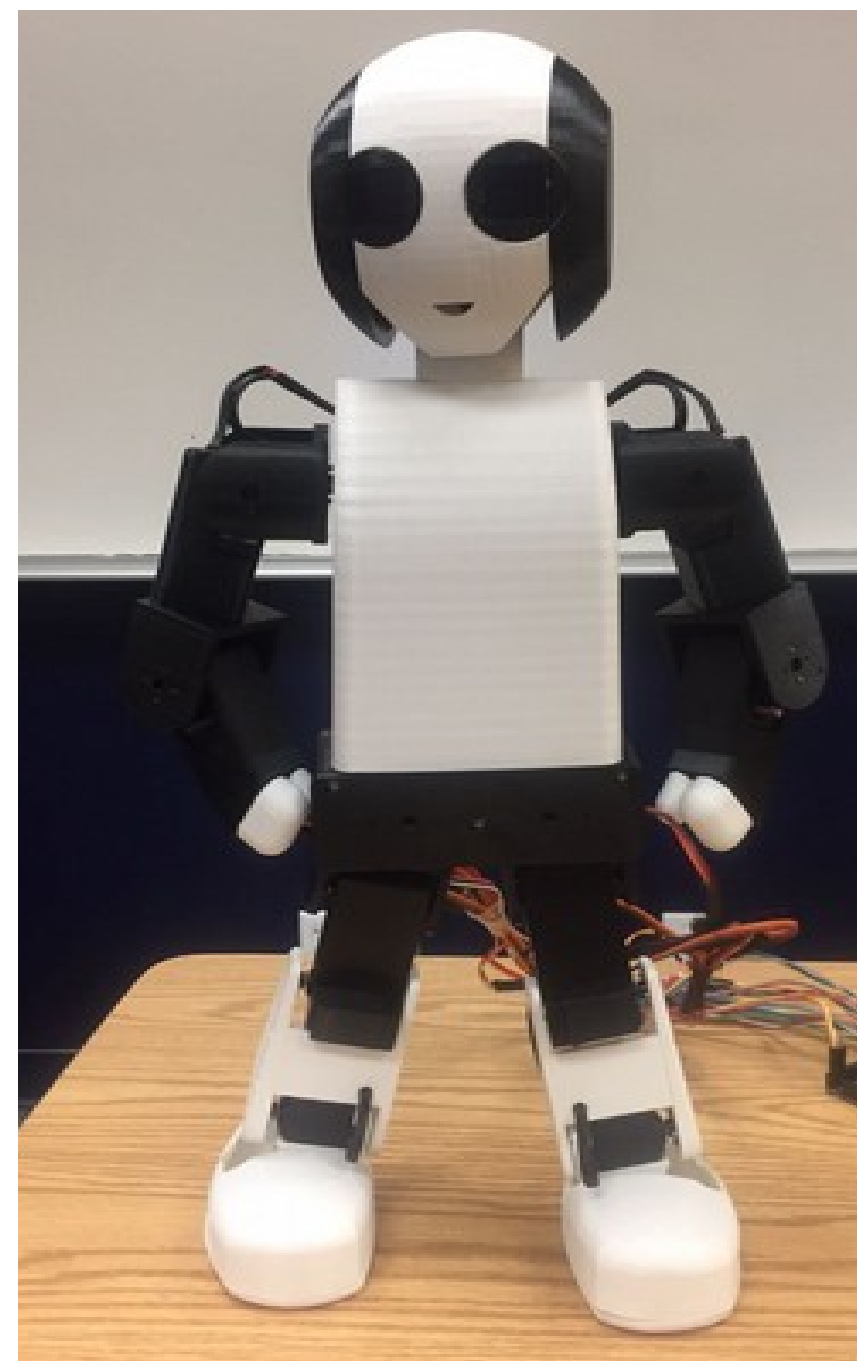

Figure 6-4: HCTeR Humanoid Rehabilitation Robot

The resulting project performed tasks such as crawling and would walk assisted with its hands held, accurately modeling these tasks for a child to mimic. Time did not allow for customization with various proportioned limbs, however. This, with the integration of sensors, addition of force feedback, and the implementation of internal battery power is left for future work. 


\subsection{Therapy Robots Summary}

The three robots described in this chapter all demonstrate how inexpensive robot technology can be used to help aid in therapies, especially those for children. They have other uses outside of therapy as well as they can be used as surrogates for humans in training doctors, be teleoperated in remote environments for safety or convenience, and be used to interact with those on the autism spectrum.

Future work with these platforms include adding more sensors and capabilities to the devices. More programming and motion development is also required on all of these systems. Finally, testing and development alongside physicians, therapists, and clients is a final step to verify that these projects fully achieve their ultimate goals. 


\section{CONTROLLER DESIGN}

This chapter explores the design of control systems for use in the prototypes, particularly that of the ophthalmic project. This includes the modeling and compensation methods for the major actuated components of the systems. The controllers were expected to not only be useful in improving functionality of ophthalmic and rehabilitative systems, but also small-scale manufacturing and systems developed by students and hobbyists influenced by the Maker Movement. Limitations of this work are noted.

\subsection{Control System Requirements}

The requirements for the control system in the scope of this complete work are varied as there are different actuators used, applications in which the systems are applied, and approaches taken. There are, however, some fundamental criteria that are the same. Further, to simplify and restrict the scope of this work, hobby and robotic rotational servo actuators are the primary focus. In addition, some consideration for small stepper motors were made.

In looking at the criteria of the control system, cost is a main consideration. Given the goal of making surgical, examination and rehabilitation robotic devices that can be used in numerous remote locations, the hardware used must be made as inexpensive as possible. This would enable small clinics, 
clinics in rural areas, and facilities in economically depressed areas and developing countries to have a greater chance of obtaining the on-site devices. It would also aid in making the systems affordable for in-home use in the case of rehabilitation and some diagnostic instances whether or not insurance covered the devices. Finally, since the scope of this work also includes consideration that devices that are accessible to the general public including students and hobbyists influenced by the Maker Movement, the controller costs must be kept down so that it is in-line with the expectations and limitations of this population.

Next, the controller will be used in a system that has inherent time-delays. These time-delays are due to the lag in control over the Internet or any future remote communication system that may be implemented. This time-delay must not only be tolerated by the controller and not cause further disturbances or inaccuracy, but ideally the controller may even offer some compensation. An example of this would be to limit the reaction of the device in response to a quick series of commands given over the remote connection. The inherent delay may cause the remote operator to inadvertently cause an overshoot in motion by sending a command too many times. In any case, the controller should not enhance the overshoot that the operator may have implemented.

Robustness is critical for a controller that is being used for a robot in direct contact with human beings. Human beings can behave erratically and unpredictably at times, particularly children at play. Though the devices will 
ideally be used within a clear range of expected conditions, the system should perform reasonably well and have a relatively predictable response to conditions that may be outside of the normal modes. In some instances, the response may be to get to a shut-down mode as quickly and safely as possible. Unexpected conditions can include another person interacting with the device, the patient disregarding directions and applying excessive forces, a patient trying to move the system in a way that was not intended, a patient falling or becoming incapacitated while using the device, a component or appendage being altered while in use or rest, a child or pet stepping in the way, etc. The system should have a controller that is stable in these conditions and is able to at least maintain safe motion control. The device should never move too quickly or too far such that it could cause injury to the patient.

The final major criterion for the control system is that of adapting to not fullyknown, or in some cases mostly unknown, model variables. In the case of a ground-up designed robotic slit-lamp or new medical device, the model will be well known and a controller can be made accordingly. However, there are a lot of used slit-lamps and medical devices that could be retrofitted for robotic use and thus lower the upfront costs for implementation. The controller in such a retrofit kit would be designed for a known model of a similar system, but it could differ in a number of ways. Similarly, a controller developed for a pediatric rehabilitation system should be able to work with a range of unknown factors. This would enable a device to be used as-is without further programming and 
modification by the users in children of a range of sizes and abilities, though these ranges could be specified and limited.

In summary, the control system must meet several main criteria as follows:

- Use low-cost hardware to facilitate adoption of implemented systems

- Tolerate time-delays that may be inherent in the controlled systems

- Exhibit robustness in the presence of unexpected conditions

- Adapt to new and not fully-known/defined models

\subsection{Modeling}

Modeling is an important first step in developing a control system. As noted above, the exact model may not be known for all of the proposed applications. However, a general idea of the model is known for all cases. In addition, the range of conditions is known or can be specified for each application. Models are developed for the slit-lamp, lens holder and for possible motors used in this project.

\subsubsection{Slit-Lamp Model}

Due to the prototype being a robotic retrofit, the exact specifications of the slit-lamp were not known. In this case, full disassembly of the slit-lamp was not possible as many parts were permanently adhered and the system was not meant to be fully serviceable. Accurate spatial measurements were feasible as 
were the range of motions. The exact weights of components were not known, though these can be closely estimated and forces needed for motions were able to be measured as well.

To simplify the system, the slit-lamp model was comprised of several smaller models, one for each joint. These sub-models can then be individually controlled by a control system designed for that joint. A controller was then implemented to handle the entire range of conditions expressed by all of these joints.

Another consideration in the dynamics of the model was that the robotic slitlamp itself should never move very quickly. This was for safety reasons rather than technical limitations as the actuators allowed for quick motions. The slitlamp needed to move slowly to prohibit it from hitting a person or object with notable force. This reduces the chance of injury or damage in the event that the remote operator is unable to detect that something is in the path of motion. It also limits the overshoot that may be caused due to the latency when controlling the robot remotely. A slower motion with smaller increments will cause more gentile motions should a command be given more than once in the event that the operator does not see a reaction as quickly as expected and hits the command again. 


\subsubsection{Base Platform}

The first aspect of the slit-lamp to be considered is the motion of the base platform. This platform only moves in a plane and is constrained by a frame consisting of rails on both sides. This portion of the slit-lamp was modified by raising it and placing it on Omni-wheels in the case of OphthBot 1. The wheeled platform is therefore the main aspect to be modeled for this motion.

Simplistically, it can be modeled as a platform with simple wheels. It does vary from this scenario because the wheels are not positioned as in normal vehicles, but it can be shown that this has limited to no effect.

Research on previous platforms using Omni-wheels have shown wheels to be aligned evenly around the outside or in a symmetrical pattern underneath the platform [118] [84] [82]. In the case of OphthBot 1, however, the wheels are mounted in a T-shape as shown below.
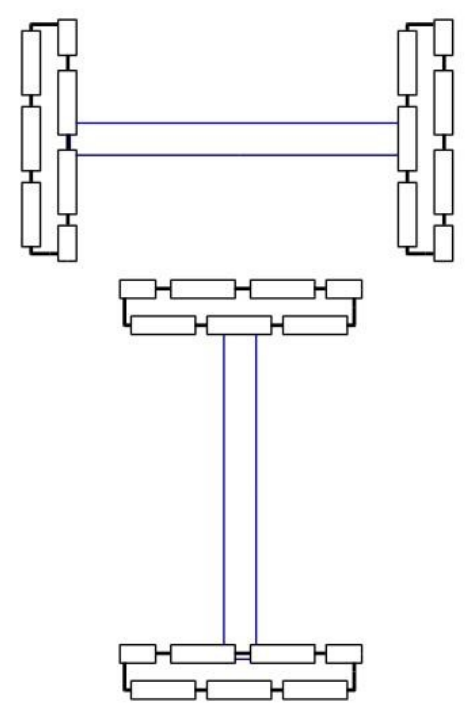

Figure 7-1: Configuration of wheels and axles in base platform of OphthBot 1 
Typically, the position of a mobile robotic platform in a plane is described using the following rotation matrix and translation vector:

$$
\begin{array}{lll}
R(\theta) & =\left[\begin{array}{ccc}
\cos \theta & \sin \theta & 0 \\
-\sin \theta & \cos \theta & 0 \\
0 & 0 & 0
\end{array}\right] & \text { Equation 7-1 } \\
T=\left[\begin{array}{l}
x \\
y \\
\theta
\end{array}\right] & & \\
& &
\end{array}
$$

However, in this case, the angle and rotation can not change as the base platform is bound by the track system of the slit-lamp. Therefore, the position is only the translation in a plane. It reduces the position to a simple vector:

$$
P_{\text {Wheeled Base }}=\left[\begin{array}{l}
x \\
y
\end{array}\right]
$$

In addition to the translational motions allowed by the base, there is an adjustment just above the base that allows for the slit-lamp assembly to be raised and lowered. As it moves everything but the wheeled platform, it is convenient to include it is the base kinematics to keep the number of equations more compact. Therefore, the full base position vector becomes: 


$$
P_{\text {Base }}=\left[\begin{array}{l}
x \\
y \\
z
\end{array}\right]
$$

Equation 7-4

A more complex model can be developed considering that it is not simple wheels that are used, but instead Omni-wheels. This changes the dynamics of control and motion due to their geometry and properties. There is slippage and other factors that can be considered in some cases [118] [19]. However, the models used in these works included the use of re-purposed cargo rollers in one case and the rotation of casters in another, neither of which matches this example. In fact, no examples found in literature indicated that there were special considerations for dual row Omni-wheels.

Based on single-row wheels, it was assumed that the main factor with these wheels is the uneven motion they experience when rolling along the activated hub plane (the typical direction of motion expected of a wheel) and the slippage that results. This problem plagues the single-row designs is is a primary investigation in the literature.

A CAD model and tests with the actual wheels were performed. Four-inch diameter VEX Omni-wheels were used. As indicated in both the CAD model and photos, shown in Figure 7-2, the wheels are designed to be perfectly round in profile. The rollers are offset such that the wheel is always resting on at least one roller surface and never dips or slips due to the gap between rollers. 


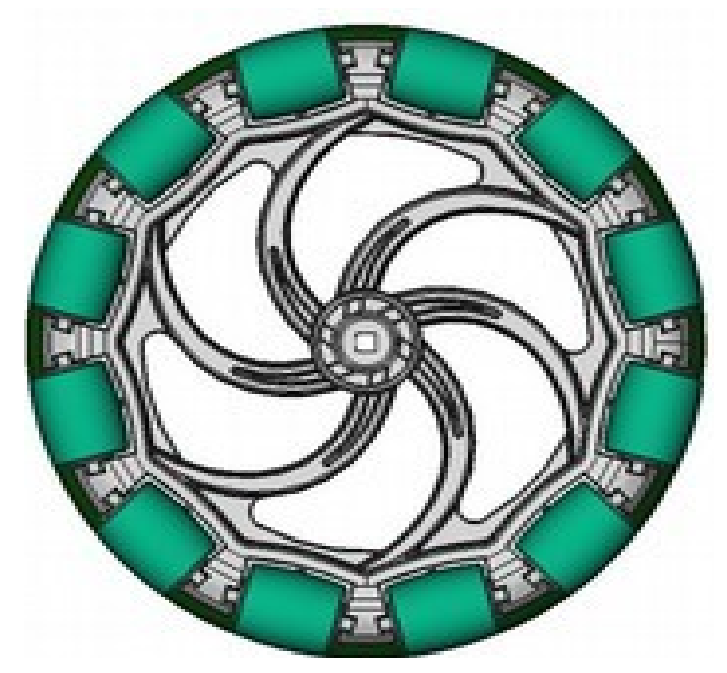

Figure 7-2: Dual-row Omni-wheel in profile

In practice, there was some minute dipping of the wheel in use due to movement and deformation of the rollers, but it was barely notable and certainly did not affect the positioning of the slit-lamp. Given the slow motions and relatively limited range of motion of this project, any effects of the Omniwheels in either plane of motion were not a major consideration for this project and were not investigated further.

\subsubsection{Rotational Arms}

The arms have a few aspects that are relevant to model. In addition to completing the model itself for each arm, there is also the aspect that rotation of the lower binocular arm will rotate the upper lamp arm. This requires some compensation so that the binocular arm can be rotated without undesired changes in the angle of the light. 
Looking at each arm individually, kinematic models can be made based on the geometry and range of motion. The main kinematic consideration for the arms is their rotational position in relation to the base in the X-Y plane. This is shown in Figure 7-3 where $M$ represents the microscope-binocular arm and $L$ represents the lamp arm.
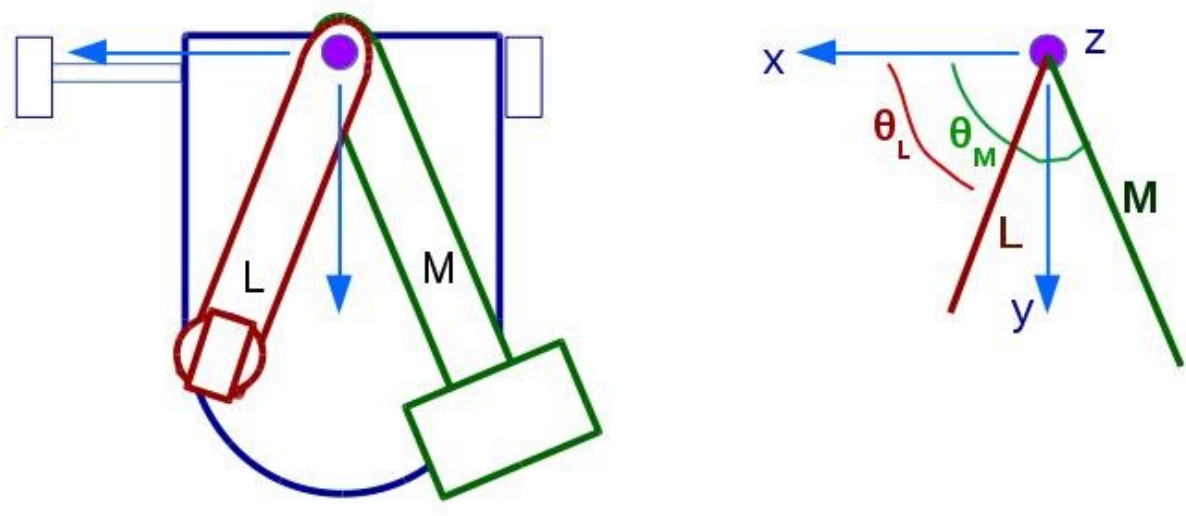

Figure 7-3: Kinematic model of slit-lamp arm positions

Given this diagram, determining the equations for motion of the arms based on the input angles of the servos can be found to be:

$$
P_{\text {Lamp Arm }}=\left[\begin{array}{c}
L \sin \theta_{L} \\
L \cos \theta_{L} \\
z_{L}
\end{array}\right]
$$




$$
P_{\text {Microscope }}=\left[\begin{array}{c}
M \sin \theta_{M} \\
M \cos \theta_{M} \\
Z_{M}
\end{array}\right]
$$

where $Z_{L}$ and $Z_{M}$ are known fixed offsets and $L$ and $M$ are known fixed lengths.

Coupling the motion of the lower arm with the top, the top arm needs to have corrective motions for every motion made on the bottom arm. The motion is simple in that as the lower microscope arm rotates, the lamp arm moves directly with it. In order for the lamp to remain in the same position, it simply must be rotated in the opposite direction when the microscope arm is moved. In other words, for every $\theta_{M}$, there is a $-\theta_{L}$ commanded. This is directly programmed as a joint motion when programming motions of the microscope arm.

\subsubsection{Control Knobs}

The control knobs are automated in retrofit form by using friction fit discs, belts or gears to connect the servos. This means that the knobs are modeled as physical knobs as they are not digitized or manipulated in an alternative manner as may be done if the slit-lamp were custom-designed to be robotic from the start.

A model can be derived for each knob based on the diameter. It actually involves the calculation of gear ratios whether an actual gear or disc is used 
and is simply the driver (servo horn) circumference over the driven (knob) circumference, both of which are known and fix values:

$$
\theta_{\text {knob }}=\frac{\pi d_{\text {servo }}}{\pi d_{\text {knob }}} \theta_{\text {servo }}=\frac{d_{\text {servo }}}{d_{\text {knob }}} \theta_{\text {servo }} \quad \text { Equation 7-8 }
$$

Each knob can therefore be modeled using this equation. Substituting the diameters of both the knob and the driving actuator to determine the position of the knob given the position of the actuator.

\subsubsection{Model Limitations}

There are some limitations of the models that should be noted. These include assumptions, simplifications and details that could not be determined precisely.

The first major limitations of the model is that it ignores the effects of the position of the binocular and lamp arms in the overall inertia of the system. In reality, the positions of these arms does change the center of mass and dynamic characteristics of the system, particularly base motions. It is ignored, however, due to the fact that the motions are so small and slow that these changes do not have a significant role. If the system were to move faster, however, these would have to be taken into account. The inverse would also be a concern as well. In the case of large or fast base movements, the 
binocular and lamp arms may attempt to rotate. In these instances, the model would need to show this and their affects so that compensation can be provided for both the base motions and to stabilize the arms. Again, the slow and incremental motions of this system do not make this a considerable factor.

Another limitation is that all friction in the slit-lamp is not taken into consideration. Some friction was considered when measurements were taken from the actual slit-lamp to build the model or controller. However, it was omitted to simplify the project.

\subsubsection{Lens Holder Model}

The modeling of this component of the project differs drastically from the main slit-lamp portion in a couple of ways. First, the mechanism is much smaller and lighter. Second, the motions may not be as slow, though the full speed was not used in this particular prototype. Though motions may be incremental as with the slit-lamp, some motions may be made automatic so that the lens tracks and stays firmly against the eye.

The model provided here is for the lens holder designed alongside Ophthbot 2 as it was the most robust and complete lens system. This lens holder had multiple stages of motion in which to model. As with the slit-lamp, the equations for programming motions will be noted from the base upwards.

The kinematics of the lens holder base is represented below in Figure 7-4 with equations for determining the position noted immediately thereafter. 


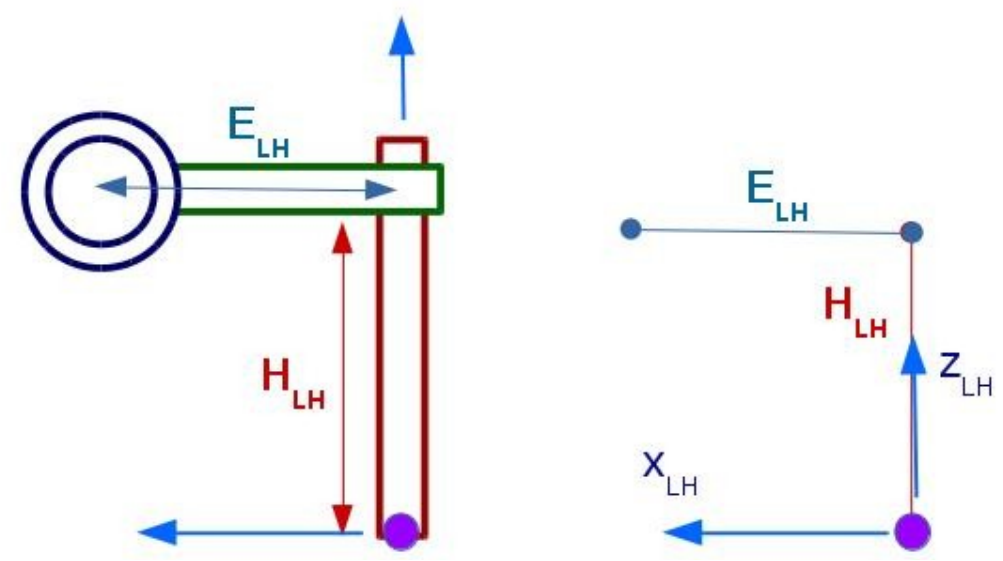

Figure 7-4: Kinematic model of the lens holder base

$$
P_{\text {LensBase }}=\left[\begin{array}{c}
E_{(L H)} \\
-D_{(L H)} \\
H_{(L H)}
\end{array}\right]
$$

Next, the position of the lens bezel is considered. There are several partial rotational motions, a full rotational motion, and two offsets to be considered. First, consider the vertical and horizontal partial rotations of the bezel shown in Figures 7-5 and 7-6. Next, the rotation of the lens is shown in Figure 7-7, completing the actively controlled lens motions. The composite equation for the actively controlled lens motions is shown in Equation 7-10 below. 

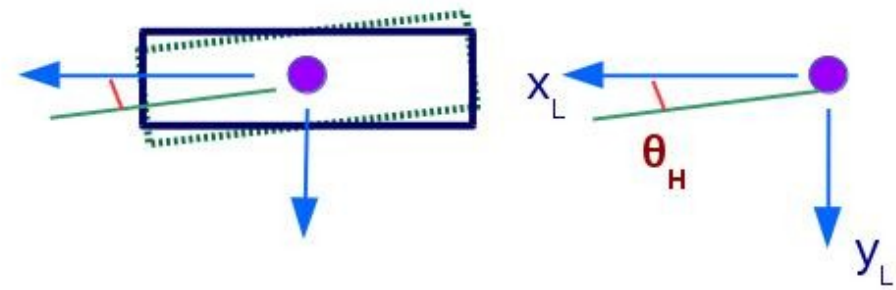

Figure 7-5: View from top of lens holder bezel - horizontal partial rotation

$Z_{L}$
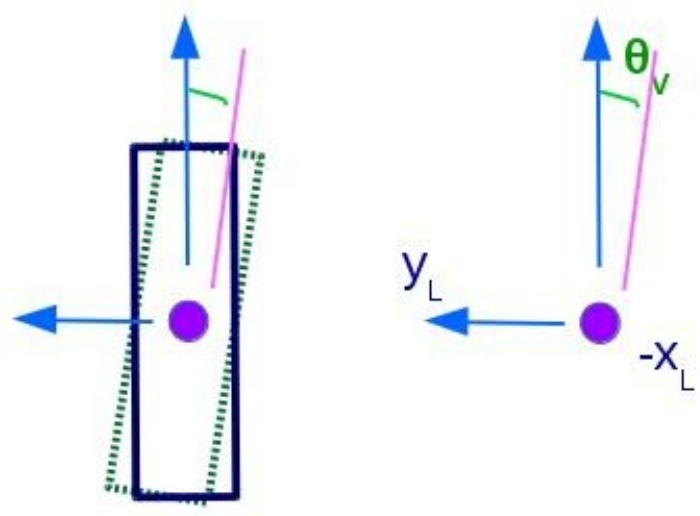

Figure 7-6: View from side of lens holder bezel - vertical partial rotation 

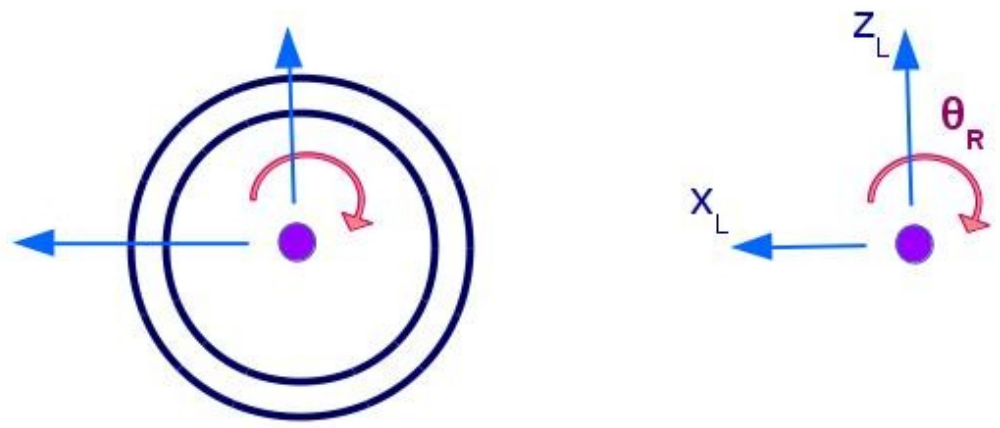

Figure 7-7: View from front of lens holder bezel - full lens rotation about the eye

$$
P_{\text {lens ActivelyControlled }}=\left[\begin{array}{c}
E_{(L H)} \\
-D_{(L H)} \\
H_{(L H)} \\
\theta_{V} \\
\theta_{R} \\
\theta_{H}
\end{array}\right]
$$

Two offsets are also possible pending the manual placement of the lens as well as variable lens parameters such as the lens depth. These are shown in Figures 7-8 and 7-9 below. A complete equation for the kinematics of the lens position for both actively controlled and user offset positions is shown in Equation 7-11. 

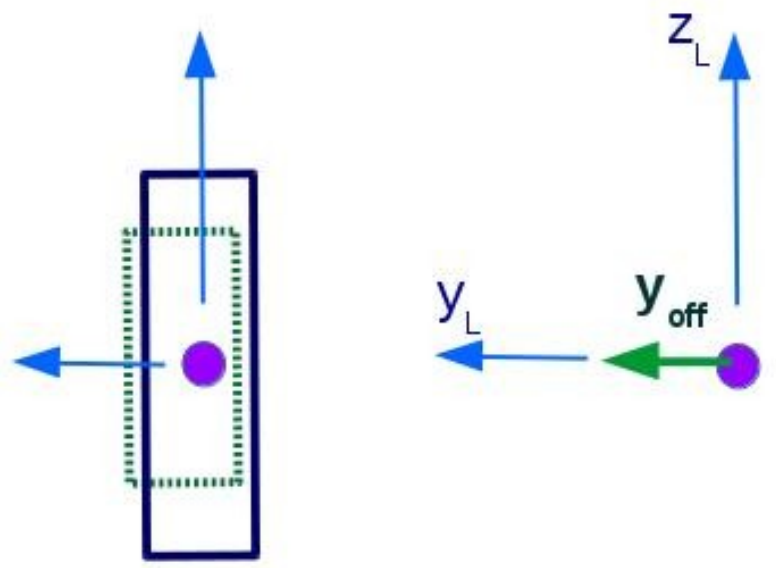

Figure 7-8: View from side of lens holder bezel - lens depth offset
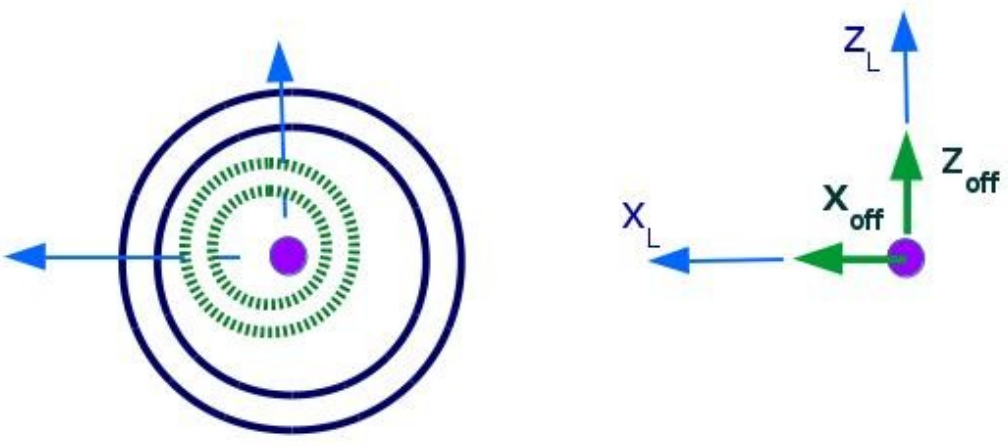

Figure 7-9: View from front of lens holder bezel - lens center offset

$$
P_{\text {Lens }}=\left[\begin{array}{c}
E_{L H}+x_{o f f} \\
-D_{(L H)}-y_{o f f} \\
H_{L H}+z_{o f f} \\
\theta_{V} \\
\theta_{R} \\
\theta_{H}
\end{array}\right]
$$




\subsection{Controller Design}

The methodologies selected for controlling the system must meet the main criteria provided at the beginning of the chapter. As it would be impossible to design and test every feasible control methodology for this system within the limits of this work, it was decided to select a couple of controllers. This provided means to test the possibilities of control algorithms on the hardware and systems presented.

The controllers were designed to be used with the systems provided, though the ophthalmic project was the primary focus given the time limitations for implementing models on the physical prototypes in this work. Designs were made for both the slit-lamp and the lens holder as they are both controlled remotely at the same time and must coordinate to allow the desired view and control of the eye exam or procedure.

Narrowing down all of the possible control methodologies to select those tested required consideration of the system, prototype goals, and potential knowledge gains of all of the possible candidates.

The first selected control methodology was the PID controller. This was chosen for a number of reasons including being the most widely-used control method. The PID controller is shown in Figure 4-3 shown previously. 
The equation for this controller shown in the diagram is:

$$
u(t)=K_{P} \cdot e(t)+K_{I} \cdot \int_{t_{0}}^{t} e(\tau)+\frac{K_{D} \cdot d}{d t} e(t) \quad \text { Equation } 7-12
$$

There are numerous ways of tuning the controller. In this case, the simplest method was to set all of the gains to zero and adjust them until obtaining the desired control. This was the method selected as it allowed for a quick implementation as it was planned to design and implement several different controllers for comparison.

\subsection{Controller Implementation}

The controller was implemented in Python on the BeagleBone Black and used to run the robotics servos of the slit-lamp. The pseudocode is shown below.

Set $\mathrm{KP}, \mathrm{KI}$ and $\mathrm{KD}$

Set dt as the time interval

Read in the current position and the desired position

PreviousError $=$ Error

Error $=$ Desired position - Current position 
IntegralError $+=$ Error $* d t$

DerivativeError $=($ Error - PreviousError $) / \mathrm{dt}$

$\mathrm{U}=(\mathrm{KP} *$ Error $)+(\mathrm{KI} *$ IntegralError $)+(\mathrm{KD} *$ DerivativeError $)$

Sleep for time dt

Even with all gains set to zero and the proportional gain slowly changed, it was immediately clear that it had no effect. Adjusting any of the gains to any extent had no noticeable effect. This was tested repeatedly as shown in the next chapter. The addition of filters made with passive electrical components were added to the controller as well, though there was no difference in the servo performance as well. Due to the lack of response in differing software and physical control systems, no further control systems were developed nor implemented for this project, though a MPC and PLC were prepared and planned. This is further discussed in the chapters that follow. 


\section{RESULTS}

The details of the results from the physical prototypes and implementing the controllers are shown in this chapter.

\subsection{Functionality of the OphthBot Prototypes}

The overall performance goal of the ophthalmic prototypes was to meet, if not exceed, the capabilities of the slit-lamp and lens holder motion with a human doctor physically present controlling these components in-person.

To test the devices, no human nor animal tissue was used both for safety and due to institutional restrictions. Instead, an anatomically correct plastic eye was used. The specific model was the Reti Eye Laser Practice Kit by Gulden Ophthalmics. This is shown in Figure 8-1.

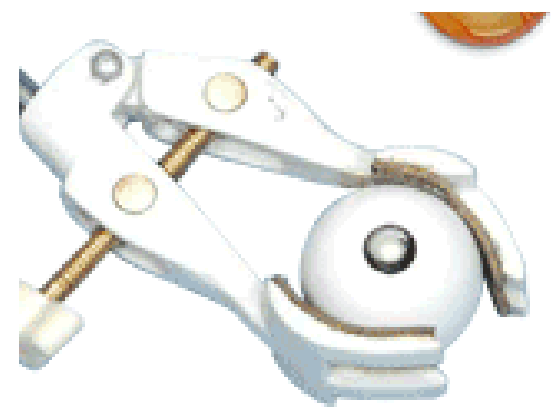

Figure 8-1: Reti Eye practice eye by Gulden Ophthalmics 
More detail on how the device performed both quantitatively and subjectively is provided in the next sections.

\subsubsection{Robotic Slit-Lamp Specifications}

The slit-lamp was designed and controlled to move with limited speed and range of motion, so the specifications provided are those of the controlled system and not the maximum capabilities of the actuators under the loads provided.

The first area tested extensively was the translational motions of the base of OphthBot 1. The testing and extensive use caused the realization that the base movements were the most troublesome and least accurate. Of the first prototype iterations, it was the only aspect that definitely did not meet the goals consistently.

The results from one series of tests of the OphthBot 1 base is shown in Table 8-1 below as it clearly demonstrates typical base behavior. It compares the distance that the base traveled after each incremental command in the two translational directions. It does this for both the unmodified controller running the software controller and a modified controller that had a passive filter to try to obtain a more controlled response. It is also clear from this data that the modified controller had no significant effect. Reasons for these outcomes will be discussed in the next chapter. 
Table 8-1: OphthBot 1 Base Movement Data (in mm)

\begin{tabular}{|c|c|c|c|c|}
\hline & \multicolumn{2}{|c|}{ Base Movements Unmodified } & \multicolumn{2}{|c|}{ Base Movements Modified } \\
\hline & Back and Forth & Left and Right & Back and Forth & Left and Right \\
\hline & Unmodified & Unmodified & Modified & Modified \\
\hline & 5 & 16 & 4 & 10 \\
\hline & 2 & 11 & 2 & 9 \\
\hline & 5 & 14 & 3 & 7 \\
\hline & 5 & 10 & 1 & 9 \\
\hline & 2 & 7 & 6 & 13 \\
\hline & 2 & 20 & 7 & 17 \\
\hline & 4 & 21 & 1 & 19 \\
\hline & 2 & 22 & 1 & 20 \\
\hline & 4 & & 7 & 7 \\
\hline & 1 & & 2 & 2 \\
\hline & 5 & & 6 & 6 \\
\hline & 6 & & 5 & 5 \\
\hline & 7 & & 6 & 6 \\
\hline & 4 & & 5 & 5 \\
\hline & 7 & & 2 & 2 \\
\hline & 3 & & 1 & 1 \\
\hline & & & & \\
\hline Average & 4 & 15.125 & 3.6875 & 13 \\
\hline Standard Deviation & 1.8618986725 & 5.5661605131 & 2.3012677665 & \begin{tabular}{|l|l|}
5.0426750271 \\
\end{tabular} \\
\hline
\end{tabular}

Similar data was obtained from all joints of OphthBot 1. As there was no significant difference with any of the attempts to improve or modify the controller, data was only fully collected using the original unmodified controller. Each actuator was given an identical step command so that the movements could be compared equally from that standpoint. The filter knob was not implemented due to space restrictions on the slit-lamp, so it was omitted from the practical tests. 
Table 8-2: OphthBot 1 Rotational Movement Summary (in degrees)

\begin{tabular}{|l|r|r|}
\hline & Average & Standard Deviation \\
\hline Microscope Arm & 5.75 & 1.82 \\
\hline Lamp Arm & 5.83 & 1.64 \\
\hline Height Knob & 1.83 & 0.71 \\
\hline Width Knob & 3.91 & 0.99 \\
\hline Chin Rest Knob & 3.33 & 1.07 \\
\hline
\end{tabular}

The table above shows clearly that the rotational arms have the largest rotational motions to a given increment command as well as the least amount of consistency in the motion that is produced. The arms show similar motions, which is expected as they have the same drive mechanism implemented as well as similar dynamic characteristics.

The height knob was the best performing mechanism of the prototype. It had both the best accuracy and precision. It also did not require any adjustment or further alterations during the months of testings.

The lamp width knob and the chin rest knob both performed similarly. This was expected as they had similar size, friction, and load characteristics. They were also actuated using similar friction discs. Their performance was median of the group of knobs.

The performance of the rotational actuation of the slit-lamp are shown in Figure 8-2 below. It gives more details of the actual values obtained during the testing trial shown and provides a visual comparison of the performance of 
various components. Further information on why the actuation performed as shown will be provided in the next chapter.

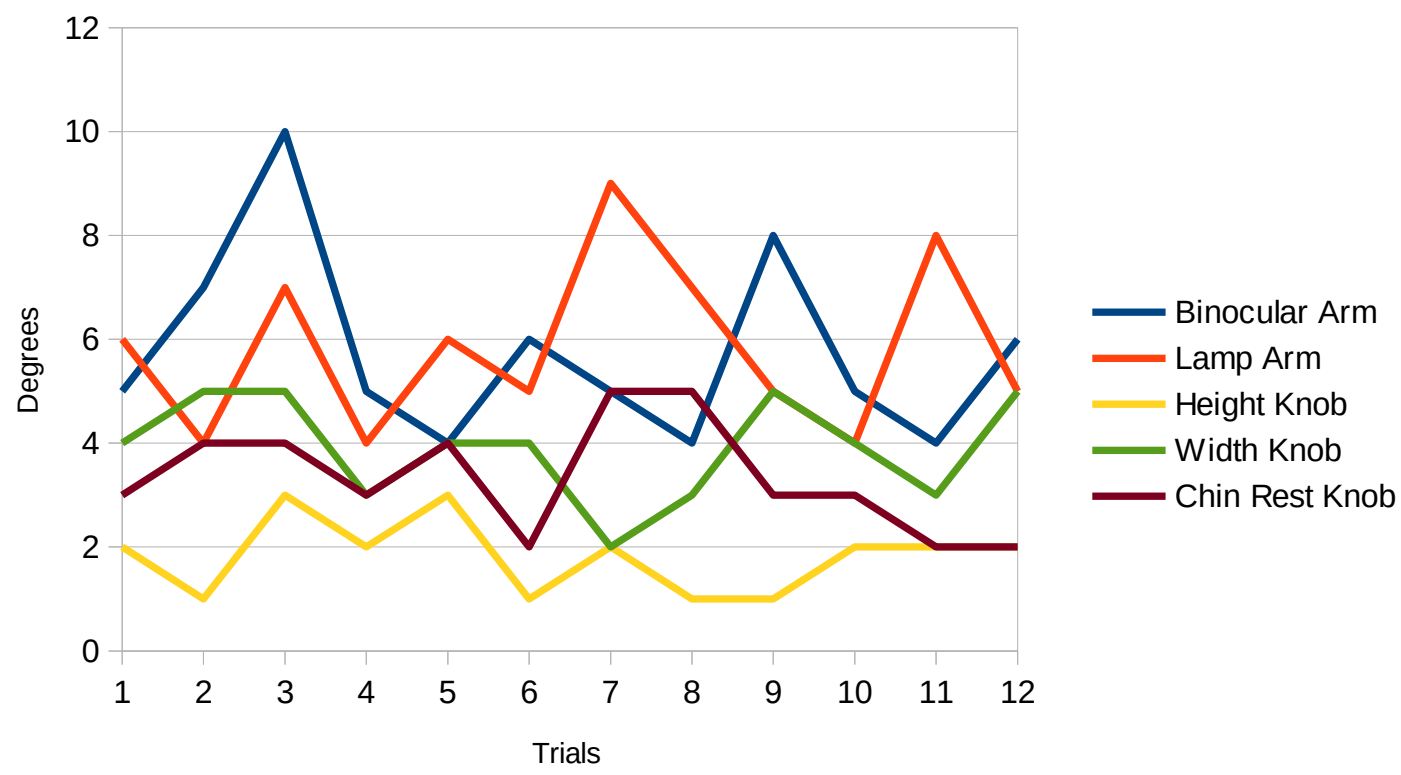

Figure 8-2: Graph of OphthBot 1 rotational component performance

OphthBot 2 did not have significant changes other than in the base movement mechanism. This is because the same actuators were used with only changes in how they interfaced with the joints. The connections were not necessarily as reliable as in the first prototype and so it was difficult to get a significant amount of trials before adjusting the joints. The small trials did not show any notable differences in data between the prototypes, however.

The X-Y table of OphthBot 2 was a huge change from the Omni-wheeled base of OphthBot 1, however. The X-Y table was not only consistent in both 
translational directions, but it had extremely consistent and small movements after each increment command. In fact, the increments were too small to accurately measure with the same instruments used with OphthBot 1 . Each increment was under one millimeter in linear motion. This will be discussed further in the next chapter.

Overall, the specifications for OphthBot 1 can be briefly summarized as:

- Linear movements in the X-direction: $15-\mathrm{mm} \pm 5.6-\mathrm{mm}$

- Linear movements in the Y-direction: 4-mm $\pm 1.9-\mathrm{mm}$

- Rotational movements of the microscope and lamp arms: $5.8^{\circ} \pm 1.8^{\circ}$

- Rotational movements of the lamp width and chin rest knobs: $3.9^{\circ} \pm 1.0^{\circ}$

- Rotational movement of the lamp height knob: $1.8^{\circ} \pm 0.7^{\circ}$

For OphthBot 2, the first two summary specifications can be replaced by:

- Linear movement of the $X-Y$ table: $<1-\mathrm{mm}$

\subsubsection{Lens Holder Specifications}

The lens holder used different servos and had slightly different results during testing. As with the testing shown above, it was done under the unmodified controller and using the same increments of motion as for all other components.

The three linear adjustments of the base of the lens holder were not required to be actuated by the project goals. In order to simplify the project and 
ensure completion by the deadline, these joints were never roboticallyactuated. As they are manually adjusted by sliding the square tubing within brackets and setting the position by tightening set screws, the precision and accuracy of adjustment depends upon the dexterity of the patient-side assistant performing these adjustments.

Likewise, the positioning of the lens within the bezel is done manually as was specified and recommended by the goals of the project. The on-site patient-side assistant selects the correct lens and places it in the bezel. The offset from the patient's eye is determined both by the gross adjustment of the base depth position as well as the offset of the lens in reference to the patient's eye. The placement of the lens within the bezel also is dependent upon the assistant. If a twist-lock clamp is implemented, the lens will self-center. However, the current prototype uses set screws to allow for lens size adjustments and the centering of the lens is dependent upon both the lens diameter as well as how well the assistant positions the lens within the bezel.

The partial rotations of the lens in both the horizontal and vertical directions were actuated, however. These were tested to obtain specifications similarly to the slit-lamp. These are summarized in Table 8-3 below.

Table 8-3: Lens Holder Partial Rotational Movement Summary (in degrees)

\begin{tabular}{|l|r|r|}
\hline & Average & Standard Deviation \\
\hline Horizontal Rotation & 2.2 & 0.42 \\
\hline Vertical Rotation & 2.3 & 0.48 \\
\hline
\end{tabular}


The table shows that the lens holder movements were very small and fairly consistent. This was necessary and expected given the small motors and the high precision required of this motion. The lens holder worked with much better precision and consistency than most of the slit-lamp actuators.

The lens rotation was not implemented in this phase of the project, so no data from the physical prototype could be obtained. It can be assumed that given that the same actuator, construction material and similar loads, the full rotation would perform approximately within the specifications of the partial rotations.

Discussion of these results will be provided in the next chapter as with the notes and observations from the robotic slit-lamp.

Performance of the actuated portion of the lens holder can be briefly summarized as:

- Vertical and horizontal partial rotational movement of the lens holder: $2.3^{\circ} \pm 0.5^{\circ}$

\subsection{Comparison of Prototype Performance to Traditional Use}

The comparison of the robotic specifications noted above to performance using traditional (in-person) control is provided in this section. These comparisons are not quantitatively made. This is because sufficient data was not found in a literature search and human testing was not performed as it was 
not within the scope of this project due to institutional, time and resource restrictions. However, some clear comparisons were made due to handling the base ophthalmic equipment before it was roboticized and also from observations made while gathering information about the project requirements when working with an ophthalmologist.

The original prototype was actually tested both manually and with the robotic attachments. The particular model of slit-lamp used for this project was old and very well worn. The tracks on the base sometimes caused the base to move awkwardly and the swinging of the arms or turning of the knobs did not always move smoothly nor in the desired increment. Due to these factors, it was actually fairly difficult to use, even by an ophthalmologist. It quickly became clear why this unit had been upgraded with a newer model by a previous owner. Still, it was a functional device despite the fact that it required more effort to move and manually get into alignment compared to newer models. This effort was in both in dexterity and time and it was very difficult to get good views of the points of interest on the practice eye.

In the robotic implementation, the slit-lamp still exhibited the same difficult behaviors. However, the motions were kept partially in check by the allowed increments and extra stability gained through the damping of the servo motor actuators that were attached to all moving joints. Either slit-lamp arm could not suddenly move further than desired when an area of high friction was overcome because the force of the servo automatically dampened the 
tendency to overshoot and moved the arm to the next incremental position. The same was true of the knobs, though these were much more stable as they had less inertia to keep them moving. The base of the slit-lamp did provide some trouble even for the robotic platform. The tracks and wheels had irregularities that made motions jerky and greatly upset the accuracy in one direction in particular. However, even these erratic motions were limited by the servos that dampened motions and held unactivated joints still.

The main advantage of the robotic implementation of the slit-lamp was that it was controlled from a computer and not manually. This meant that the user could simply click desired motions on the interface and the actuators would perform that movement. It was much more ergonomic than leaning over and looking into the binocular microscope eyepieces and moving all of the components of the slit-lamp by hand. Sitting upright and not having to constantly lean in at varying directions and degrees as the slit-lamp was moved proved to be helpful in enduring the time aspect of getting the slit-lamp targeted correctly. Also, when one motion was made, it was separated from any other motions being made at the same time. This meant that an adjustment in one direction by one actuator did not create an inadvertent adjustment in another direction or to another component. This created a condition that actually made the slit-lamp more cooperative than it had been. Overall, the robotization of the slit-lamp of the slit-lamp had a positive effect and it made the operation easier to use than by hand. 
The lens holder was another story. The first prototype consisted of two different iterations of lens holder designs. The first was a wire-driven serial arm. This was described in more detail in previous chapters. This design proved to be a failure for long term use. While it initially worked fairly well, the components developed wear very quickly and the holder soon would not hold up the lenses. The motions, while very accurate, did not have sufficient range to move the lens into all of the needed positions. It did suffice in preliminary testing of the physical prototype. A teleoperation test as well as several videos were obtained using this lens holder before complete failure.

The second lens holder was a simple micro-servo actuated pan-and-tilt mechanism that is commercially available and intended for small video cameras. Again, this designed is discussed in more details in previous chapters. This iteration proved to be much more stable than the first. It was also much more responsive and allowed for more than enough range of motion. Because it was a commercially-available device intended for another purpose, it did have some limitations. The remote center of rotation was not located in the correct place. The lens holder did not rotate around the eye, but instead at a point lower and further away from the eye than desired. This aspect made it more difficult to test, but the motions were small and could not be improved during any the testing that was done.

The third, and final in the scope of this work, iteration of the lens holder was the most robust and worked well. It allowed for gross adjustments in all three 
directions necessary via manual means. The lens holder portion was robotically-controlled and did rotate around the eye in both the vertical and horizontal directions. The implemented version did not have the lens rotation fully functioning, but this did not hinder any of the simulated examinations and testing that were performed. The feature can be added in the laser-ready version in a later prototype. This lens holder as implemented used the same micro-servos as in the pan-tilt mechanism of the second iteration. Due to this, the incremental motions were not as sharp as desired. However, they did work and allowed for adequate testing with proper rotation around the artificial eye. In this case, a human physician would almost certainly provide smaller motions, but would not be able to hold as steady for so long as shown in other work [52]. The lens holder also provides for greater comfort and better ergonomics as the physician does not need to lean over and keep an arm propped on the table in an attempt to hold the lens steady. More extensive testing with human subjects would be needed to determine how this device compares with traditional methods over long periods of time. It is likely in this scenario based on testing done that the lens holder performs better than a human hand. 


\section{DISCUSSION}

This work developed several prototypes. Controllers were developed to try to improve the real-life performance of the robotic systems. These had their successes and failures. This chapter will focus on exploring how well these controllers worked as well as their shortcomings.

\subsection{Prototype Comparisons to Prior Technologies}

It is difficult to fully compare OphthBot with exact prior technologies as comparable data on the device motion was never released on the robotic slitlamp developed in Thailand [21]. Instead, they focus on trials with human patients, which was not possible in the scope and limitations of this project. It is not evident that any other robotic slit-lamp was every physically developed nor that the system in Thailand underwent further development and testing.

Still, based on use of the slit-lamp and subjective comparison with the data taken from the device in Thailand, it is likely that OphthBot did not perform as well as the previously published device. Though not a large difference, it can be estimated that the ophthalmologists would find the Thailand robot to be more sensitive than the device presented in this work. This is to be expected as based on appearance, the previous robot used a new slit-lamp with more expensive components and was also controlled on-site rather than remotely. 
This eliminated many problems and allowed for quicker and sharper responses. However, it is clear that the Thailand robotic slit-lamp took nearly three times as long for the ophthalmologists to use than the standard slit-lamp. In this case, based on limited testing on OphthBot before and after being made robotic, it would seem that both prototypes performed similarly or that perhaps OphthBot was even a little easier to use compared to its non-robotic version. It is hoped that future testing will provide quantitative results to be able to make more certain comparisons on a later date.

With regards to the therapy robots, very little exists in quantitative results of previous technology. As with the ophthalmic robot, no tests nor studies with human clients were used with the systems developed here due to the difficulties such trials entail. Therefore, quantitative comparisons were not possible. Prior robotic humanoid systems were observed and the systems developed here performed in similar manners and with similar capabilities as those.

\subsection{Improvements of OphthBot 2 over OphthBot 1}

The differences between the two ophthalmic prototypes were not as large as originally intended. Still, some improvements were made and more was learned so that project continues to improve. 
The use of gears rather than friction-fit discs for the microscope and lamp arms is one of the major changes in the second iteration of the prototype. It offered the promise of more consistent performance and the reduction, if not elimination, of slippage. In practice, however, there was not much pace in which to attach the servo brackets. As plastic was used, the brackets tended to bend and allowed the servos to lift away from the slit-lamp so that the gears no longer meshed. A firmer bracket structure would solve this problem.

The friction drives of the knobs were replaced with belt drives that worked by friction as well. While this was an improvement in that it made adjustments much easier, the belts used were friction tape and it required more frequent adjustment and replacement than the rubber discs. It is hard to tell whether any costs or time are saved long term due to this change. Based on the more stable performance of the belt systems in both prototypes, at least while the belts are in good working order, it is likely that using more permanent rubber or silicone belts as used in OphthBot 1 combined with the OphthBot 2 mechanisms would offer the best of both designs.

The main improvement between the systems was the change of a wheeled base platform to an $X-Y$ table. The $X-Y$ table performed markedly better than the wheeled base. The results were a more controlled and consistent movement. It also offered movements that were more minute and thus superior to that offered by the micro adjustment joystick on the slit-lamp. Of all the changes and improvements in OphthBot 2, this is certainly the most 
important. Based on the information that was published, it not only out performed OphthBot 1 in these motions, but the Thailand prototype as well. It was determined that the $\mathrm{X}-\mathrm{Y}$ table, or similar design, is the mechanism of choice for controlling the base motions of any robotic slit-lamp system.

\subsection{Controller Performance}

The initial controller performed well enough for the system to operate as intended. Though not perfect, it did complete the proof-of-concept. It performed well in many areas, but was lacking in others as can be seen from data shown in the previous chapter.

One of the biggest issues was that the ophthalmic project did not show any benefits from improved controllers nor more sophisticated control software. There are a number of possible reasons for why this was the case. The first is that the servos used had a limited resolution and no controller could overcome the physical limitations of these components. Still, the servos did not perform to the specifications provided by the manufacturer. While it is possible that the manufacturing quality was low and the servos acquired and tested were not capable of performing to optimal specifications, it is also possible that they require adjustments or particular types of commands in order to achieve their ideal performance. Very little information was provided by the manufacturer, even after directly making contact. The manufacturer was slow to respond and 
never completely divulged some information commonly known about other servos that would have cleared up uncertainty.

Another reason for a lack of performance improvement may be due to the relatively short motions performed. Though the movement sizes were adjusted in order to see if differences resulted in the better controller performances, the movements were never made to be extremely large nor fast. As noted before, large or quick motions are usually undesirable in manual use of a slit-lamp and are more so when used remotely. The robot was never tested at full speed or range of motion as it was deemed undesirable, even if the performance improved. The controller needed to improve the performance with smaller and slower motions or it was unsuitable for these applications.

It was certainly a disappointment to not be able to test a myriad of controller algorithms and conclusively determine which were superior than all others for these applications or for the hardware utilized. Still, something was learned from the attempt. While it is possible that other algorithms may show results, it is suggested by the research presented here that different hardware, at least different actuators, must be selected in order for this method to bear fruit. New control hardware is coming to market at all times and there constantly remains the necessity to evaluate and test the limits of the new offerings to get the most out of the current technology and advance the products and systems.

From work with the robotic arm project, it is clear that there are already other control solutions now available in addition to the system chosen as the 
main focus during this project. The EZ-B that was used in a second iteration for the arm controller design was compact, all-inclusive, and much easier to program than the original controller. It also costs approximately the same as the microcontroller and servo-driver combination that it replaced, so cost was not a hindrance in selecting that controller over the first. The use of this controller, or a similar integrated unit, on the other projects in this work would be an interesting next step for further research.

Auto-targeting of structures within the eye is a new goal of the controller design. It was not completed due to limitations of the actuator resolutions and because it was not part of the stated scope of this work. However, the groundwork was provided through the kinematics of the slit-lamp provided in Chapter 7. A simplified slit-lamp with only the necessary components and actuators placed in more ideal locations is the next logical step for the prototype. In some respects, this is no longer the familiar slit-lamp of physicians, but from the remote control point of view, it will be identical. The patient will certainly see the difference, but a compact and efficient design should only be less intimidating and more accepted. This compact design will allow for better control no matter what hardware and software is selected. It will allow for more focus to be put on improving the programming and functionality of the system as it moves into clinical trials and commercialization. 


\subsection{Experimental Errors and Uncertainty}

The controller sometimes didn't work as well as desired in the OphthBot prototypes, as noted previously. This was caused by many factors. Some of the possible and known causes of issues, uncertainty, and errors are discussed here. These were introduced in both the models and in the actual implemented hardware, so both aspects will be mentioned.

\subsubsection{Model Factors}

Error in control systems can be introduced from multiple sources. One of the main sources is the model. Depending upon the methodology used, the design of a controller and the success of implementation is heavily dependent upon the accuracy of the model. If the actual system differs too far from the model, then it allows unexpected or undesired results.

One major factor that was not accurate in the models of the ophthalmic system was that of friction. It was included in some instances when model data was taken from measuring forces and torques in the system, so it was not completely omitted as with many system models. However, as with nearly all complex models, it could not be completely and realistically included both due to simplifications of the task and for hardware reasons that are discussed in further details in the next section.

As noted before, inertial movements of components that may move in the actual system were not modeled. The binocular and light arms are the main 
factors here, though all parts have the potential to move and have an effect. These were neglected because the slow movement of the system did not allow for notable inertial effects. In addition, all components ideally would be locked into their position by actuators that control their position, even in the case where movements may cause inertial disturbances.

Ultimately, future iterations will result in the model being less of a source of error. As the amount and size of hardware is reduced to just the necessary components and movements, the simplified equations representing the physical prototype will perfectly match the specialized essential hardware components. Full electronic control of features that can be implemented without hardware components will be made furthering the reduction in complexity.

\subsubsection{Hardware Factors}

Sources of error and uncertainty came into play with the hardware and software that was used for implementation of the OphthBot prototypes.

As with the model, friction appears again in this section. Uneven friction is one of the main sources of error. Friction in the actual system, even if it were to be modeled exactly for one instant, changed frequently during use. After extended manual use of the slit-lamp, inconsistencies and changes in friction over time were noticed. These changes were not always stiffer, as would be the case in the deterioration of lubrication, but sometimes components became 
more free-moving. They would also sometimes go back to stiffer motions again or vice versa. Due to the age of the vintage slit-lamp, there was wear of nearly all of the components and uneven lubrication noted in the device; It was impossible to fully plan for and overcome all the friction issues without full replacement of the base slit-lamp. These issues will be encountered if retrofit kits are commercialized for older systems, so it will remain a challenge in need of better solutions in which to overcome. Stronger and more accurate actuators, in addition to more robust control systems, can be developed in future iterations so that more consistent rotation motions are made despite these friction factors.

Another significant factor were the tracks on which the base of the slit-lamp slid. These tracks remained in use for OphthBot 1, but were not necessary and were omitted in OphthBot 2. The tracks sometimes caused issues because they were no longer completely flat and had protrusions that caused the slitlamp to sometimes stick in one position or move more than expected after an incremental movement command. This can be most clearly seen in the measurements of the base motions shown in Table 8-1 as well as Figure 8-2. The erratic behavior was due almost entirely to the slit-lamp becoming stuck on the tracks or moving quickly due to depressions. While some of this was due to friction, most was due to some warping of the metal over the years. Not only were they no longer completely flat, but they were also no longer completely parallel as the spacing between the rails were not even. Finally, the guides on 
top of the rails were bent. While this was repaired upon receipt of the slit-lamp, the foundation of the rail surfaces had warped enough to continue to cause issues and it attempts to fully straighten them did not hold. All of these conditions made it such that the travel of the wheels in the tracks were more difficult for some spots so that motion would be hindered and then begin again abruptly. As can be noted by the data shown in Chapter 8, removal of the track system proved to eliminate much of the hardware factors in control variability and allowed for a much more stable and robust system. 


\section{CONCLUSION}

This work produced two iterations of a prototype for a robotic slit-lamp. It also contributed research into using a similar controller in other areas such as pediatric rehabilitation. The controller was not improved by applying control theory techniques, but the prototypes still proved to be successes. They completed their goals of being proof-of-concepts for these technologies and introduced technical innovations.

\subsection{Implications of Research}

The prototypes developed in this work have the potential to increase options of care for people all over the world. These are specifically shown for the fields of ophthalmology and rehabilitation.

Teleophthalmology allows individuals in remote areas with limited access to physicians to receive expert care via tele-examinations and telesurgery. Soldiers and civilians in war zones will be able to quickly receive ophthalmic care on-site without risking the lives of specialists who will otherwise not be willing or available to provide the care. Emergency rooms and clinics will be able to offer near-immediate access to ophthalmologists even when one is not physically on-site. Patients in areas with access to specialists will be able to benefit from getting second opinions from experts located other regions who may be more knowledgeable about rare or complicated conditions. In turn, this 
can help ophthalmologists work together and share knowledge and expertise among themselves easier, faster, and more directly than ever before.

This work specifically pointed out the example of pediatric rehabilitation where inexpensive, but easily adaptable and robust control systems, are a great benefit. Prototypes were developed to show the possibility of creating affordable and fully customized humanoid systems to interact with patients, whether in the same room or in a remote location. These also allow for modeling of behaviors and tasks to children with different abilities in a way that typically-capable adult therapists are rarely able to perform fully and realistically.

The innovations in this dissertation can influence technologies in many other industries as well. Since this work uses hardware commonly used by those influenced by the Maker Movement, it is hoped that this work will help propel increased research interest in this area. Increasing numbers of professional engineers will hopefully embrace the concept of performing design in areas commonly thought of as only for hobbyists. Further collaboration and research will help to increase the creative and technical accomplishments of Makers all over the world as well as inspire talented hobbyists to pursue professional research and engineering themselves. 


\subsection{Future Directions and Recommendations}

This work, as with all academic endeavors, is never complete. There is plenty of room for other engineers to pick up and further this research both directly and in branching fields of study.

One area that allows for direct continuation of work is in the improvement of the remote ophthalmology prototype. This work leaves the prototype ready to be implemented in a laser-integrated slit-lamp and tested with both examination and surgical capabilities present. Many areas of improvement still exist in the mechanical design of actuation methods of the slit-lamp as well as the lens holder. Work can also be done designing a system from the ground up to be a robotic slit-lamp. Unlike this work where an existing slit-lamp was adapted, making a slit-lamp specifically to be used remotely will likely lead to many improvements in the motion and ease of operation of the system. This observation is based on preliminary studies performed during this work.

Work can be continued and is planned by the author in applying principles of this work toward pediatric rehabilitation systems. Child therapy devices offer challenges in accommodating a wide range of sizes and abilities. Developing safe control strategies, both active and passive, is critical. Children do grow to be adults, so this work can naturally be applied to adult therapeutic needs as well. It is not to exclude the possibility of applying this technology to adults, but many more researchers prefer to concentrate on adult patients than pediatric situations due to the extra complexities of the later. 
Finally, more work can be done in developing and implementing advanced control techniques for use in popular low-cost hardware that exists and will perpetually be developed on placed in the market. This work would appear to have no end in sight. While there are companies making new hardware for doit-yourself enthusiasts, there has not been as much academic input as compared with the industrial counterparts. Though it may not lead to patents or fame, this area is exploding in popularity with the general public and many people would benefit from having increased control options that work in their creations. It can only make everything better if school children, teachers and hobbyists are helping to develop and advance technology alongside engineers and scientists. 


\section{REFERENCES}

1. Afram, A. and Janabi-Sharifi, F. (2014) "Theory and applications of HVAC control systems - A review of model predictive control (MPC)," Building and Environment, 72, pp. 343-355.

2. Alessio, A. and Bemporad, A. (2009) "A survey on explicit model predictive control," Nonlinear Model Predictive Control, pp. 345-369, Springer Berlin Heidelberg.

3. Artstein, Z. (1980) "Discrete and continuous bang-bang and facial spaces or: look for the extreme points," SIAM Review, 22(2), pp. 172185.

4. Bekerman, I., Gottlieb, P. and Vaiman, M. (2014) "Variations in eyeball diameters of the healthy adults," Journal of Ophthalmology, Article ID 503645.

5. Bennett, C. and Sabanovic, S. (2013) "Perceptions of affective expression in a minimalist robotic face," Human Robot Interaction Proceedings, Tokyo, Japan, pp. 81-82.

6. Blumenkranz, M.S., Yellachich, D., Andersen, D.E., Wiltberger, M.W., Mordaunt, D., Marcellino, G.R., and Palanker, D. (2006) "Semiautomated patterned scanning laser for retinal photocoagulation," Retina, 26(3), pp. 370-376.

7. Bohren, J., Paxton, C., Howarth, R., Hager, G.D. and Whitcomb, L.L. (2016) "Semi-autonomous telerobotic assembly over high-latency networks," 11th ACM/IEEE International Conference on Human Robot Interaction, pp. 149-156.

8. Borggraefe, I., Schaefer, J.S., Klaiber, M., Dabrowski, E., AmmannReiffer, C., Knecht, B., Berweck, S., Heinen, F. and Meyer-Heim, A. (2010) "Robotic-assisted treadmill therapy improves walking and standing performance in children and adolescents with cerebral palsy," Official Journal of the European Paediatric Neurology Society, 14, pp. 496-502. 
9. Bourges, J-L., Hubschman, J-P., Burt, B., Culjat, M. and Schwartz, S.D. (2009) "Robotic microsurgery: corneal transplantation," British Journal of Ophthalmology, 93, pp. 1672-1675. DOI:10.1136/bjo.2009.157594.

10. Bourges, J-L., Hubschman, J-P., Wilson, J., Prince, S., Tsao, T-C., and Schwartz, S. (2011) "Assessment of a Hexapod Surgical System for Robotic Micro-Macro Manipulations in Ocular Surgery," Ophthalmic Research, 46, pp. 25-30.

11. Britton, M. and Andersson, A. (2000) "Home rehabilitation after stroke: Reviewing the scientific evidence on effects and costs," International Journal of Technology Assessment in Health Care, 18(3), pp. 842-848.

12. Buccella, C., Cecati, C., and Latafat, H. (2012) "Digital control of power converters - A survey," IEEE Transactions on Industrial Informatics, 8(3), pp. 437-447.

13. Bullies, K. (2005) "Robot rehab," Technology Review, 108(9), pp. 2930.

14. Burdea, G.C., Cioi, D., Kale, A., James, W.E., Ross, S.A. and Engsberg, J.R. (2013) "Robotics and gaming to improve ankle strength, motor control, and function in children with Cerebral Palsy A case study series," IEEE Transactions on Neural Systems and Rehabilitation Engineering, 21(2), pp. 165-173.

15. Burgar, C.G., Lum, P.S., Shor, P.C. and Van der Loos, H.F.M. (2000) "Development of robots for rehabilitation therapy: The Palo Alto VA/Stanford experience," Journal of Rehabilitation Research and Development, 37(5), pp. 663-673.

16. Buso, S., Fasolo, S., Malesani, L., and Mattavelli, P. (2000) "A deadbeat adaptive hysteresis current control," IEEE Transactions on Industry Applications, 36(4), pp. 1174-1180.

17. Camarillo, D.B., Krummel, T.M., and Salisbury, J.K. (2004) "Robotic technology in surgery: past, present, and future," The American Journal of Surgery, 188(4), pp. 2-15. 
18. Camden, C., Tetreault, S., Swaine, B. (2010) "Rehabilitation for Children - How is it Different from Rehabilitation for Adults?" International Encyclopedia of Rehabilitation, Article 274.

19. Campion, G., Bastin, G., and Dandrea-Novel, B. (1996) "Structural properties and classification of kinematic and dynamic models of wheeled mobile robots," IEEE transactions on robotics and automation, 12(1), pp. 47-62.

20. Carpi, F. and De Rossi, D. (2007) "Bioinspired actuation of the eyeballs of an android robotic face: concept and preliminary investigations," Bioinspiration \& Biommetics, 2, pp. S50-S63.

21. Charukamnoetkanok, P., Ekkachai, K., Klanarongran, N., Leelasawassuk, T., Komeswarakul, P., Suramethakul, P., Thonginnetra, O., Asawaphureekorn, S., Sintuwong, S., Tungpimolrut, K., Kongprawechon W., Pangputhipong, P. (2009, August) "Robotic slit-lamp for tele-ophthalmology," ICROS-SICE International Joint Conference, pp. 2535-2539. Fukuoka, Japan.

22. Childress, D.S. (2002) "Development of rehabilitation engineering over the years: As I see it," Journal of Rehabilitation Research and Development, 39(6), pp. 1-10.

23. Clement, R.G.E., Bugler, K.E., and Oliver, C.W. (2011) "Bionic prosthetic hands: a review of present technology and future aspirations," The Surgeon, 9, pp. 336-340.

24. Cong, M., Wu, Y., Liu, D., Du, Y., Wen, H., and Yu, J. (2011) "Simulation of 6-DOF parallel robot for coupling compensation method," Information Technology Journal, 10, pp. 428-433.

25. Cortes, P., Kazmierkowski, M.P., Kennel, R.M., Quevedo, D.E., and Rodríguez, J. (2008) "Predictive control in power electronics and drives," IEEE Transactions on Industrial Electronics, 55(12), pp. 4312-4324.

26. Dijikers, M.P., deBear, P.C., Erlandson, R.F., Kristy, K. and Geer, D.M. (1991) "Patient and staff acceptance of robotic technology in occupational therapy: A pilot study," Journal of Rehabilitation Research and Development, 28(2), pp. 33-44. 
27. Dougherty, D. (2012) "The maker movement," Innovations, 7(3), pp. 11-14.

28. Dunst, C.J., Bruder, M.B., Trivette, C.M., and Hamby, D.W. (2006) "Everyday activity settings, natural learning environments, and early intervention practices," Journal of Policy and Practice in Intellectual Disabilities, 3(1), pp. 2-10.

29. Ereso, A.Q., Garcia, P., Tseng, E., Gauger, G., Kim, H., Dua, M.M., Victorino, G.P., and Guy, T. S. (2010) "Live transference of surgical subspecialty skills using telerobotic proctoring to remote general surgeons," Journal of the American College of Surgeons, 211(3), pp. 400-411.

30. Fasoli, S.E., Fragala-Pinkham, M., Hughes, R., Hogan, N., Krebs, H.I., and Stein, J. (2008) "Upper limb robotic therapy for children with hemiplegia," American Journal of Physical Medicine and Rehabilitation, 87(11), pp. 929-9.36.

31. Fehlings, D., Switzer, L., Findlay, B., and Knights, S. (2013) "Interactive computer play as 'motor therapy' for individuals with cerebral palsy," Seminars in Pediatric Neurology, pp. 127-138.

32. Fine, H.F., Wei, W., Goldman, R.E., and Simaan, N. (2010) "Robotassisted ophthalmic surgery," Canadian Journal of Ophthalmology, 45(6), pp. 581-584.

33. Fischer, K., Kirstein, F., Jensen, L.C., Krueger, N., Kuklinski, N., aus der Wieschen, M.V. and Savarimuthu, T.R. (2016) "A comparison of types of robot control for programming by demonstration," 11th ACM/IEEE International Conference on Human Robot Interaction, pp. 213-220.

34. Fuller, T.A. (1980) "The Physics of Surgical Lasers," Lasers in Surgery and Medicine, 1, pp. 5-14.

35. Gama Melo, E.N., Aviles Sanchez, O.F., and Amaya Hurtado, D. (2014) "Anthropormorphic robotic hands: a review," Ingenieria $y$ Desarrollo, 32(2), pp. 279-313. 
36. Grace, K.W., Colgate, J.E., Glucksberg, M.R. and Chun, J.H. (1993) "A six degree of freedom micromanipulator for ophthalmic surgery," Proceedings of the IEEE Conference on Robotics and Automation, pp. 630-635. Atlanta, Georgia.

37. Grace, K.W., Jensen, P.S., Colgate, J.E., and Glucksberg, M.R. (1998) "Teleoperation for ophthalmic surgery: From the Eye Robot to feature extracting force feedback," Automedica, 16, pp. 293-310. New York.

38. Guerrouad, A., and Vidal, P. (1989) "SMOS: stereotaxical microtelemanipulator for ocular surgery," Proceeding of the Annual International Conference of the IEEE in Engineering in Medicine and Biology Society, 3, pp. 879-880. Seattle, Washington. DOI: 10.1109/IEMBS.1989.96028.

39. Gupta, P.K., Jensen, P.S., and de Juan, E. (1999) "Surgical forces and tactile perception during retinal microsurgery," Medical Image Computing and Computer-Assisted Intervention (MICCAl'99), LNCS 1679, pp. 1218-1225.

40. Hagag, B., Abovitz, R., Kang, H., Schmitz, B., and Conditt, M. (2011) "RIO: Robotic-Arm Interactive Orthopedic System MAKOplasty: User interactive haptic orthopedic robotics," J. Rosen, B. Hannaford, R.M. Satava (Eds.), Surgical Robotics - Systems, Applications, and Visions. Springer.

41. Hannaford, B., Rosen, J., Friedman, D.W., King, H., Roan, P., Cheng, L., Glozman, D., Ma, J., Kosari, S.N., and White, L. (2013) "Raven-II: an open platform for surgical robotics research," IEEE Transactions on Biomedical Engineering, 60(4), pp. 954-959.

42. Hendrix, R. (2011) Robotically assisted eye surgery: A haptic master console, Dissertation, Technische Universiteit Eindhoven.

43. Hills, J.W. and Jensen, J.F. (1998) "Telepresence technology in medicine: principles and applications," Proceedings of the IEEE, 86(3), pp. 569-580.

44. Hogan, N. (1985) "Impedance control: An approach to manipulation: Part I - Theory," Transactions of the ASME Journal of Dynamic Systems, Measurement, and Control, 107, pp. 1-7. 
45. Hogan, N. (1985) "Impedance control: An approach to manipulation: Part II - Implementation," Transactions of the ASME Journal of Dynamic Systems, Measurement, and Control, 107, pp. 8-16.

46. Hogan, N. (1985) "Impedance control: An approach to manipulation: Part III - Applications," Transactions of the ASME Journal of Dynamic Systems, Measurement, and Control, 107, pp. 17-24.

47. Hogan, N. and Krebs, H.I. (2011) "Physically interactive robotic technology for neuromotor rehabilitation," Progress in Brain Research, 192, pp. 59-68.

48. Hubschman, J-P., Bourges, J-L., W Choi, W., Mozayan, A., Tsirbas, A., Kim, C-J., and Schwartz, S-D. (2010) "The Microhand': a new concept of micro-forceps for ocular robotic surgery," Eye, 24, pp. 364-367.

49. Interactive Motion Technologies (2013) InMotion Robot-Assisted Therapy: Evidence-Based Neurorehabilitation, Pamphlet; Watertown, Massachusetts.

50. Jung, J.J., Gallego-Pinazo, R., Lleo-Perez, A., Huz, J.I., and Barbazetto, I.A. (2013) "NAVILAS laser system focal laser treatment for diabetic macular edema - one year results fo a case series," The Open Ophthalmology Journal, 7, pp. 48-53.

51. Jung, L.A., McCormick, K.M., and Jolivette, K. (2004) "Early intervention in rural natural environments: making the most of your time," Rural Special Education Quarterly, 23(3), pp. 30-35.

52. Kaba, K., Baksh, F., Almashan, Y., Alsubaei, B., Bach, A., Morris, M. and Tosunoglu, S. (2017) "Minature Robotic Arm to Manipulate Opthalmic Lenses," Proceedings of the $30^{\text {th }}$ Florida Conference on Recent Advances in Robotics, May 12, Boca Raton, Florida.

53. Kazanzides, P., Chen, Z., Deguet, A., Fischer, G.S., Taylor, R.H., and DiMaio, S.P. (2014) "An Open-Source Research Kit for the da Vinci Surgical System," Proceedings of the 2014 IEEE International Conference on Robotics \& Automation, pp. 6434-6439, Hong Kong, China. 
54. Kaur, S. (2012) "How medical robotics are going to affect our lives: Pushing frontiers with the first lady of emerging technologies," IETE Technical Review, 29(3), pg. 184-187.

55. Kernt, M., Ulbig, M., Kampik, A. and Neubauer, A.S. (2013) "Navigierte Netzhautlasertherapie," Ophthalmologe, 110, pp. 776782. [In German]

56. Krebs, H.I., Brashers-Krug, T., Rauch, S.L., Savage, C.R., Hogan, N., Rubin, R.H., Fischman, A.J. and Alpert, N.M. (1998) "Robot-aided functional imaging: Application to a motor learning study," Human Brain Mapping, 6, pp. 59-72.

57. Krebs, H.I., Hogan, N., Aisen, M.L. and Volpe, B.T. (1998) "Robotaided neurorehabilitation," IEEE Transactions on Rehabilitation Engineering, 6(1), pp. 75-87.

58. Krebs, H.I., Volpe, B.T., Aisen, M.L. and Hogan, N. (2000) "Increasing productivity and quality of care: Robot-aided neurorehabilitation," Journal of Rehabilitation Research and Development, 37(6), pp. 639-652.

59. Krebs, H.I., Volpe, B.T., Aisen, M.L., Hening, W., Adamovich, S., Poizner, H., Subrahmanyan, K., and Hogan, N. (2003) "Robotic applications in neuromotor rehabilitation," Robotica, 21(1), pp. 3-11.

60. Kulshrestha, M., Kelly, S., and Mahmood, U. (2011) "Teleophthalmology in practice," G. Graschew (Ed.), Telemedicine Techniques and Applications, pp. 393-412. Croatia, InTech. DOI: $10.5772 / 18175$

61. Kwoh, Y.S., Hou, J. ; Jonckheere, E.A. ; Hayati, S. (1988) "A robot with improved absolute positioning accuracy for CT guided stereotactic brain surgery ," IEEE Transactions on Biomedical Engineering, 35(2), pp. 153-161.

62. Lanfranco, A.R., Castellanos, A.E., Desai, J.P., and Meyers, W.C. (2004) "Robotic surgery: a current perspective," Annals of surgery, 239(1), pp. 14-21. 
63. Lapeyre, M., Rouanet, P., Grizou, J., Nguyen, S., Depraetre, F., Le Falher, A., and Oudeyer, P-Y. (2014) "Poppy Project: open-source fabrication of 3D printed humanoid robot for science, education and art," Digital Intelligence, Nantes, France, p. 6.

64. Larsson, D., Irving, D., Effendi, S., Dubin, J., Bach, A., Morris, M. and Tosunoglu, S. (2017) "Ophthalmic Robot," Proceedings of the $30^{\text {th }}$ Florida Conference on Recent Advances in Robotics, May 12, Boca Raton, Florida.

65. Lum, M.J., Friedman, D.C., Sankaranarayanan, G., King, H., Fodero, K., Leuschke, R., Hannaford, B., Rosen, J. and Sinanan, M.N. (2009) "The RAVEN: Design and validation of a telesurgery system," The International Journal of Robotics Research, 28(9), pp. 1183-1197.

66. Maliki, R., Alhaidar, D., Attallah, K., Alsalem, S., Morris, M., and Tosunoglu, S. (2017) "Robotic Hand That Teaches Sign Languages," Proceedings of the $30^{\text {th }}$ Florida Conference on Recent Advances in Robotics, May 12, Boca Raton, Florida.

67. Mann, R.W. (2002) "Engineering design education and rehabilitation engineering," Journal of Rehabilitation Research and Development, 39(6), pp. 23-38.

68. Mardihn, C.Y., Tornow, R.P., and Kruse, F.E. (2010) "Lasers in Ophthalmology," Physics Procedia, 5, pp. 631-636.

69. Marescaus, J., Leroy, J., Rubino, F., et. al. (2002) "Transcontinental robot-assisted remote telesurgery: feasibility and potential applications," Annals of Surgery, 235, pp. 487-492.

70. Marino, H., Bergeles, C., and Nelson, B.J. (2014) "Robust electromagnetic control of microrobots under force and localization uncertainties," IEEE Transactions on Automation Science and Engineering, 11(1), pp. 310-316.

71. Mariotti, S.P. (2012) Global Data on Visual Impairments 2010, World Health Organization, WHO/NMH/PBD/1201, Geneva, Switzerland.

72. Mattei, T.A., Rodriguez, A.H., Sambhara, D., and Mendel, E. (2014) "Current state-of-the-art and future perspectives of robotic technology in neurosurgery," Neurosurgical review, 37(3), pp. 357366. 
73. Mellmann, H. and Cotugno, G. (2011) "Dynamic motion control: adaptive bimanual grasping for a humanoid robot," Fundamenta Informaticae, 112(1), pp. 89-101.

74. Mettler, L., Ibrahim, M., and Jonat, W. (1998) "One year of experience working with the aid of a robotic assistant (the voicecontrolled optic holder AESOP) in gynaecological endoscopic surgery," Human Reproduction, 13(10), pp. 2748-2750.

75. Meyer-Heim, A., Borggraefe, I., Ammann-Reiffer, C., Berweck, S., Sennhauser, F.H., Colombo, G., Knecht, B., and Heinen, F. (2007) "Feasibility of robotic-assisted locomotor training in children with central gait impairment," Developmental Medicine and Child Neurology, 49, pp. 900-906.

76. Meyer-Heim, A. and van Hedel, H.J.A. (2013) "Children with cerebral palsy: current state and clinical implementation," Seminars in Pediatric Neurology, 20, pp. 139-145.

77. Michelman, P. (1998) "Precision object manipulation with a multifingered robot hand," IEEE Transactions on Robotics and Automation, 14(1), pp. 105-109.

78. Mitchell, B., Koo, J., lordachita, I., Kazanzides, P., Kapoor, A., Handa, J., Hager, G., and Taylor, R. (2007) "Development and application of a new steady-hand manipulator for retinal surgery," Proceedings of the IEEE International Conference on Robotics and Automation, pp. 623-629. Rome, Italy.

79. Mordatch, I., Lowrey, K. and Todorov, E. (2015) "Ensemble-ClO: fullbody dynamic motion planning that transfers to physical humanoids," 2015 IEEE/RSJ International Conference on Intelligent Robots and Systems, pp. 5307-5314.

80. Morris, M. (2007) A Planar Cable-Driven Robotic Device for Physical Therapy Assistance, M.S. Thesis, Florida Atlantic University, Boca Raton, Florida.

81. Morris, M. and Masory, O. (2008) "A novel cable-driven robot for rehabilitation," $2^{\text {nd }}$ Israeli Conference on Robotics, November 19, Herzlia, Israel. 
82. Muir, P.F. and Neuman, C.P. (1987) "Kinematic modeling of wheeled mobile robots," Journal of Field Robotics, 4(2), pp. 281-340.

83. Nakano, T., Sugita, N., Ueta, T., Tamaki, Y., and Mitsuishi, M. (2009) "A parallel robot to assist vitreoretinal surgery," International Journal of Computer Assisted Radiology and Surgery, 4, pp. 517-526.

84. Oliveira, H.P., Sousa, A.J., Moreira, A.P., and Costa, P.J. (2009) "Modeling and assessing of omni-directional robots with three and four wheels," Contemporary Robotics: Challenges and Solutions, pp. 207-230.

85. Olazo, R., Soles, G., Mendoza, A., Drada, J-C., Tosunoglu, S., and Morris, M. (2017) "The Humanoid Rehabilitation Project," Proceedings of the 30th Florida Conference on Recent Advances in Robotics, May 12, Boca Raton, Florida.

86. O'Sullivan, S., Schmitz, T. and Fulk, G. (2014) Physical Rehabilitation, 6th Edition, F. A. Davis Company, Philadelpha, Pennsylvania, p. 409.

87. Pitcher, J.D., Wilson, J.T., Tsao, T-C., Schwartz, S.D., and Hubschman, J-P. (2012) "Robotic eye surgery: past, present, and future," Journal of Computer Science Systems Biology, S3. OMICS Publishing Group. DOI: 10.4172/jcsb.S3-001.

88. Pititeeraphab, Y. and Sangworasil, M. (2015) "Design and construction of system to control the movement of the robot arm," Proceedings of the 2015 Biomedical Engineering International Conference, IEEE.

89. Power, M. (2013) "Confessions of a drone warrior," GQ Magazine, November.

90. Qin, S.J. and Badgwell, T.A. (2003) "A survey of industrial model predictive control technology," Control Engineering Practice, 11(7), pp. 733-764.

91. Qiu, Q., Ramirez, D.A., Saleh, S., Fluet, G.G., Parikh, H.D., Kelly, D. and Adamovich, S.V. (2009) "The New Jersey Institute of Technology Robot-Assisted Virtual Rehabilitation (NJIT-RAVR) system for children with cerebral palsy: a feasibility study," Journal of NeuroEngineering and Rehabilitation, 6(40). 
92. Rahimy, E., Wilson, J., Tsao, T-C., Schwartz, S., and Hubschman, JP. (2013) "Robot-assisted intraocular surgery: Development of the IRISS and feasibility studies in an animal model," Eye, 27, 972-978.

93. Ramos, L., Valencia, S., Verma, S., Zornoza, K., Morris, M., and Tosunoglu, S. (2017) "Robotic Face to Simulate Humans Undergoing Eye Surgery, "Proceedings of the 30th Florida Conference on Recent Advances in Robotics, May 12, Boca Raton, Florida.

94. Rawlings, A.L., Woodland, J.H., and Crawford, D.L. (2006). "Telerobotic surgery for right and sigmoid colectomies: 30 consecutive cases," Surgical Endoscopy And Other Interventional Techniques, 20(11), pp. 1713-1718.

95. Reinkensmeyer, D.J., Schmit, B.D. and Rymer W.Z. (1999) "Mechatronic assessment of arm impairment after chronic brain injury," Technology and Health Care, 7, pp. 431-435.

96. Reinkensmeyer, D.J., Emken, J.L. and Cramer, S.C. (2004) "Robotics, motor learning, and neurologic recovery," Annual Review of Biomedical Engineering, 6, pp. 497-525.

97. Riviere, C.N., Rader, R.S., and Khosla, P.K. (1997). "Characteristics of hand motion of eye surgeons," Proceedings of the 19th Annual International Conference of the IEEE Engineering in Medicine and Biology Society, 4, pp. 1690-1693.

98. Riviere, C.N., Rader, R.S., and Thakor, N.V. (1998) "Adaptive canceling of physiological tremor for improved precision in microsurgery," IEEE Transactions on Biomedical Engineering, 45(7), pp. 839-846.

99. Rosen, J. (2013) "Surgical Robots," M. Culjat, R. Singh, and H. Lee (Eds.), Medical Devices: Surgical and Image Guided Technologies. John Wiley \& Sons.

100. Salvador, M.J., Silver, S., and Mahoor, M.H. (2015) "An emotion recognition comparative study of autistic and typically-developing children using the Zeno robot," Proceedings of the 2015 IEEE International Conference on Robotics and Automation, Seattle, Washington, pp. 6128-6133. 
101. Scassellati, B., Admoni, H., and Mataric, M. (2012) "Robots for Use in Autism Research," Annual Review of Biomedical Engineering, 14, pp. 275-294.

102. Shaw, C., Bourkiza, R., Wickham, L., Mccarthy, I., and Mckechnie, C. (2017) "Mechanical exposure of ophthalmic surgeons: a quantitative ergonomic evaluation of indirect ophthalmoscopy and slit-lamp biomicroscopy," Canadian Journal of Ophthalmology/Journal Canadien d'Ophtalmologie, to appear.

103. Shoham, M., Lieberman, I.H., Benzel, E.C., Togawa, D., Zehavi, E., Zilberstein, B., Roffman, M., Bruskin, A., Fridlander, A., Joskowicz, L., Brink-Danan, S., and Knoller, N. (2007) "Robotic assisted spinal surgery - from concept to clinical practice," Computer Aided Surgery, 12(2), pp. 105-115.

104. Sluijs, E.M., Kok, G.J., and van der Zee, J. (1993) "Correlates of exercise compliance in physical therapy," Physical Therapy, 73(11), pp. 783-786.

105. Smania, N., Bonetti, P., Gandolfi, M., Cosentino, A., Waldner, A., Hesse, S., Werner, C., Bisoffi, G., Geroin, C., and Munari, D. (2011) "Improved gait after repetitive locomotor training in children with cerebral palsy," American Journal of Physical Medicine and Rehabilitation, 90(2), pp. 137-149.

106. Sommer, A., Taylor, H.R., Ravilla, T.D., West, S., Lietman, T.M., Keenan, J.D., Chiang, M.F., Robin, A.L. and Mills, R.P. (2014) "Challenges of ophthalmic care in the developing world," JAMA Ophthalmology, 132(5), pp. 640-644.

107. Stokoe, W.C. (2005). "Sign language structure: An outline of the visual communication systems of the American deaf," The Journal of Deaf Studies and Deaf Education, 10(1), pp. 3-37.

108. Sun, Y. and Sundar, S.S. (2016) "Psychological importance of human agency: how self-assembly affects user experience of robots," 11th ACM/IEEE International Conference on Human Robot Interaction, pp. 189-196. 
109. Sun, Z., Balicki, M., Kang, J., Handa, J., Taylor, R., and lordachita, I. (2009) "Development and preliminary data of novel integrated optical micro-force sensing tools for retinal microsurgery, "Proceedings of the IEEE International Conference on Robotics and Automation, pp. 1897-1902. Kobe, Japan.

110. Ting, C-H., Yeo, W-H., King, Y-J., Chuah, Y-D., Lee, J-V., and Khaw, W-B. (2014) "Humanoid robot: a review of the architecture, applications and future trend," Research Journal of Applied Sciences, Engineering and Technology, 7(7), pp. 1364-1369.

111. Tsirbas, A., Mango, C., and Dutson, E. (2007) "Robotic ocular surgery," British Journal of Ophthalmology, 91, pp. 18-21. DOI: 10.1136/bjo.2006.096040.

112. Ueta, T., Yamaguchi,Y., Shirakawa, Y., Nakano, T., Ideta, R., Noda, Y., Morita, A., Mochizuki, R., Sugita, N., Mitsuishi, M., and Tamak Y. (2009) "Robot-assisted vitreoretinal surgery: Development of a prototype and feasibility studies in an animal model," Ophthalmology, 116, pp. 1538-1543.

113. Uneri, A., Balicki, M.A., Handa, J., Gehlbach, P., Taylor, R.H., and lordachita, I. (2010) "New steady-hand eye robot with micro-force sensing for vitreoretinal surgery," Proceedings of the 3rd IEEE RAS \& EMBS International Conference on Biomedical Robotics and Biomechatronics, pp. 814-819. Tokyo, Japan.

114. Vossmerbaeumer, U. (2010) "Application principles of excimer lasers in ophthalmology," Medical Laser Application, 25, pp. 250-257.

115. Wagner, B. [2001] Eye Examination with the Slit Lamp, Publication Number 000000-1152-355, Carl Zeiss Meditec AG, Germany.

116. Walsh, F. (2016) "Robot operates inside eye in world first," $B B C$ News, September 9, Health.

117. Wei, W., Goldman, R., Simaan, N., Fine, H., and Chang, S. (2007) "Design and theoretical evaluation of micro-surgical manipulators for orbital manipulation and intraocular dexterity," Proceedings of the IEEE International Conference on Robotics and Automation, pp. 3389-3395. Rome, Italy. 
118. Williams, R.L., Carter, B.E., Gallina, P., and Rosati, G. (2002) "Dynamic model with slip for wheeled omnidirectional robots," IEEE Transactions on Robotics and Automation, 18(3), pp. 285-293.

119. Wu, H., and $\mathrm{Li}, \mathrm{H}$. (2017) "Application of the da Vinci in thoracic surgery," AME Medical Journal, 2(2).

120. Xu, Z., Kumar, V. and Todorov, E. (2013) "A low-cost and modular, 20-DOF anthropomorphic robotic hand: design, actuation and modeling," 13th IEEE-RAS International Conference on Humanoid Robotics, pp. 368-375.

121. Yin, X. and Bowling, A. P. (2006) "Dynamic performance limitations due to yielding in cable-driven robotic manipulators," Journal of Mechanical Design, 128(1), pp. 311-318.

122. Yu, D-Y., Cringle, S.J., and Constable I.J. (1998) "Robotic ocular ultramicrosurgery," Australian and New Zealand Journal of Ophthalmology: Clinical and Epidemiology, 26, pp. S6-S8.

123. Zeilig, G., Weingarden, H., Zwecker, M., Dudkiewicz, I., Block, A. and Esquenazi, A. (2012) Journal of Spinal Cord Medicine, 35(2), pp. 96101.

124. Zuniga, J., Katsavelis, D., Peck, J., Stollberg, J., Petrykowski, M., Carson, A. and Fernandez, C. (2015) "Cyborg beast: a low-cost 3Dprinted prosthetic hand for children with upper-limb differences," BMC Research Notes, 8(1), p. 10. 


\section{VITA \\ MELISSA MORRIS}

Born in Michigan, USA

2001

$2000-2003$

Engineering Intern, Camera Operating Systems Sensormatic Electronics Corporation Boca Raton, Florida

B.S., Electrical Engineering, Cum Laude

Florida Atlantic University

Boca Raton, Florida

2004

Student Talon Award for Outstanding Leadership

Florida Atlantic University

Boca Raton, Florida

2005

Engineering Intern, Virtual Manufacturing

Ford Motor Company

Cologne, Germany

$2003-2007$

M.S., Mechanical Engineering

Graduate Certificate, Bioengineering

Florida Atlantic University

Boca Raton, Florida

$2008-2010$

Research Assistant, Robotics

Technion - Israel Institute of Technology

Haifa, Israel

$2011-2017$

Ph.D., Mechanical Engineering

Florida International University

Miami, Florida

\section{SELECTED PUBLICATIONS AND PRESENTATIONS}

Morris, M. and Tosunoglu, S. (2016) "Teleoperated ophthalmic examination robot," Proceedings of the $29^{\text {th }}$ Florida Conference on Recent Advances in Robotics, May 12-13, Miami, Florida, pp. 282-285. 
Morris, M. (2013) "Flexible Robotic Technology for Human-Robot Interaction," Proceedings of the 26th Florida Conference on Recent Advances in Robotics, May 16-17, Tallahassee, Florida.

Morris, M. and Tosunoglu, S. (2012) "Comparison of Rechargeable Battery Technologies," American Society of Mechanical Engineers Early Career Technical Conference and Journal, November 2-3, Atlanta, Georgia.

Morris, M. and Raviv, D. (2009) "A Methodical Method for Determining Research Areas in Heart Disease Based on the Eight Dimensional Methodology for Innovative Problem Solving," Proceedings of the 2009 American Society for Engineering Education Annual Conference \& Exposition, June 14-17, Austin, Texas.

Morris, M. and Shoham, M. (2009) "Applications and Theoretical Issues of Cable-Driven Robots," Proceedings of the 22nd Florida Conference on Recent Advances in Robotics, May 21-22, Jupiter, Florida.

Morris, M. and Masory, O. (2008) "A Novel Cable-Driven Robot for Rehabilitation," Second Israeli Conference on Robotics, November 1920, Herzlia, Israel.

Raviv, D., Morris, M., and Ginsberg, K. (2005) "On Teaching and Assessing Engineering Innovation," Proceedings of the 2005 American Society for Engineering Education Annual Conference \& Exposition, June 14, Portland, Oregon.

Kelly, T., Baillargeon, P., Morris, M., and Morris, J. (2005) "Pipetting Application of a Pick and Place Robot," 18th Florida Conference on Recent Advances in Robotics, May 5-6, Gainesville, Florida.

Morris, M. (2004) "A Study of Bilateral Force Reflection Manipulators," $17^{\text {th }}$ Florida Conference on the Recent Advances in Robotics, May 6, Orlando, Florida.

Morris, M. (2004) "Portable Braille Computer Interface," Proceedings of the ASME Southeastern Region XI 2004 Regional Technical Conference and Journal, April 2-3, Mobile, Alabama. 